University of South Florida

DIGITAL COMMONS

Digital Commons @ University of

@ UNIVERSITY OF SOUTH FLORIDA

South Florida

USF Tampa Graduate Theses and Dissertations

USF Graduate Theses and Dissertations

January 2013

\title{
A Biocompatible SiC RF Antenna for In-vivo Sensing Applications
}

Shamima Afroz

University of South Florida, safroz@mail.usf.edu

Follow this and additional works at: https://digitalcommons.usf.edu/etd

Part of the Biomedical Engineering and Bioengineering Commons, and the Electrical and Computer Engineering Commons

\section{Scholar Commons Citation}

Afroz, Shamima, "A Biocompatible SiC RF Antenna for In-vivo Sensing Applications" (2013). USF Tampa Graduate Theses and Dissertations.

https://digitalcommons.usf.edu/etd/4428

This Dissertation is brought to you for free and open access by the USF Graduate Theses and Dissertations at Digital Commons @ University of South Florida. It has been accepted for inclusion in USF Tampa Graduate Theses and Dissertations by an authorized administrator of Digital Commons @ University of South Florida. For more information, please contact digitalcommons@usf.edu. 
A Biocompatible SiC RF Antenna for In-vivo Sensing Applications

by

\author{
Shamima Afroz \\ A dissertation submitted in partial fulfillment \\ of the requirements for the degree of \\ Doctor of Philosophy \\ Department of Electrical Engineering \\ College of Engineering \\ University of South Florida \\ Co-Major Professor: Sylvia W. Thomas, Ph.D. \\ Co-Major Professor: Stephen E. Saddow, Ph.D. \\ Gokhan Mumcu, Ph.D. \\ Robert Frisina, Ph.D. \\ Lennox Hoyte, M.D.
}

Date of Approval:

February 27, 2013

Keywords: silicon carbide, implantable, glucose sensor, biocompatability, biofunctionalization

Copyright $\odot$ 2013, Shamima Afroz 


\section{ACKNOWLEDGMENT}

I would like to express my deepest gratitude to my advisors, Dr. Sylvia W. Thomas and Dr. Stephen E. Saddow, for their help and guidance. I sincerely thank them for their encouragement, technical guidance and for giving me this research opportunity. This dissertation would not have been possible without their support. I am enormously grateful to all my committee members Gokhan Mumcu, Lennox Hoyte, Robert Frisina for their support and time. I specially thank Dr. Mumcu, who helped me with antenna design and simulations; he always made himself available whenever I needed his advice. I am grateful to Dr. Hoyte for the in vivo implantation of the devices. Sincere thanks to Dr. Karl Muffly for helping with tissue histological analysis. I appreciate Dr. Christopher Frewin for helping me with the preparation of blood mimicking liquids. Thanks to my collogues and friends Chris Locke, Alexandra Oliveros, Joe Registrar, Brandon Richard, Samuel Perez, Henry Cabra, Justin King, and Maysam Nezafati who offered help and support during this research. I want to thank all NREC members including Richard Everly and Robert Tufts for their assistance during this entire research. I would also like to thank USF Graduate School for the USF Graduate School Student Success (GSS) Fellowship and the National Science Foundation (NSF) E-STARS for their financial support during the endeavor.

I remember my father, who would be proud of me and has always been a source of my inspiration. I profoundly thank my mother, who is always there whenever I needed 
her. Very special thanks to my husband, Mehdi, for his endless moral support, encouragement and understanding from the very beginning! And last but not least to my loving son Shamiq, who was a little baby when I started and grew up a supportive toddler during this time- how do I articulate how understanding you were when it was mommy's study time. 


\section{TABLE OF CONTENTS}

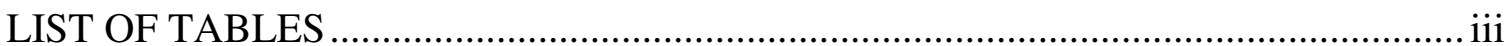

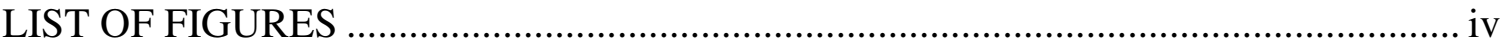

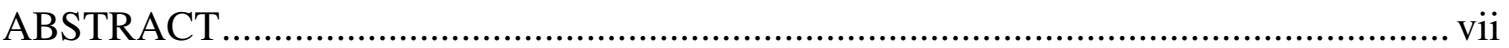

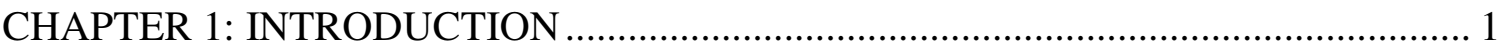

1.1 Research Objective and Motivation ................................................................. 1

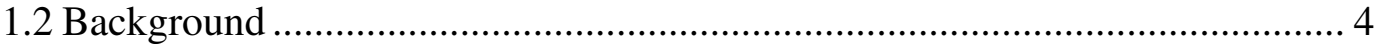

1.2.1 Existing Devices and Limitations ..................................................... 4

1.2.2 Mechanism for Glucose Monitoring Using RF Antenna ................... 7

1.2.3 Silicon Carbide as a Bio-compatible Material .................................. 9

1.3 Summary and Dissertation Organization ....................................................... 14

CHAPTER 2: ANTENNA DESIGN, GROWTH AND FABRICATION ........................ 16

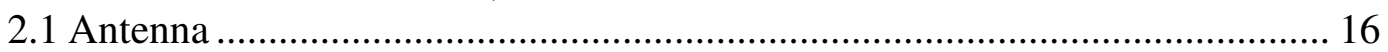

2.1.1 Theory of the Patch Antenna... ……………………...................... 19

2.1.2 Frequency Band for Medical Applications ........................................ 23

2.2 SiC Based Antenna Simulations and Design ............................................... 24

2.2.1 SiC Based Ti/Au Metal Antenna ……………………………...... 27

2.2.2 Different SiC Based Antenna Designs and Methodologies ............. 29

2.3 Chemical Vapor Deposition (CVD) Growth ................................................ 32

2.3.1 Epi-growth of 4H-SiC on SI 4H-SiC, Approach (a) ......................... 36

2.3.2 Growth of Poly 3C-SiC on SI 4H-SiC, Approach (b)...................... 39

2.3.3 Fully Planar Ion Implantation, Approach (c) ……………………... 43

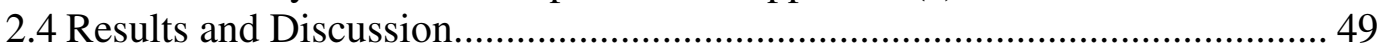

CHAPTER 3: SiC BASED ANTENNA AS A GLUCOSE BIOSENSOR ....................... 51

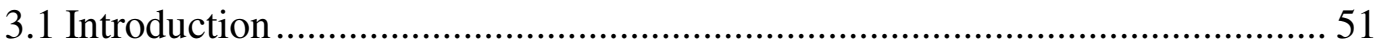

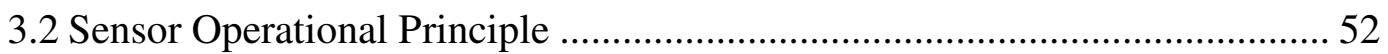

3.3 Antenna Imbedded in Animal Muscle and Fatty Tissue ................................ 54

3.4 Fat and Blood Plasma Mimicking Liquid Preparation...................................... 55

3.4.1 Mimicking Liquid's Permittivity ……………………………….... 56

3.4.2 Experimental Setup for Blood Glucose Monitoring …………….... 58

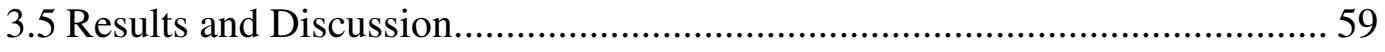

CHAPTER 4: IN VIVO INVESTIGATION OF ANTENNA MATERIALS .................... 64

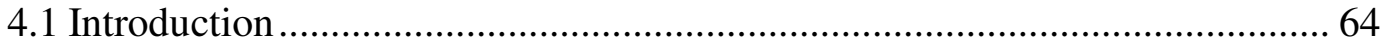




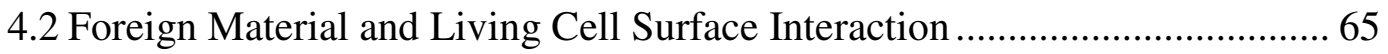

4.2.1 Protein Adsorption to Biologically Relevant Surfaces .................. 67

4.2.2 Surface Modification...................................................................... 69

4.2.3 Protein Repellent/Nonfouling Coating Material ........................... 69

4.2.4 Poly(ethyleneglycol) (PEG) Properties and Functionalization ........ 70

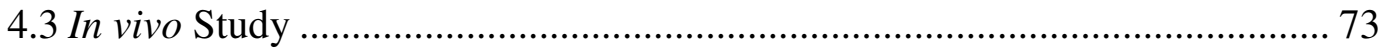

4.3.1 Materials and Methods ............................................................ 73

4.3.2 Sample Preparation on SiC-based Ti/Au Antenna......................... 75

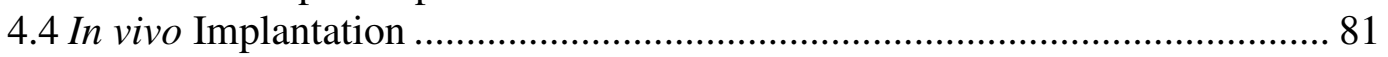

4.4.1 Implantation Procedure ............................................................ 81

4.4.2 Implants Removal and Tissue Collections ................................. 84

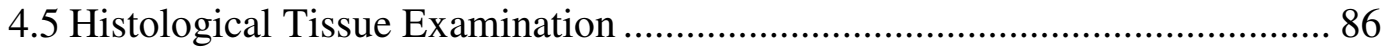

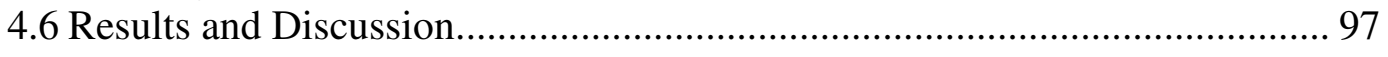

CHAPTER 5: SUMMARY AND FUTURE WORK .............................................. 99

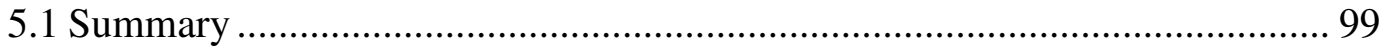

5.2 Recommendations for Future Works .................................................... 104

5.2.1 Antenna Design Optimization............................................... 105

5.2.2 Highly Doped Epilayer Optimization for all-SiC Antenna........... 106

5.2.3 Antenna Sensor Test in Pig Body with Operational Life-time ...... 107

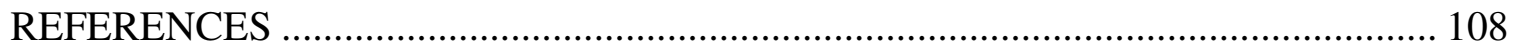

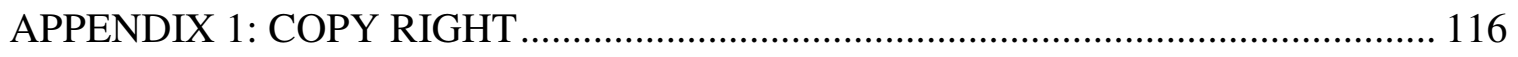

APPENDIX 2: IACUC PROTOCOL AND APPLICATION …................................ 117

APPENDIX 3: MICROTOMY TISSUE SECTIONING ....................................... 130

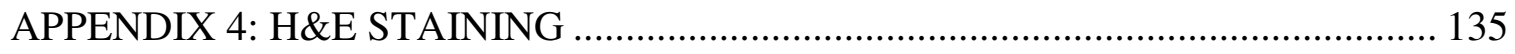




\section{LIST OF TABLES}

Table 1: Electrical, thermal and physical properties of different SiC ........................... 10

Table 2: Recipe for blood plasma mimicking liquid ..............................................56

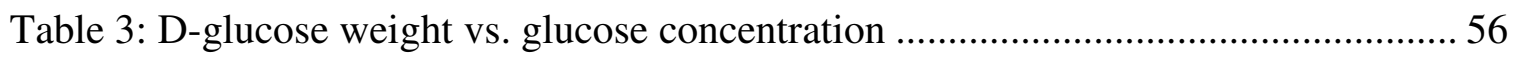

Table 4: Details of in vivo experimental implants in pig model................................. 75 


\section{LIST OF FIGURES}

Figure 1: Implanted patch antenna and the measurement of antenna sensor

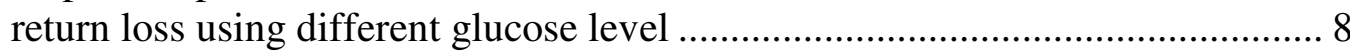

Figure 2: Fluorescent optical micrographs (a), (c), (d): B16, BJ and HaCaT cell morphology on $6 \mathrm{H}-\mathrm{SiC}$ after 72 hours

Figure 3: Flourescent micrographs of $\mathrm{Si}, 3 \mathrm{C}-, 4 \mathrm{H}-$, and $6 \mathrm{H}-\mathrm{SiC}$ showing platelet adhesion and aggregation

Figure 4: (a) Microstrip patch antenna, (b) electric field distribution (side view) 20

Figure 5: (a) Schematic of the $\mathrm{SiC}$ based antenna sensor (b) simulated radiation pattern

Figure 6: (a) All $\mathrm{SiC}$ antenna return loss; (b) dependence of radiation efficiency on doping level and substrate thickness

Figure 7: (a) Fabrication process; and (b) photograph of the Ti/Au patch on semiinsulating $4 \mathrm{H}-\mathrm{SiC}$ antenna

Figure 8: Simulation and experimental return loss of Ti/Au antenna............................... 29

Figure 9: Simulation of antenna response for $1 \times 10^{19}$ to $1 \times 10^{20} \mathrm{~cm}^{-3}$ doping concentration

Figure 10: Cross section of three envisioned approaches to realize fully biocompatible glucose sensor using $\mathrm{SiC}$ antenna.

Figure 11: Photograph of the MF2 CVD horizontal reactor at USF 35

Figure 12: Measured doping concentration increased with Silicon/Carbon (Si/C) ratio; the growth condition of the best film is also shown .

Figure 13: CVD growth process schedule used for approach (b)

Figure 14: AFM morphology of doped poly $3 \mathrm{C}-\mathrm{SiC}$ (a) $5 \times 5 \mathrm{~m}^{2}$ with a surface roughness of $16 \mathrm{~nm}$ RMS

Figure 15: Fabrication process flow of approach $\mathrm{b}$ 
Figure 16: The simulated and measured poly 3C-SiC antenna (approach (b)) responses showing the return loss and resonant frequency

Figure 17: Fabrication process flow of approach (c)

Figure 18: (a) The simulation for ion implantation on SiC for different energy levels to get high concentration of doping profile 44

Figure 19: (a) AFM micrographs of ion implanted surface morphology 46

Figure 20: (a) AFM micrographs of surface morphology after post ion implant annealing.....

Figure 21: All SiC antenna responses (structure (c)) compared with the $\mathrm{Ti} / \mathrm{Au}$ antenna response. 48

Figure 22: (a) Photograph of the experimental setup using a VNA, (b) SiC based $\mathrm{Ti} / \mathrm{Au}$ antenna resonance frequency shift vs. permittivity changes from fat/muscle (chicken and cow)

Figure 23: Measured relative permittivity of fat and different glucose levels of blood mimicking liquid vs. frequency

Figure 24: Setup for measuring the blood plasma glucose level (a) schematic diagram and; (b) photograph of the experiment 58

Figure 25: Measured and simulated (inset) $S_{11}$ response of the antenna as a function of glucose concentration within the blood mimicking solution

Figure 26: Measured $S_{11}$ response of the antenna to pig blood as a function of glucose concentration for (a) $67 \mathrm{mg} / \mathrm{dl}$, (b) $120 \mathrm{mg} / \mathrm{dl}$, (c) $270 \mathrm{mg} / \mathrm{dl}$, (d) $490 \mathrm{mg} / \mathrm{d}$, respectively

Figure 27: Measured relative resonance frequency shift dependence on glucose concentration.

Figure 28: The foreign body reaction illustration by higher organisms to an implanted synthetic material/biomaterial.....

Figure 29: Molecular structure of poly ethylene glycol (PEG) 71

Figure 30: Seven different antenna surfaces and samples (a) Ti/Au antenna on

$\mathrm{SI} 4 \mathrm{H}-\mathrm{SiC}$ as the base 74

Figure 31: Cross-section view of the $\mathrm{SiC}$ based $\mathrm{Ti} / \mathrm{Au}$ antenna 76 
Figure 32: Cross-section view of the $\mathrm{SiO}_{2}$ coated $\mathrm{SiC}$ based $\mathrm{Ti} / \mathrm{Au}$ antenna.

Figure 33: Cross-section of the PEG coated $\mathrm{SiC}$ based $\mathrm{Ti} / \mathrm{Au}$ antenna .......................... 78

Figure 34: (a) Cross-section of the a-SiC coated $\mathrm{SiC}$ based $\mathrm{Ti} / \mathrm{Au}$ antenna ..................... 79

Figure 35: Cross-section of the Poly 3C-SiC antenna showing all of the materials used to realize the antenna.

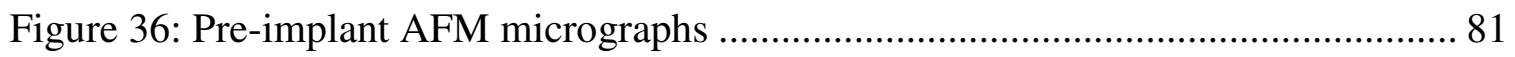

Figure 37: (a) Photograph of the subcutaneous implantation of the antenna sensor in pig....

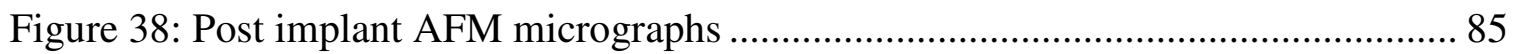

Figure 39: Histological tissue analysis of healthy tissue without any implant showing no signs of inflammation (original magnification $\mathrm{x} 40$ ).

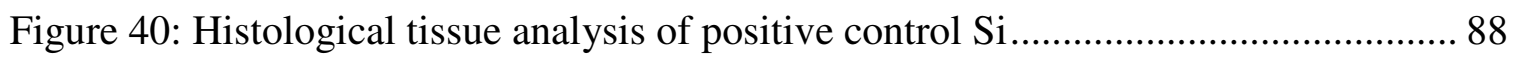

Figure 41: Photograph of the fibrous capsule after removal Si implant (30 days in vivo implantation specimen)

Figure 42: Histological tissue analysis of the bare $\mathrm{Ti} / \mathrm{Au}$ antenna ................................. 90

Figure 43: Histological tissue analysis of the all-SiC antenna .................................. 91

Figure 44: Histological tissue analysis of $\mathrm{SiO}_{2}$ coated $\mathrm{SiC}$-based $\mathrm{Ti} / \mathrm{Au}$ antenna............ 92

Figure 45: Histological tissue analysis of a-SiC coated $\mathrm{Ti} / \mathrm{Au}$ antenna.......................... 94

Figure 46: Histological tissue analysis of 3C- SiC/a-SiC coated sample ....................... 94

Figure 47: Histological tissue analysis of poly ethylene glycol (PEG)

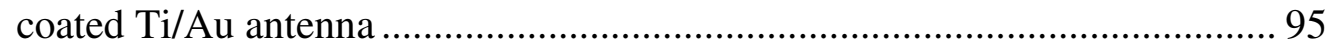

Figure 48: Seven different implant's histological tissue response.............................. 96 


\begin{abstract}
A continuous glucose sensor employing radio frequency (RF) signals is presented using the biocompatible material Silicon Carbide ( $\mathrm{SiC}$ ). Unlike biosensors that require direct contact with interstitial fluids to trigger chemical reactions to operate, this biocompatible $\mathrm{SiC}$ sensor does not require a direct interface. The sensing mechanism for this $\mathrm{SiC}$ sensor is based upon a shift in resonant frequency, as a function of change in glucose levels, which electrically manifests itself as a change in blood permittivity and conductivity. For in vivo applications the antenna sensor needs to operate inside the body environment, and it has been found that the best operational location of this biocompatible $\mathrm{SiC}$ sensor is within fatty tissue in close proximity to blood vessels. To test glucose levels, measurements using synthetic body fluid (SBF), which is electrically equivalent to blood plasma, were performed. Changes in sensor performance to varying glucose levels were measured and a shift in resonant frequency to lower values observed with increasing glucose level. In vitro sensor performance demonstrated that the sensor showed a dose dependent response to glucose concentration from $120 \mathrm{mg} / \mathrm{dl}$ to 530 $\mathrm{mg} / \mathrm{dl}$. A shift of $40 \mathrm{MHz}$ was observed corresponding to a $97 \mathrm{kHz}$ shift per $1 \mathrm{mg} / \mathrm{dl}$ change in blood glucose. Similarly the blood glucose levels were measured in pig blood using the same SiC based antenna sensor. The dependence of glucose concentration on resonance frequency observed with pig blood followed the same trend as the blood-
\end{abstract}


mimicking experiment discussed earlier. The sensor performance was linear with the frequency shift being a direct function of glucose concentration.

An in vivo experiment for foreign body response to subcutaneously-implanted antenna has been conducted using a pig/swine animal model. Tissue histology analysis showed that all-SiC antenna and poly ethylene glycol (PEG) coated Ti/Au antenna did not have any inflammatory immune response for 30 days. However, some inflammatory signs were found on bare Ti/Au antenna. The histological tissue analysis on $a$-SiC coated and single crystal 3C-SiC samples did not show any significant inflammatory response. 


\section{CHAPTER 1}

\section{INTRODUCTION}

\subsection{Research Objective and Motivation}

Implantable medical devices are at the center of much academic and technical research in biomedical engineering, medicine and biology. Much of the work surrounding implantable technology has been a global initiative. With the advancement of MEMS structures, numerous devices are being designed and fabricated using silicon ( $\mathrm{Si}$ ) as the primary material. This is logical since $\mathrm{Si}$ is a very well-known and reliable material, has excellent mechanical and electronic properties, and has been used in many sensing applications. While $\mathrm{Si}$ will continue to be the material of choice for short-term biomedical applications, considerable work is needed to select a biocompatible device material suitable for long term implantation that is, at the same time, capable of efficient sensing. The increased demand of biomedical devices to solve medical problems has motivated the search in vivo antennas that would permit both interoperable communications between sensors as well as data transfer into and out of the human body. In addition antenna sensors have been demonstrated but there is a clear need to realize these devices using fully biocompatible materials that do not cause an adverse tissue reaction nor endanger the life of a patient [1]. 
In recent years, considerable progress has been made in developing implantable biosensors that can continually monitor different health care issues, such as the glucose levels of a diabetic patient. The major problem that affects the lives of patients with a permanent disease, such as chronic diabetics, is obtaining daily blood samples multiple times to monitor their glucose levels. Such intermittent testing is laborious, often painful, and requires significant diligence to adequately monitor true glycemic patterns. The development of implantable biosensors has paved the road for developing continuous glucose monitoring systems [2-5]. Despite the intense research on implantable biosensors, present day biosensors cannot operate in-vivo for more than 10 days before issues of biofouling impact the sensing capabilities. This dissertation research was undertaken to overcome this issue by using a small sized implantable antenna that can be used for continuous glucose monitoring and sensing. The central hypothesis of this research is that an implantable RF antenna fabricated with biocompatible materials is well suited for long-term in-vivo systems. A particular example would be a reliable continuous glucose monitoring system able to control hypoglycemia, thus lessening the complications associated with the life threatening disease, diabetes. This is a key to improving the quality of the lives of patients living with this terrible disease.

Many biomedical devices require materials that are both biocompatible and have sensing capability. Biochemical sensors, biologically interfaced neural networks, and smart biomedical implants necessitate semiconductor materials, so that sensing can be performed using not only mechanical means, but also through electrical means as well (i.e., MEMS). To date, the biocompatibility of only a few crystalline semiconductors has been investigated, with $\mathrm{Si}$ and titanium dioxide $\left(\mathrm{TiO}_{2}\right)$ drawing most of the attention. 
However, Si has been shown to display different degrees of cytotoxicity, mostly due to its instability in aqueous solutions with subsequent formation of silica and silicates, which are known for their harmful effects on cells. On the other hand, $\mathrm{TiO}_{2}$, which can become a semiconductor upon ion implantation, does not display sufficiently satisfying electronic properties that may justify its adoption for sensing applications. Silicon carbide (SiC) is a semiconductor that is chemically inert to acids, alkalis, and salts, does not expand in liquid environments, and can be processed with the existing methods developed for the silicon ( $\mathrm{Si})$ industry [6,7]. It has also been found that $\mathrm{SiC}$ is a semiconducting material displaying biocompatibility, longevity, and great sensing potentiality, to fill the materials existing gap. Therefore this research utilizes $\mathrm{SiC}$ as the material of choice due to its great potential benefit as a robust biocompatible material for advanced implantable devices.

The scientific objective of this dissertation is the design of a fully implantable longterm continuous monitoring antenna using the biocompatible material SiC. This work is novel and can be distinguished by its innovation to utilize $\mathrm{SiC}$ as the device material, thus removing the need to encase biomedical devices with biocompatible materials and addresses the shorter lifetime issue of current sensors.

Once the overall scholarly objective of this work has been met, this technology could be transferred to various health care systems, not limited to glucose monitoring. This research effort will address a range of bioengineering questions: 1) Increased understanding of bio sensing/detecting and continuous diagnostic feedback; 2) Enhanced understanding of semiconductors as biomaterials and extended lifetime functionality using materials such as silicon carbide ( $\mathrm{SiC})$; and 3) System integration of implantable wireless devices using an RF antenna. 


\subsection{Background}

The search for a self-glucose monitoring solution started in the early 1970's. In 1971, Anton Clemens from USA filed the patent for a blood glucose monitor for point-of-care use in diabetic patients [8]. A glucose monitoring device called Ames Reflectance Meter which automatically assessed the enzyme-based reagent strips by changing their color. Prior to this, blood glucose had been estimated by reading the color change from a chart by eye from a chart The Ames Reflectance Meter was succeeded by the Ames Eyetone, but it was restricted to clinical areas such as hospital wards and physicians' offices. In 1978, the first portable devices for the self-monitoring of blood glucose became available. The self-blood glucose monitoring was acceptable to the patients; it also demonstrated that the technology improved glycaemic control. More iimportantly, selfmonitoring also reduced the frequency of hypoglycemia thus greatly extending patient quality of life.

\subsubsection{Existing Devices and Limitations}

For ideal glucose sensor, the predictable response to changing glucose concentrations should be fast. Under physiological conditions, it should have a long operational lifetime, but most of all it must be acceptable to the patient. Therefore, it would ideally provide real-time continuous information regarding glucose and should not require any user calibration. Continuous glucose monitoring provides real-time data to the patients and alarms the times of hypoglycemia or rapid glucose change by providing data about the direction, magnitude, duration, frequency and potential causes of fluctuations in blood glucose levels. Many technologies including non-invasive, minimally-invasive or 
invasive are being pursued to develop novel continuous glucose sensors and they may depend on interstitial fluid (ISF), transdermal technologies or sampling of blood.

Different non-invasive glucose sensors, such as optical transducers and different spectroscopic methods (infrared absorption spectroscopy; mid-infrared or near infrared, etc.) face multiple problems, including scattering of light, as well as specific absorption bands for accurate blood glucose sensing [8]. Another approach is to use techniques of impedance spectroscopy and electromagnetic coupling, which are based on impedance measurement of the dielectric parameters of blood [9-10]. This impedance spectroscopy / electromagnetic coupling technique has been used in different non-invasive glucose sensing applications where the resonant frequency, as well as the level of the return loss of the sensors, were shown to change with different glucose levels [11-14]. On the other hand, invasive glucose sensors such as subcutaneous needle-type sensors use enzyme electrodes. Enzymes are used in Enzyme electrodes which catalyze reduction-oxidation (redox) reactions; therefore in doing so electrons are accepted or donated. Hydrogen per oxide is generated from Glucose Oxidase $\left(\mathrm{GO}_{\mathrm{X}}\right)$ and platinum electrode is used to measure this peroxide amperometrically which is directly proportional to the glucose concentration. A subcutaneous needle-type amperometric enzyme electrode based on $\mathrm{GO}_{\mathrm{X}}$ is used by the Medronic (Medronic Diabetese, Northridge, California) for continuous glucose monitoring system (CGMS). It has a 4-min response time with an average of 63 hours recording time. A significant sensor drift requires four times calibration per day. This sensor drifting is postulated partly due to the alteration of local blood flow as a result of protein and cell coating of the sensor, variable tissue oxygen tension, and tissue interferents and wound response to the sensor. After explanting the 
sensor, the sensitivity remains low but after repeated washing it improves; therefore it is reversible $[15,16]$.

Some newer devices by the DexCom STS (Dexcom, San Diego, California) and Abbott FreeStyle Navigator (Abbott Diabetes, Alameda, California) also use a subcutaneous enzyme-based electrode. FreeStyle Navigator has a recording time of 5 days where the DexCom is a wireless system using low power radio and has a recoding time of 72 hours. Therefore, these sensors have the similar life time limitations.

Microdialysis systems which use a fine, hollow microdialysis fiber placed subcutaneously is another type of subcutaneous sensor is. Interstitial fluid (ISF) glucose freely diffuses into the fiber where the fiber is perfused with isotonic fluid from an exvivo reservoir. Since the sensor is ex-vivo; it avoids the problems associated with the fouling by protein and cells. This sensor has less drift than CGMS, but still it requires one calibration per day. To assess clinical performance, Microdialysis and subcutaneous needle type sensors have been compared to assess clinical performance - the microdialysis probe displayed greater accuracy with an additional lag of 7 minutes than the needle type sensor. Therefore, the microdialysis systems have inherent physical lag time for the dialysate to be pumped to the sensor which uses more power. The device is larger because they require perfusate solution, which makes this device very uncomfortable to the patients. On the other hand, the physical and chemical properties of the membrane may change over time, as will the tissue characteristics such as volume, temperature, hydration and pressure. In conclusion, for the continuous glucose monitoring, the sensors have several issues/limitations associated with their systems - the glucose sensor needs replacement frequently; the RF unit and other external devices are 
bulky adding discomfort for the patient already wearing an insulin pump; the RF unit is continuously powered requiring frequent battery replacement; The FDA still recommends a confirmation test using conventional blood sample from the finger; and most importantly the sensors have a long response time.

The key to the success of all implantable glucose sensors is the need to ensure reliable performance when exposed to living tissue and fluids. The term biofouling has gained common usage as a description of the buildup of proteins and fibroblasts that accompany the body's immune reaction to the sensor. The body attempts to ward off the foreign material by forming an encapsulate which is typically 100 microns or more thick. This layer is problematic for any device that seeks to sample serum or blood since it acts as a diffusion barrier, not to mention an electrically insulating layer. Encasing the implant with a biocompatible material is necessary to prevent the implant from harming the surrounding tissue and to reducing the effects of the capsular contracture [17]. A better proposed solution would be a sensor made from a fully biocompatible material for longer periods of functionality in the body and to overcome the current sensor life time limitations.

\subsubsection{Mechanism for Glucose Monitoring Using RF Antenna}

The central hypothesis of glucose monitoring using the RF antenna is that the changes in the medium surrounding the antenna [Figure1: Dr. Erdem Topsakal, Mississippi State University] affect antenna parameters such as input impedance and resonance frequency, and these changes can be used to estimate the patient's plasma glucose level. The changes of other minerals in the blood such as calcium, chloride, potassium, and 
magnesium will have minor effect in antenna parameters as compare to glucose levels.

Fortunately, this assumption is quite reasonable in reality thus further motivating this approach to developing a glucose sensor using an RF antenna.

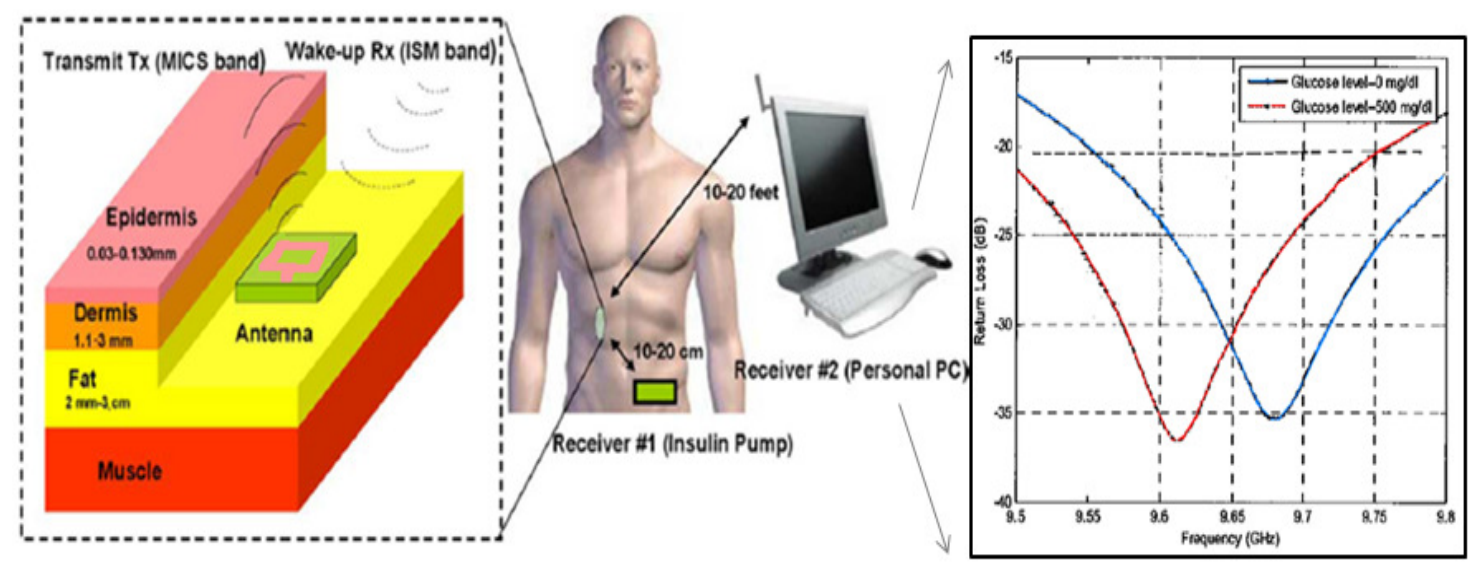

Figure 1: Implanted patch antenna and the measurement of antenna sensor return loss using different glucose level. The resonance frequency shifted according to the glucose levels. [Dr.Erdem Topsakal, Mississippi State University]

For instance, the glucose concentration of a diabetic patient's blood may vary between $30 \mathrm{mg} / \mathrm{dl}$ and $400 \mathrm{mg} / \mathrm{dl}$ while sodium and chloride levels, although they exist in large quantities; only vary from $310 \mathrm{mg} / \mathrm{dl}$ to $333 \mathrm{mg} / \mathrm{dl}$ and $337 \mathrm{mg} / \mathrm{dl}$ to $372 \mathrm{mg} / \mathrm{dl}$, respectively [University of Michigan Medical Library]. Other minerals exist in very low quantities in human blood. In addition, because the blood glucose concentration in the collected samples had gone through in-vitro manipulation throughout the study, the concentrations of other minerals in a specific sample remained unchanged during measurements. This approach guaranteed that the changes occurring in the antenna parameters are only due to glucose manipulation of the blood sample. Figure 1 shows the measured antenna sensor return loss for different glucose levels. The resonance frequency shifted due to the presence of different glucose levels thus motivation the research conducted here during this dissertation. 


\subsubsection{Silicon Carbide as a Bio-compatible Material}

Silicon carbide ( $\mathrm{SiC})$ is physically strong and chemically inert material, which hypothetically makes it an ideal for interaction with the harsh body environment. Amorphous $\mathrm{SiC}(\mathrm{a}-\mathrm{SiC})$ and polycrystalline $\mathrm{SiC}$ (poly-SiC), have been used as coating materials for many implantable biomedical devices. These materials have also been used for clinical studies - such as bone prosthetics and heart stents - which confirmed the biocompatibility of these forms of $\mathrm{SiC}$ [18-20]. a-SiC was also used as a thin film coating for the Utah Probe neural prosthetic constructed from silicon [21]. One of the advantages of these forms of $\mathrm{SiC}$ over single crystal $\mathrm{SiC}$ is that they can be deposited at lower temperatures, and they have mostly insulating properties because of the large amount of crystalline defects present in the material [18-21]. Like silicon, silicon carbide is a semiconducting material, which enables the creation of complex electrical devices upon doping incorporation. Many of the device processing techniques used in Si technology can be utilized in $\mathrm{SiC}$ processing [7]. Unlike many semiconductors, $\mathrm{SiC}$ may be found/synthesized in many crystal forms, known as polytypes, having approximately 170 different crystal observed organizations, but with a common stoichiometry (i.e., all forms contain bi-layers of $\mathrm{Si}$ and C) [7]. Some of the physical and electrical properties of some of the most common $\mathrm{SiC}$ polytypes are summarized alongside with $\mathrm{Si}$ in Table 1. 4H-SiC and $6 \mathrm{H}-\mathrm{SiC}$ - two of the hexagonal polytypes of $\mathrm{SiC}$ - have been commercially available as substrates and have also been fabricated into electronic devices [21-23]. These materials have shown good biocompatibility, non-toxicity, and low biofouling when used as electrode myocardial biosensors $[24,25]$. SiC doping incorporation must be done either during the growth process, which is known as in-situ doping, or through ion- 
implantation and subsequent activation; it has no ability to diffuse any dopants into the crystalline structure [7]. Dry etching methods like reactive ion etching or deep reactive ion etching can be used to etch $\mathrm{SiC}$ [7].

Table 1: Electrical, thermal and physical properties of different SiC [26-28]

\begin{tabular}{|c|c|c|c|c|}
\hline & 3C-SiC & $4 \mathrm{H}-\mathrm{SiC}$ & $6 \mathrm{H}-\mathrm{SiC}$ & $\mathrm{Si}$ \\
\hline Band Gap (eV) & 2.36 & 3.23 & 3.05 & 1.1 \\
\hline $\begin{array}{l}\text { Electron Mobility }\left(\mathrm{cm}_{2} \mathrm{~V}^{-1} \mathrm{~s}^{-1}\right) \\
\text { at } 300 \mathrm{~K}\end{array}$ & $<800$ & $<900$ & $<400$ & 1500 \\
\hline $\begin{array}{l}\text { Hole Mobility }\left(\mathrm{cm}_{2} \mathrm{~V}^{-1} \mathrm{~s}^{-1}\right) \\
\text { at } 300 \mathrm{~K}\end{array}$ & $<320$ & $<90$ & $<120$ & 450 \\
\hline Breakdown Field $\left(\mathrm{MV} \mathrm{cm}^{-1}\right)$ & 1 & $3-5$ & $3-5$ & 0.25 \\
\hline $\begin{array}{l}\text { Thermal expansion @300 K } \\
@ 1500-1600 \mathrm{~K}\left(10^{-6} \mathrm{~K}^{-1}\right)\end{array}$ & $\begin{array}{l}3.8 \\
5.5\end{array}$ & NA & $\begin{array}{l}4.3_{-} c \text { axis } \\
4.7_{\_} c \text { axis }\end{array}$ & $\begin{array}{l}2.6 \\
4.56-4.6\end{array}$ \\
\hline $\begin{array}{l}\text { Thermal conductivity } \\
(\mathrm{W} / \mathrm{cm}-\mathrm{K})\end{array}$ & 5 & 4.9 & 4.9 & 1.5 \\
\hline Young's Modulus (GPa) & \multicolumn{3}{|c|}{300 to 700} & 47 \\
\hline Elastic Modulus (GPa) & 433 & 424 & & 170 \\
\hline
\end{tabular}

Silicon carbide $(\mathrm{SiC})$ is one of the few semiconducting materials that combine biocompatibility and great sensing potentiality. SiC's chemical inertness, superior tribological properties, hydroxyapatite-like osseointegration, and well-known hemocompatibility make it a very promising candidate for in vitro biosensors, biologically interfaced neural networks and intelligent implantable medical devices [19, 
29, 30]. In order for this semiconductor to become a material of choice for biotechnological applications, its biocompatibility was compared to that of the leading crystalline semiconductors.

The three main polytypes of $\mathrm{SiC}$ are the cubic form, namely $3 \mathrm{C}-\mathrm{SiC}$, and two hexagonal forms, namely $4 \mathrm{H}-$ and $6 \mathrm{H}-\mathrm{SiC}$. Dr. C. Coletti of the $\mathrm{SiC}$ group at the University of South Florida researched the in vitro biocompatibility of $\mathrm{SiC}$ to three lines of cells: B16-F10 mouse melanoma cells, BJ human fibroblasts, and human keratinocytes cells $(\mathrm{HaCaT})$, which were cultured onto the surfaces of these main polytypes [7]. The experimental approach was to assess the material's biocompatibility through observation of adherent cell morphology and proliferation on the SiC surface [30]. The protocols developed also involved positive and negative reaction controls which were bare $\mathrm{Si}$ and cell treated polystyrene (PSt) culture plates, respectively. The last surface served as a benchmark. Quantitative and qualitative assessments were performed using MTT [3-(4, 5-dimethylthiazol-2-yl)-2,5-diphenyltetrazolium bromide] assays and fluorescent microscopy, respectively. Since the $\mathrm{SiC}$ and $\mathrm{Si}$ samples used in this work presented similar topographical characteristics, substrate topography can be excluded as a cause of the reported SiC superior biocompatibility. The MTT assay results reported in Figure 2 clearly show that $\mathrm{SiC}$, in all its principal phases, performed better than $\mathrm{Si}$ as a substrate for cell culture, for all mammalian cell lines studied [30]. No statistically significant differences were found among the cell proliferation. A similar cell morphology and adhesion quality was observed for all the SiC- polytypes [31]. The optical inspection results obtained using fluorescent microscopy support the MTT quantitative results and provide additional useful information regarding cell morphology and adhesion quality, 
reprinted with permission [Appendix A] [32]. In general, cells cultured on $\mathrm{SiC}$ substrates appeared to be flattened and morphologically similar to the cells on the negative reaction control [Figure 2].
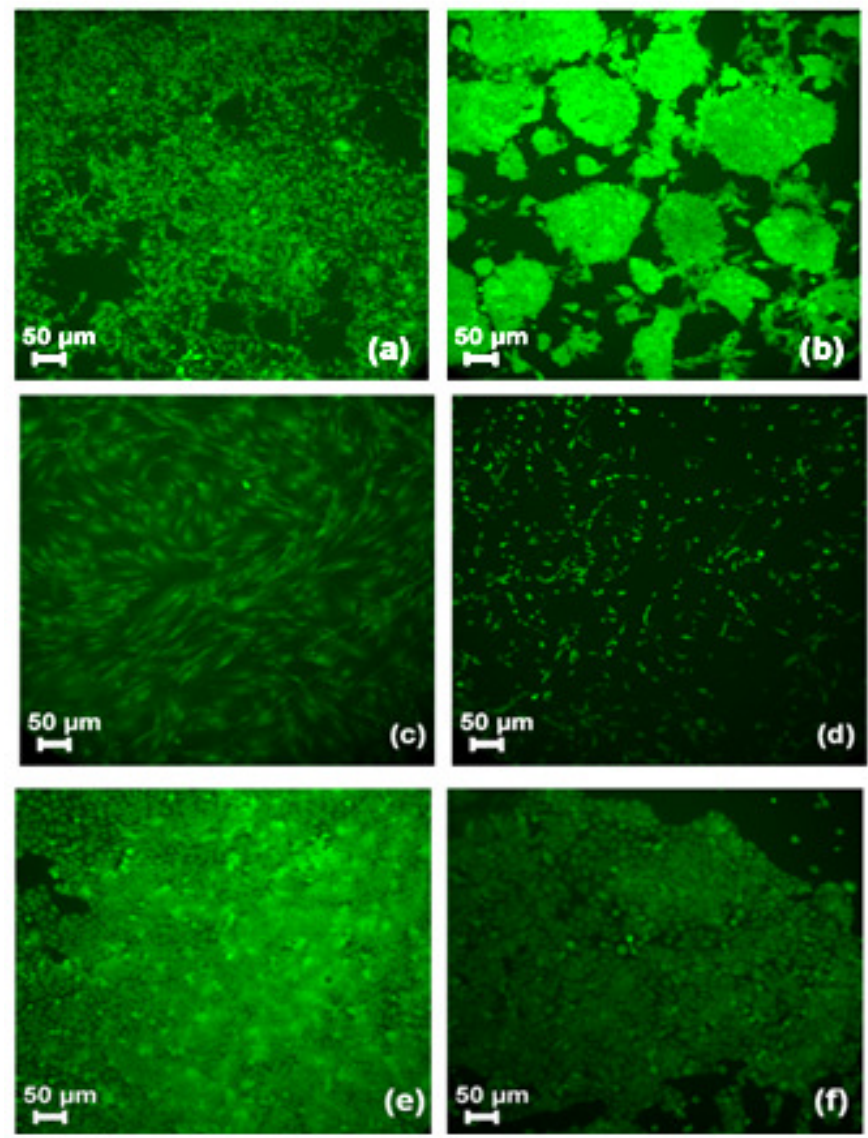

Figure 2: Fluorescent optical micrographs (a), (c), (d): B16, BJ and HaCaT cell morphology on $6 \mathrm{H}-\mathrm{SiC}$ after 72 hours. (b), (e), (f): B16, BJ and HaCaT cell morphology on Si after 72 hours [32]

To confirm evidence in the literature that $\mathrm{SiC}$ is also hemocompatible, N. Schettini of the USF SiC group conducted studies into the attachment and activation of blood platelets on various $\mathrm{SiC}$ surfaces [chapter in $\mathrm{SiC}$ book by Saddow]. What was significant about this work was that she prepared her platelet rich plasma (PRP) without the addition of any anticoagulating agents so as to clearly determine the interaction of PRP with $\mathrm{SiC}$ surfaces. In addition, she immersed the SiC samples, and Si control, for 15 min instead of 
the more common 5 min time period to clearly allow the clotting cascade to initiate. Fluorescent micrographs from her work [Figure 3] show that $3 \mathrm{C}-\mathrm{SiC}$ is more hemocompatible as indicated by less platelet adhesion and aggregation with circular shaped platelets, as compared to Si [32], reprinted with permission [Appendix A]. From both Dr. Coletti's and Dr. Schettini's results, it has been confirmed that Silicon Carbide ( $\mathrm{SiC})$ is both biocompatible and hemocompatible and, given that $\mathrm{SiC}$ is a semiconductor with known sensing potentiality, this material is a very promising candidate for in vivo biosensors.
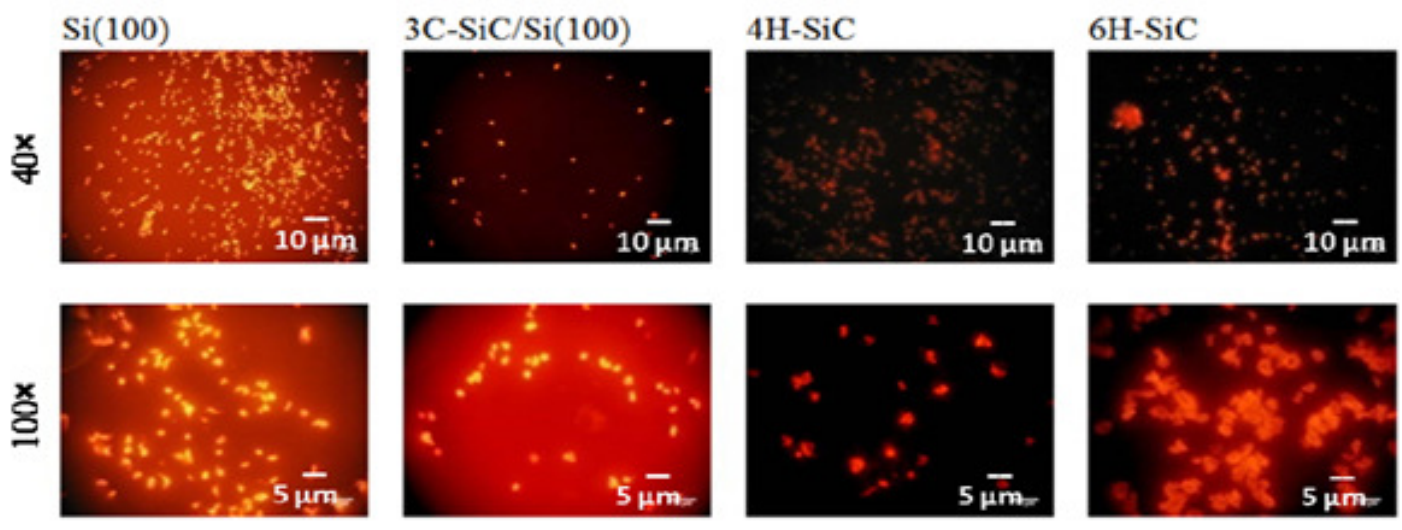

Figure 3: Flourescent micrographs of $\mathrm{Si}, 3 \mathrm{C}-, 4 \mathrm{H}-$, and $6 \mathrm{H}-\mathrm{SiC}$ showing platelet adhesion and aggregation. Note that $3 \mathrm{C}-\mathrm{SiC}$ shows less platelet adhesion and aggregation [32]

On the other hand, the B16 cells cultured on Si substrates appeared to be rounded and with a tendency to form clusters [Figure 2]. The apparent reduced contact area of the adherent cells confirms the lower biocompatibility of the Si substrates. HaCaT cells cultured on Si surfaces were morphologically similar to the control cells but generally a lower density of adherent cells was observed [Figure 2]. BJ cells typically present a lower density and reduced contact area when cultured on Si substrates. Fluorescent micrographs further show that $3 \mathrm{C}-\mathrm{SiC}$ is more hemocompatible as indicated by less platelet adhesion 
and aggregation with circular shaped platelets, as compared to Si in Figure 3 [32]. So it is found that Silicon Carbide $(\mathrm{SiC})$ material has both biocompatibility and great sensing potentiality which make it a very promising candidate for in vivo biosensors.

\subsection{Summary and Dissertation Organization}

In summary, it is important to develop a reliable, long-term in vivo continuous glucose monitoring sensor which would lessen the complications associated with diabetes through optimal glycemic control. For long term continuous glucose monitoring the impedance spectroscopy / electromagnetic coupling technique can be implemented using biocompatible implantable $\mathrm{SiC}$ based antenna sensing, where the resonant frequency shifts with different glucose levels.

This dissertation is organized into five chapters. Chapter 1 describes the objective and motivation of this research, background and review of current glucose sensors, a brief description of the glucose sensing mechanism using an electromagnetic radiation based antenna sensor, introduction of the material of choice, $\mathrm{SiC}$, for an in vivo biosensor (including this glucose sensor) and, finally the dissertation organization.

Chapter 2 presents an overview of the applicable antenna theory, the SiC based antenna sensor design for continuous glucose monitoring, and details of the different approaches towards realizing an all-SiC antenna. The approaches include heavily doped material growth using chemical vapor deposition and ion-implantation to achieve high doping concentration for the antenna radiation element. The fabrication procedure using standard photolithography is also been explained. Finally, different antenna responses are discussed and contrasted. 
Chapter 3 presents SiC-based metal patch (Ti/Au) antenna sensor performance. Using animal tissue the measured sensor response to fat and muscle in close proximity to the antenna is presented. Fatty tissue and blood mimicking liquids were prepared and the corresponding permittivity and conductivity measured to investigate the sensor's performance. In vitro sensor performance demonstrated that the sensor showed a dosedependent response to glucose concentration. Finally, in this chapter, the response of a $\mathrm{SiC}-\mathrm{Ti} / \mathrm{Au}$ antenna-based glucose sensor is discussed, showing almost linear dependence on glucose concentration in-vitro.

Chapter 4 presents an in-vivo experiment for foreign body response to subcutaneously-implanted devices in the pig. This in vivo study of different SiC-based antennas assesses the antenna sensor's foreign material cell/tissue interaction, biofouling, and studies surface modification to inhibit protein adsorption as well as other biocompatible material coatings. In this chapter, the details of the in vivo implantation procedure, histological tissue analysis, etc. is also discussed.

Chapter 5 presents the summary of the dissertation and some future direction of this work. The most important future work is scaling the antenna to operate at ISM/MISC bands. The radiation response of the all-SiC antenna can be improved by increasing the doping concentration. Finally, in this chapter, conclusions are drawn with a discussion of the major results of this investigation. 


\section{CHAPTER 2}

\section{ANTENNA DESIGN, MATERIAL GROWTH AND FABRICATION}

\subsection{Antenna}

Antennas are used for radiating and receiving electromagnetic energy. The official IEEE definition of an antenna states '...that part of a transmitting or receiving system that is designed to radiate or receive electromagnetic waves' [33]. For a simple conductor, time varying current, or an acceleration/deceleration of charge, creates radiation. So electromagnetic radiation is created due to a time varying electric and magnetic field which can be created with a sinusoidal voltage source. These electromagnetic waves are created continuously and travel through the transmission media, and radiated into free space. There are three different field regions surrounding the antenna and they are associated with two types of energies - radiating energy and reactive energy. The field regions are the reactive near-field region, the radiating near-field region (i.e., Fresnel Zone) and far field region (i.e., the Fraunhofer Zone). The antenna performance can be evaluated by a number of parameters. Some critical parameters are described here.

The radiation pattern, or radiation intensity, is defined as the power radiated from an antenna per unit solid angle [34]. Basically, the radiation pattern is a far field radiation property which is a plot of elevation angle $(\theta)$ and azimuthal angle $(\varphi)$. 
Directivity (D) is defined as "the ratio of the radiation intensity in a given direction from the antenna to the radiation intensity averaged over all directions" [34]. Generally, the directivity is expressed in $\mathrm{dBi}$ which indicates that the directivity is normalized to the radiation pattern of an isotropic radiator. It can also be estimated from the radiation pattern.

Input impedance - "the impedance presented by an antenna at its terminals or the ratio of the voltage to the current at the pair of terminals or the ratio of the appropriate components of the electric to magnetic fields at a point" [34]. The impedance of an antenna is expressed as:

$$
Z_{\text {in }}=R_{\text {in }}+j X_{\text {in }}
$$

where $Z_{\text {in }}$ is the antenna impedance; $R_{i n}$ is the antenna resistance and $X_{\text {in }}$ is the antenna reactance, respectively. The imaginary part of the input impedance $X_{\text {in }}$ is responsible for the power stored in the near field region. Resistive part $R_{i n}$ has two components - the first is the radiation resistance $\left(R_{r}\right)$, which is the power associated with this resistance that is actually the power radiated by the antenna. The second is the loss resistance $\left(R_{l}\right)$ which is associated with the power dissipation.

The Voltage Standing Wave Ratio (VSWR) is a measurement of the impedance mismatch of any RF structure. For maximum power transfer, the impedance $\left(Z_{\text {in }}\right)$ of the antenna has to be matched with the transmitter $\left(Z_{S}\right)$. If the impedance is not matched, some of the power is reflected back to the source which creates a standing wave that is characterized by VSWR. It is expressed as:

$$
V S W R=\frac{1+|\Gamma|}{1-|\Gamma|}
$$




$$
\Gamma=\frac{V_{r}}{V_{i}}=\frac{Z_{i n}}{Z_{S}}
$$

where $\Gamma$ is the voltage reflection coefficient, $V_{r}$ is the amplitude of the reflected wave and $V_{i}$ is the amplitude of the incident wave. In general most radio equipment is designed to have an input impedance of $50 \Omega$ so that impedance matching can be realized to this standard value.

Return loss (RL) is known as the amount of power that is lost (i.e., absorbed) in the load and not returned as a reflection. Similar to VSWR, the parameter RL also indicates the impedance matching of the transmitter and the antenna. When the transmitter and antenna impedance do not match, it creates standing waves.

$$
R L=-20 \log _{10}|\Gamma| \quad(\mathrm{dB})
$$

Perfect match means no power has been reflected, $\Gamma=0$ and as a result $R L=\infty$. On the other hand when all incident power is reflected, $\Gamma=1$ as a result $R L=0 \mathrm{~dB}$.

The antenna efficiency is a very important parameter that takes into account the amount of losses within the structure of the antenna and to the terminal of the antenna. These losses are due to conduction and dielectric $\left(I^{2} R\right)$ losses and the reflection due to the mismatch between transmitter and the antenna. Naturally the lower the conduction and dielectric losses better the antenna radiation efficiency. Antenna gain is related to the directivity of the antenna and always referenced to the main lobe of the radiation pattern. Gain is the amount of power that can be achieved in one major direction (main lobe) at the expense of other directions (minor lobes).

$$
G(\theta, \varphi)=e_{c d} D(\theta, \varphi) \quad(\mathrm{dBi})
$$

where $e_{c d}$ is called as the antenna radiation efficiency and $\mathrm{D}$ is the directivity. 
According to Balani [34], the bandwidth of an antenna is defined as "the range of usable frequencies within which the performance of the antenna, with respect to some characteristic, conforms to a specified standard." There are different types of antennas available and their shapes/sizes depend on their applications. The characteristics of an antenna are normally determined by its size, design (i.e., geometry) and the type of material used. We have selected patch antenna for this research with $\mathrm{SiC}$ as the insulating substrate.

\subsubsection{Theory of the Patch Antenna}

To evaluate the initial performance of a $\mathrm{SiC}$ antenna, simple microstrip patch antennas were selected because these antennas are low profile, conformable to planar and nonplanar surfaces, simple, inexpensive to manufacture, compatible with MMIC designs and, when the particular patch shape and mode are selected, they are very versatile in terms of resonant frequency, polarization, radiation pattern, and impedance. Although there are many variations on patch antenna design, the basic configuration, a rectangular patch, which is shown in Figure 4 (a), has been selected for this proposed research [34]. The microstrip patch antenna consists of a radiating patch on one side of a dielectric substrate and the other side of the substrate has a ground plane. The length, L, of a rectangular patch is usually $0.3333 \lambda_{0}<\mathrm{L}<0.5 \lambda_{0}$, where $\lambda_{0}$ is the free-space wavelength. Usually, the patch is selected to be very thin such as $t<<\lambda_{0}$ (where the patch thickness is t). Again the height $\mathrm{h}$ of the substrate is $0.003 \lambda_{0} \leq \mathrm{h} \leq 0.05 \lambda_{0}$. The typical range of relative dielectric constant of the substrate is $2.2 \leq \varepsilon_{\mathrm{r}} \leq 12$. In the patch antenna, two radiating slots are separated by the transmission line $\mathrm{L}$. The maximum radiation direction 
is normal to the plane of the patch antenna. The electric field distribution [Figure 4 (b)] shows that the electric field is zero at the center of the patch, maximum (positive) at one side, and minimum (negative) on the opposite side. The minimum and maximum continuously change sides according to the instantaneous phase of the applied signal. The electric field does not stop abruptly at the patch's periphery as in a cavity, rather, the fields extends into the outer periphery to some degree. These field extensions are known as fringing fields. . Primarily these fringing fields between the patch edge and the ground plane make the microstrip patch antenna radiate. A thick substrate with lower dielectric constant is desirable for good antenna performance since this provides better efficiency, radiation and larger bandwidth [34]. However, this type of configuration leads to a larger antenna size. Higher dielectric constants must be used to design a compact microstrip patch antenna; as a result the antenna will be less efficient, lower power and narrower bandwidth. Therefore a tradeoff exists between antenna dimensions and antenna performance.

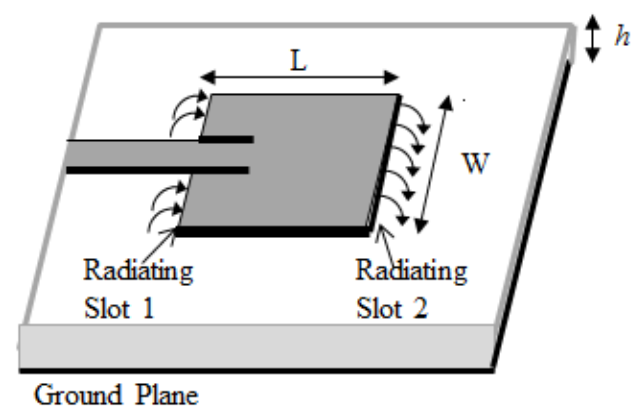

(a)

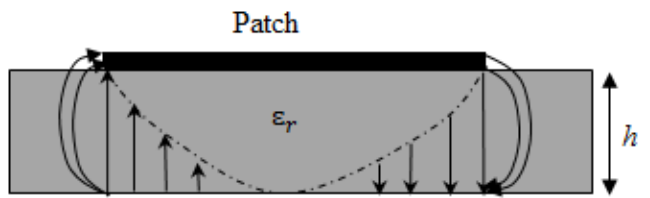

(b)

Figure 4: (a) Microstrip patch antenna, (b) electric field distribution (side view)

The antenna resonant length determines the resonant frequency and is about $\lambda_{0} / 2$ for a rectangular patch excited in its fundamental mode. The patch is, in fact, electrically a bit 
larger than its physical dimension due to the fringing fields. The amount of fringing is a function of the dimensions of the patch and the height of the substrate. For the principal E-plane (x-y plane) fringing is a function of the ratio of the length or width of the patch to the height of the substrate $(\mathrm{L} / \mathrm{h}$ or $\mathrm{W} / \mathrm{h})$ and the dielectric constant $\left(\varepsilon_{\mathrm{r}}\right)$ of the substrate. For patch antennas $L / h$ or $W / h>>1$, fringing is reduced, and for $\varepsilon_{\mathrm{r}}>>1$ the electric field lines are concentrated mostly in the substrate. The deviation between electrical and physical size is mainly dependent on substrate thickness and dielectric constant. For the dominant $\mathrm{TM}_{010}$ mode, the resonant frequency of a microstrip patch antenna is a function of its length, usually given by

$$
\left(f_{r}\right)=\frac{1}{2 L \sqrt{\epsilon_{r}} \sqrt{\mu_{0} \epsilon_{0}}}=\frac{\vartheta_{0}}{2 L \sqrt{\epsilon_{r}}}
$$

where, $\mathrm{L}$ is the length of the antenna, $\vartheta_{0}$ is the velocity of light and $\epsilon_{r}$ is the relative dielectric constant. Since above equation does not account for fringing, it must be modified to include edge effects and should be computed using

$$
\begin{gathered}
\left(f_{r}\right)_{101}=\frac{1}{2 L_{e f f} \sqrt{\epsilon_{e f f}} \sqrt{\mu_{0} \epsilon_{0}}}=\frac{1}{2(L+2 \Delta L) \sqrt{\mu_{0} \epsilon_{0}} \sqrt{\epsilon_{e f f}}} \\
=q \frac{1}{2 L \sqrt{\mu_{0} \epsilon_{0}} \sqrt{\epsilon_{r}}}=q \frac{\vartheta_{0}}{2 L \sqrt{\epsilon_{r}}} .
\end{gathered}
$$

The $q$ factor is referred to as the fringe factor (length reduction factor). As the substrate height increases, fringing also increases and leads to larger separations between the radiating edges and lower resonant frequencies.

The principal antenna parameter, the resonant frequency, depends on the relative dielectric constant, as well as conductivity of the material surrounding medium. Antenna performance can be described by four parameters; gain, return loss, efficiency, and operating bandwidth, which can be found by computing the electric field (E), magnetic 
field $(\mathrm{H})$, corresponding current/voltage distribution using Maxwell's equations, and applying boundary conditions. Gain is defined as the ratio of the radiated power in a particular direction to the total power accepted by the antenna. Return loss, defined as the ratio of reflected power to power accepted by the load, gives a useful measure of electrical power transfer efficiency. For maximum power transfer, the power source and load impedances must be matched [34]. There are many configurations that can be used to feed the patch antenna such as a microstrip transmission line, a coaxial cable, aperture coupling and proximity coupling are the most popular. With proximity coupling a conducting strip is connected directly to the edge of the microstrip patch. As compared to the patch, the strip is smaller in width with the advantage of this kind of feed arrangement being that it provides a planar structure. Without any matching element, the inset cut in the patch shown in Figure 4 is used to match the impedance of the feed line to the patch. The inset position is very crucial to achieve this matching. Sometimes, the radiation from the feed also leads to undesired cross polarized radiation [34]. However, this feed line not only provides the impedance matching but also eases the fabrication and simplicity in mathematical modeling. In our research the microstrip feed line was used for impedance matching because it is easy to fabricate and simple to match by controlling the inset position.

Designing 'embedded' antennas in lossy material like the human body is extremely challenging because of reduced antenna efficiency, impedance matching, the electrical impact of the surrounding environment, the need to reduce antenna size, and the very strong effect of multipath losses. In this research, the antenna was designed in air medium $\left(\varepsilon_{\mathrm{r}}=1\right)$ with the aim to implant this antenna in the fatty tissue region whose 
permittivity is low enough $\left(\varepsilon_{\mathrm{r}}=5\right)$ to operate in the body. Within the body, implants operate within certain frequency bands for medical applications but for this preliminary research and, due to the cost of semi-insulating $4 \mathrm{H}-\mathrm{SiC}$, the center frequency of the antenna was chosen to be $10 \mathrm{GHz}$ with the understanding that the antenna can be easily scaled to lower frequencies later by increasing the antenna dimensions properly.

\subsubsection{Frequency Band for Medical Applications}

Different communication commissions all over the world recommended different frequency bands for implantable medical devices. For example, the internationally recognized ISM (Industrial, Scientific and Medical) band, $2.45 \mathrm{GHz}$ is used for different medical applications. The U.S. Federal Communications Commission (FCC) has established the Medical Implant Communication Service (MICS) band, which is from 402 to $405 \mathrm{MHz}$, with a maximum band width of $3 \mathrm{KHz}$, which is reserved for ultralow power $(25 \mu \mathrm{W})$ communication with implanted medical devices [35]. The frequencies were chosen as a good compromise between antenna size and path loss within the body. Another subsection of the FCC bands allotted for this purpose is the Wireless Medical Telemetry Service (WMTS) band, which has frequency allotments of 608-614 MHz, 1395-1400 MHz, and 1427-1429 MHz. These frequencies cover a wide range of the electromagnetic spectrum and have very different in-vivo absorption characteristics. The FCC has an ongoing collaboration with the European Telecommunications Standard Institute (ETSI) to create legally allocated bands specifically for medical device communication. In this research, the design frequency was selected to be $10 \mathrm{GHz}$ to minimize the size and associated substrate costs. 


\subsection{SiC Based Antenna Simulations and Design}

A typical patch antenna structure consists of a single patch above a finite ground plane. There are various approaches to simulate patch antennas; in this study we have used the finite element method (FEM) via the Ansys High Frequency Structure Simulator (HFSS). HFSS - is a commercially developed package for electromagnetic modeling. The features of Ansys HFSS are the geometric model is divided into large number of tetrahedral and the collection of these tetrahedra is referred as the finite element mesh. FEM used by HFSS with an unknown vector quantity which is being used electromagnetic fields and currents. Sophisticated absorbing boundary condition at an artificial boundary is required by this FEM approach. For more accurate results, the structure is divided into larger number of tetrahedra and assuring internal convergence, as a result the execution time becomes very high. Ansys HFSS is typically used to model various homogeneous/inhomogeneous dielectric structures, which makes it appropriate simulation software for this research.

As mentioned earlier, in this research, initially the patch antenna was designed for 10 $\mathrm{GHz}$ just to make sure the antenna works properly on a SiC substrate. Also, the antenna length depends on resonance frequency shown in above equations; higher frequency corresponds to smaller length. Depending on the $10 \mathrm{GHz} \mathrm{SiC}$ antenna performance, future work will include optimization of the antenna structure to get desirable response and resonance frequency. The Ansys HFSS software has been used for SiC antenna design and simulation of the antenna performance such as return loss and radiation pattern. The length and width of the antenna were calculated based on the resonant frequency and effective dielectric constant of the substrate. The feed length was selected 
carefully for impedance matching purposes. The geometrical design parameters were determined based on numerical simulations using Ansys (HFSS). The proposed sensor is rectangular in shape with dimensions of $8.5 \mathrm{~mm}$ width, $11.5 \mathrm{~mm}$ length, and $0.37 \mathrm{~mm}$ height. The detailed structure of this $10 \mathrm{GHz}$ antenna sensor is shown in Figure 5 (a). A $4 \mathrm{H}-\mathrm{SiC}$ semi-insulating substrate is an ideal substrate for the antenna because it has no microwave (i.e., RF) signal loss in thus yielding the maximum possible antenna gain. The simulated radiation pattern is shown in Figure 5 (b).

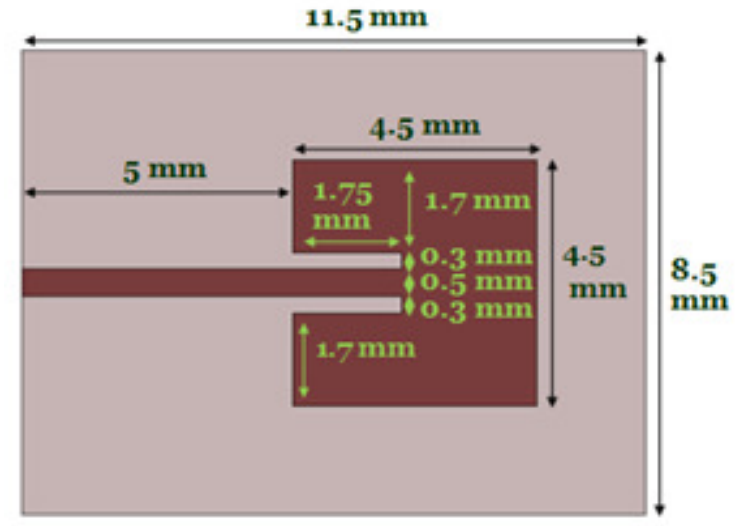

(a)

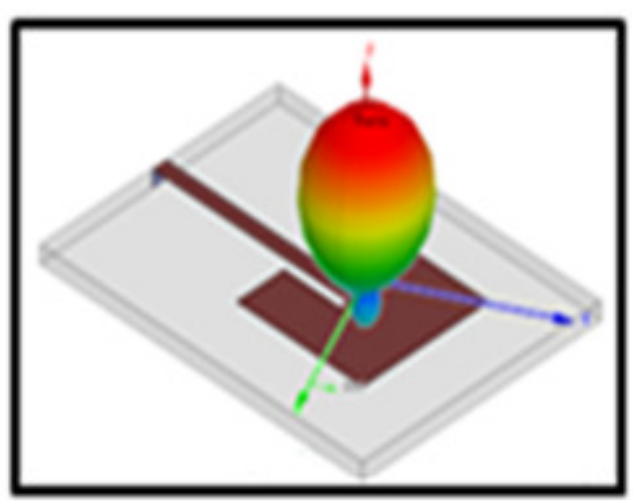

(b)

Figure 5: (a) Schematic of the $\mathrm{SiC}$ based antenna sensor; (b) simulated radiation pattern

One of the most important parameters of an antenna is its return loss. Figure 6 (a) shows the simulated return loss of the all-SiC antenna for a $10 \mathrm{GHz}$ antenna structure. Another important antenna parameter is the radiation efficiency. The efficiency increases with semiconductor conductivity which is directly proportional to the doping level of the material. The radiation efficiency also increases with substrate thickness, which is shown in Figure 6 (b). 


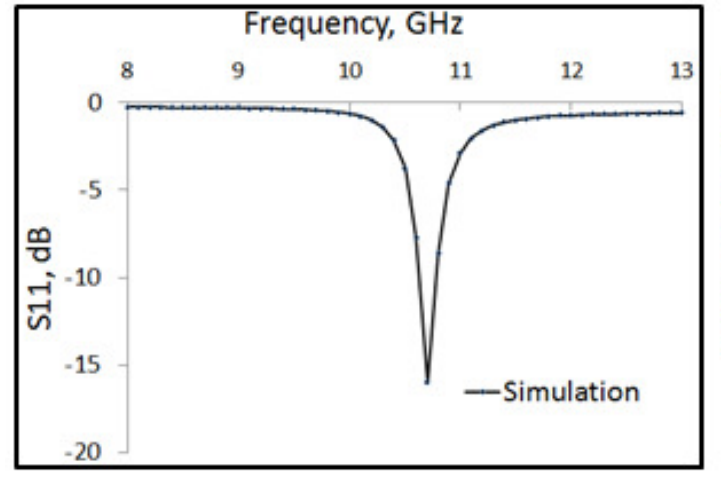

(a)

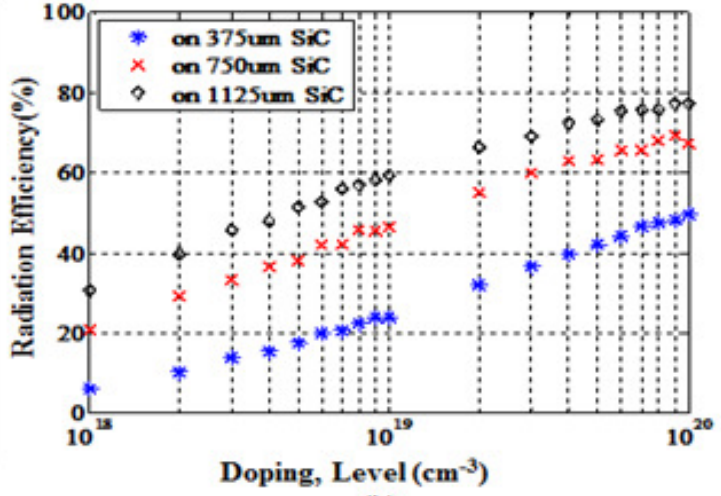

(b)

Figure 6: (a) All SiC antenna return loss; (b) dependence of radiation efficiency on doping level and substrate thickness

The metallic-like behavior of the $\mathrm{SiC}$ material will also allow it to act as the antenna radiating patch element. The conductivity of $\mathrm{SiC}$ primarily depends on the doping concentration of the material, from the following equation

$$
\sigma=q \mu N
$$

where $\sigma$ is the conductivity in $\mathrm{S} / \mathrm{m}, \mathrm{q}$ is the electric charge in Coulombs, $\mu$ is the mobility in $\mathrm{cm}^{2} / \mathrm{V}$-s and $\mathrm{N}$ is the doping concentration in $\mathrm{cm}^{-3}$.

In a semiconductor like $\mathrm{SiC}$, this doping has been achieved by replacing some lattice atoms such as Carbon atoms by suitable doping atoms like Nitrogen atom. Due to the appropriate donor levels in the bandgap of $\mathrm{SiC}$ and small difference in lattice constants with the Carbon atoms, $\mathrm{N}_{2}$ is commonly used as the $\mathrm{n}$ type dopant in $\mathrm{SiC}$. A pure neutral semiconductor is known as intrinsic because the electron (n) and hole (p) concentration is equal and the corresponding intrinsic Fermi level is in the middle of the energy band gap [36],

$$
n=p=n_{i}=\left(N_{c} N_{v}\right)^{1 / 2} \exp \left(-\frac{E_{g}}{2 k_{B} T}\right)
$$


where, $N_{c}$ and $N_{v}$ is effective density of states for conduction and valance band respectively. Here, $E_{g}$ is the band gap energy in electron-volt $(\mathrm{eV})$ and thermal energy, $k_{B} T$ also expressed in $\mathrm{eV}$. This Fermi level is changed by introducing impurities which supply either electron known as donors or n-type dopants (donates an electron) or hole known as acceptor (accepts an electron) or p-type dopants. The antenna electrode of this research was doped with n-type dopants. Therefore, we will only refer to the donor level (n-type). The shallow donors are fully ionized as ionization energies are smaller than or close to the thermal energy [36]. At charge neutrality -

$$
p+N_{d}^{+}-N_{a}^{-}-n=0
$$

where, $N_{d}^{+}$and $N_{a}^{-}$are the ionized donors and acceptors concentrations respectively. If the semiconductor $(\mathrm{SiC})$ is doped with donors $\left(\mathrm{N}_{2}\right), N_{a}^{-}=0$. Therefore,

$$
n=p+N_{d}^{+}
$$

When the ionized donor concentration is a few orders of magnitude higher, the hole concentration can be neglected. Another important parameter is the mobility ( $\mu$, unit: $\mathrm{m}^{2} /$ V.s). Scattering at crystal defects like dislocation or impurity (unwanted) atoms leads to decreasing mobility with increasing doping concentration. Some additional parameters such as temperature, mass, life time (holes/electron) are responsible for the differences in mobility, conductivity of a semiconductor. Using impurity incorporation into $\mathrm{SiC}$ material, an antenna electrode will be realized using semiconductor material.

\subsubsection{SiC Based Ti/Au Metal Antenna}

The simulation of the same antenna structure shows that a biocompatible all - SiC design would work as well as a metal (Ti/Au) approach. To verify the simulation, the 
antenna performance on a SiC substrate (Ti/Au as metal patch) was fabricated. The metal patch antenna was fabricated using a semi-insulating $4 \mathrm{H}-\mathrm{SiC}$ substrate. A high dielectric constant $\left(\varepsilon_{\mathrm{r}} \sim 10\right)$ semi-insulating $4 \mathrm{H}-\mathrm{SiC}$ semiconductor substrate was purchased for this work. Standard photolithography and e-beam evaporation were used for fabrication and metal deposition, which is shown in Figure 7 (a). The fabricated antenna is shown in Figure 7 (b).

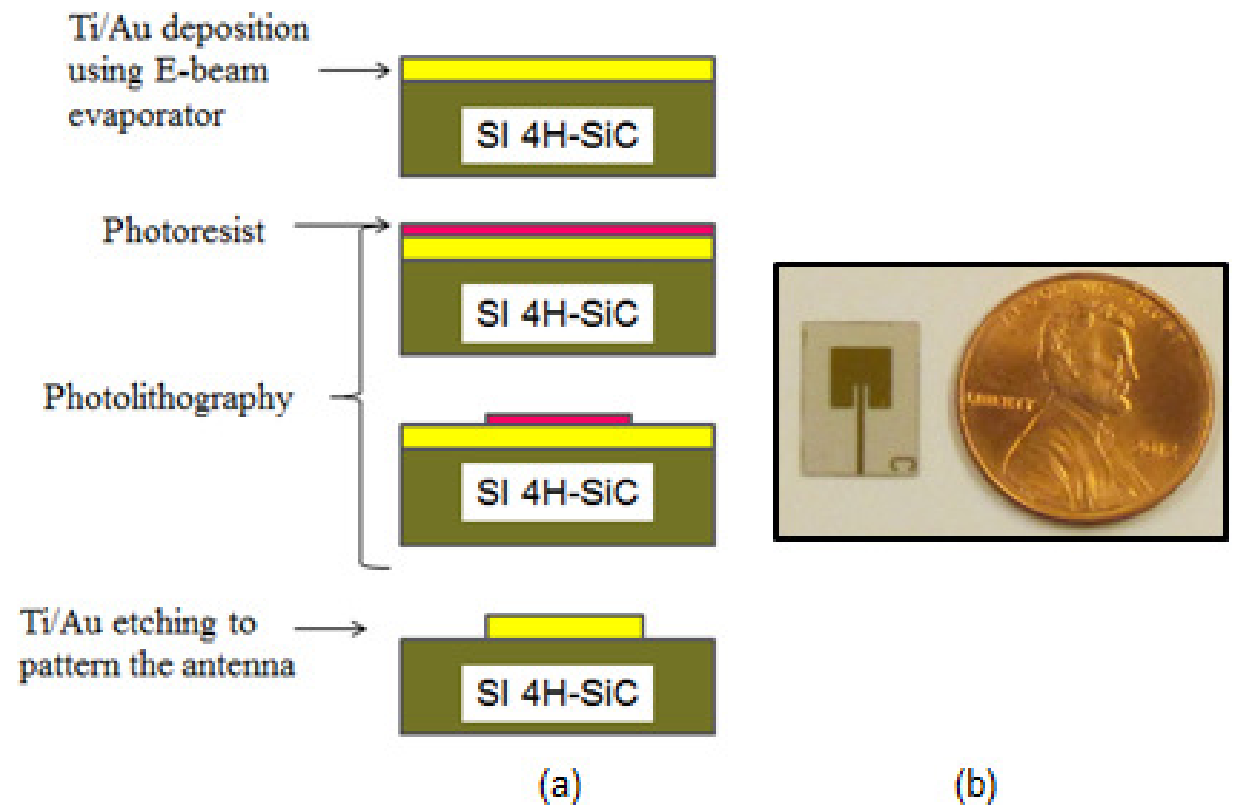

Figure 7: (a) Fabrication process; and (b) photograph of the Ti/Au patch on semiinsulating $4 \mathrm{H}-\mathrm{SiC}$ antenna

A simulation of the patch structure using a highly conductive metal (Ti/Au) patch supports the hypothesis that a biocompatible, heavily-doped $\left(10^{20} \mathrm{~cm}^{-3}\right) \mathrm{SiC}$ patch design would work as an antenna. In order to function, the sensor antenna requires an input RF signal which is provided by the Vector Network Analyzer (VNA).

The HP 8719D VNA at the USF WAMI Center was used to measure the Ti/Au antenna performance - resonance frequency, bandwidth, $S_{11}$ response - which is shown in 
Figure 8 along with the simulated data. The response of the fabricated Ti/Au antenna is better in terms of targeted resonance frequency of $10 \mathrm{GHz}$ and return loss $\mathrm{S}_{11}$ as compared to the simulated data. This Ti/Au patch antenna was fabricated using a semiinsulating 4H-SiC substrate to serve as a "baseline" antenna, which also provides a point of reference for the non-metallic, all-SiC antenna.

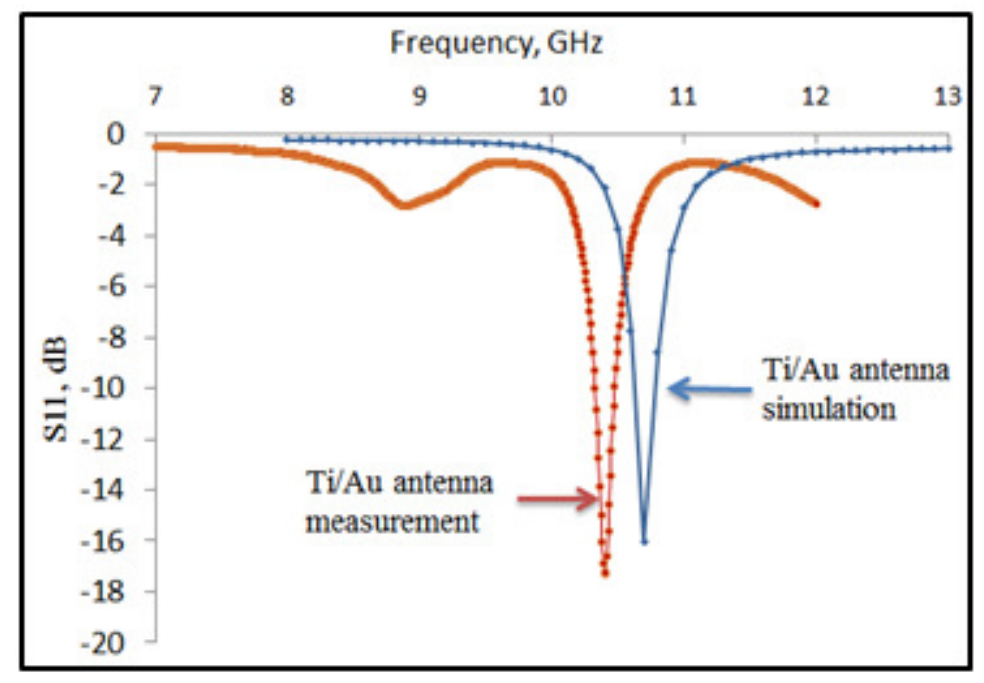

Figure 8: Simulation and experimental return loss of Ti/Au antenna

\subsubsection{Different SiC Based Antenna Designs and Methodologies}

Before explaining different methodologies to realize an all-SiC antenna structure, we need to know our minimum goal to achieve a reasonable antenna response. Here, the antenna electrode - heavily doped SiC top layer - is the most important part for the antenna to radiate. The simulation result of different dopant levels shown in Figure 9 indicates that a doping concentration of $5 \times 10^{19} \mathrm{~cm}^{-3}$ can give a reasonable antenna response. This response can be improved by increasing substrate height (i.e., thickness). As stated before, the diffusion process of impurity incorporation into SiC is extremely shallow, but options for dopant incorporation include in-situ doping during growth of the 
material or ion-implantation and subsequent annealing after growth. For in-situ n-type doping, typically Nitrogen $\left(\mathrm{N}_{2}\right)$ and Ammonia $\left(\mathrm{NH}_{3}\right)$ gases are used. It is also possible to obtain heavily doped $\mathrm{SiC}$ by ion implantation [37].

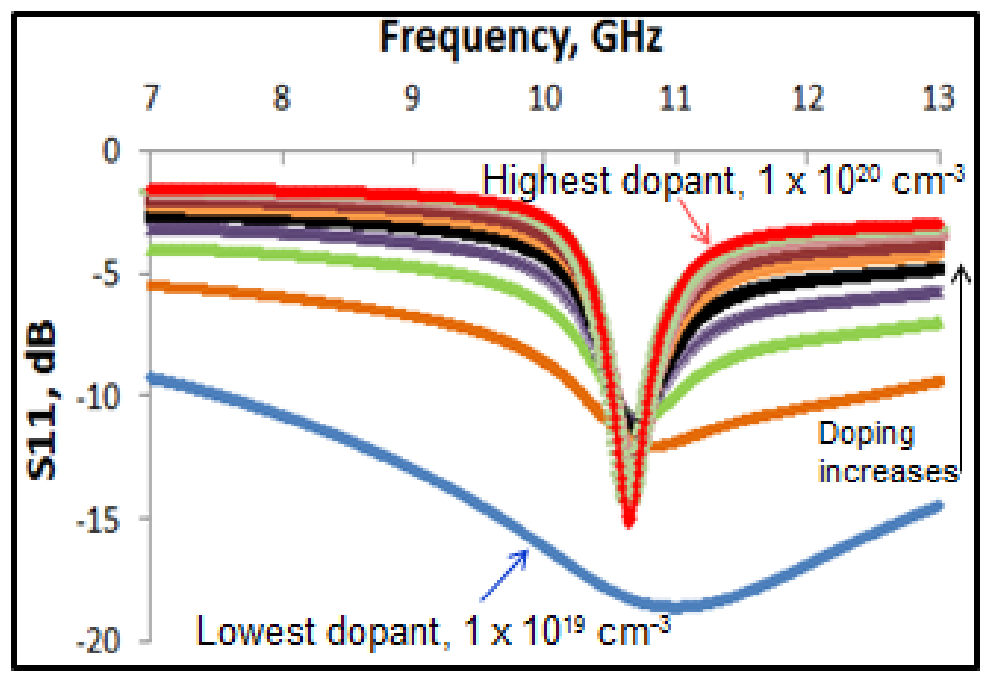

Figure 9: Simulation of antenna response for $1 \times 10^{19}$ to $1 \times 10^{20} \mathrm{~cm}^{-3}$ doping concentration. Return loss of $\mathrm{SiC}$ based $\mathrm{Ti} / \mathrm{Au}$ antenna is plotted vs. frequency. Simulations performed using HFSS

These doping methodologies will be used in the investigation of three $\mathrm{SiC}$ based structures as presented in Figure 10.

Approach (a) investigates a 4H-SiC/4H-SiC structure. 4H-SiC semi-insulating wafers were used as substrates to grow highly-doped $4 \mathrm{H}-\mathrm{SiC}$ epitaxial layers using the MF1 hotwall Chemical Vapor Deposition (CVD) system in the USF SiC Group. The process of doping the $\mathrm{SiC}$ layers requires additional gas precursors, for $\mathrm{n}$-type doing - nitrogen $\left(\mathrm{N}_{2}\right)$. One of the benefits of $\mathrm{SiC}$ growth via hot-wall $\mathrm{CVD}$ is the temperature processing flexibility. Not only can the CVD reactor go to high processing temperatures (in excess of $2000^{\circ} \mathrm{C}$ ), but an identical design, with different mechanical components, can be used for lower-temperature processing, where flow rates and other parameters are adjusted. 
After growing the highly doped epilayer at elevated temperatures, the device will be fabricated using a designed antenna mask, where the patterned doped epilayer acts as an antenna electrode. This is accomplished by dry reactive ion etching (DRIE) of the nonmetallic patch area thus forming the desired patch geometry shown in Figure 5. Approach (b) investigates a $3 \mathrm{C}-\mathrm{SiC} / \mathrm{Poly} \mathrm{Si} / \mathrm{SiO} 2 / 4 \mathrm{H}-\mathrm{SiC}$ structure. This is also an etch-back approach involving highly doped polycrystalline $3 \mathrm{C}-\mathrm{SiC}$ (poly-SiC) epitaxial growth and patterning. The concept realizes a radio frequency (RF) sensor using a $\mathrm{SiC}$ antenna, which includes a semi-insulating 4H-SiC substrate, $\mathrm{SiO} 2$, Poly $\mathrm{Si}$ and a doped polycrystalline 3C-SiC layer. We collaborated with the USF NREC to grow the $\mathrm{SiO} 2$ layers and CVD growth of poly Si. Poly 3C-SiC was then grown on these surfaces using the same CVD reactor as sensor (a) described above.

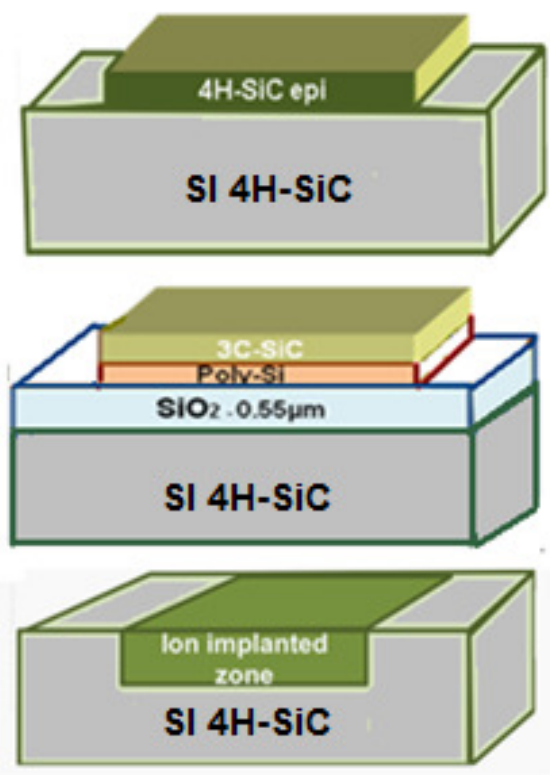

Figure 10: Cross section of three envisioned approaches to realize fully biocompatible glucose sensor using $\mathrm{SiC}$ antenna. (a) Approach a: etch - back approach involving highly doped epitaxial growth and patterning; (b) Approach b: poly-3C-SiC on insulator; (c) Approach c: fully planar approach using ion- implantation and thermal activation 
Approach (c) investigates an implanted $4 \mathrm{H}-\mathrm{SiC} / 4 \mathrm{H}-\mathrm{SiC}$ structure. It is a fully planar approach where semi-insulating $4 \mathrm{H}-\mathrm{SiC}$ would is used as the substrate. To achieve a heavily doped antenna region (metal like conductivity), ion implantation is applied to the 4H-SiC material. After patterning the antenna implant mask, ion-implantation is performed at a high enough energy to synthesize a degenerate SiC layer to form the antenna electrode structure. The same (CVD) reactor is then used to anneal the ionimplanted $\mathrm{SiC}$ layers in order to activate the dopants and reduce the crystal damage caused by the implantation.

High quality, heavily doped $\mathrm{SiC}$ material is crucial for all of the $\mathrm{SiC}$ antennas discussed here. Uniform, high quality epitaxial layers are produced when needed to realize the heavily doped layer, either of single or polycrystalline structure. This CVD

process is ideal for making antenna structures on semi-insulating substrates for the creation of an all-SiC, completely bio-compatible, sensor for glucose monitoring and other bio-medical applications. The schematic diagram of all approaches is shown in Figure 10.

\subsection{Chemical Vapor Deposition (CVD) Growth}

A solid film is formed onto a surface or substrate by a chemical reaction emanating from vapor phase precursors using a technique known as chemical vapor deposition (CVD). Generally the chemical reactions activated by ohmic heating, RF induction heating, light, or plasma. Often this technique employed for the growth of high quality and uniform thin films. To develop a solid film, this CVD technique involves a series of sequential steps beginning with the vapor phase and progressing through a series of quasi 
steady-state reactions [38]. This progression of thin film growth can be summarized by the following sequence of events. First, the reactant gases diffuse through the boundary layer to the surface. Second, the precursors transport toward the deposition surface or reaction site (i.e., dangling chemical bond) and the reactants adsorb on the surface. Third, a chemical reaction happens to the reactants which may be catalyzed by the surface. Fourth, the reaction by-products undergo desorption from the surface. Fifth, these byproducts diffuse through the boundary layer, enter the main gas stream for exhaust out of the reactor. Finally, the condensed product on the substrate is incorporated into the structure of the developing film. Although there are many rate-limiting steps are known to exist, mass transport and surface kinetics primarily administered the deposition rate of CVD processes. Several process parameters control these two rate limiting steps. The pressure and temperature of the growth environment greatly impact the deposition process. The thickness of the boundary layer mainly controlled by the pressure, and, as a result, affects the rate of the reactant and product diffusion. The boundary layer is thinner at low pressure which minimizes the diffusion time across the region. This is known as a transport-limited CVD regime; where the deposition rate is limited by the diffusion of reactants to the surface and is less sensitive to temperature. At low temperature, an oversupply of reactants is created due to the molecules reacting slowly. In contrast, if the temperature is high, the surface reactions take place quickly and the reaction rate is limited by the diffusion of molecules. The growth regime (transport-limited or surface reaction-limited) is determined by the slowest process (diffusion or chemical reaction) [39]. 
Gas velocity is the other important process parameter that influences reaction rate. The CVD process involves the transport of precursor gases through the use of a carrier gas, which usually a laminar flow although occasionally has some turbulence. When a fluid flows over a stationary surface, a thin layer of fluid immediately above the surface is stationary which is known as the boundary layer and is inversely proportional to the gas velocity and directly proportional to the fluid viscosity and pressure. The boundary layer increases along the direction of the carrier gas flow in a horizontal CVD reaction, which leads to an exponential decrease in the deposition. The gas velocity increases by tilting the susceptor by continuously decreasing cross-sectional area and thus reduces the thickness of the boundary layer along the flow direction.

Independent of the type of film growth technique, the initial stages of film growth is characterized by three major phenomena. First, the material must condense out of the vapor phase and nucleate on a substrate. Second, the reactant species impinging on the surface is attracted to the substrate by the London dispersion forces of the substrate atoms. The probability that an impinging atom will be adsorbed onto the surface is related to the sticking coefficient, which is the ratio of the amount of material condensed on the surface to the total amount of impinging atoms [38]. When an atom is adsorbed onto the surface, it must overcome the surface binding energy, Qdesorb, in order to leave the surface. Given the length of time, $\tau_{\mathrm{s}}$, which an atom stays on the surface, the vibrational frequency, $v$, of the adsorbed atom, is expressed by,

$$
\tau_{s}=\frac{1}{\vartheta} \exp \left(\frac{Q_{\text {desorb }}}{k T}\right)
$$

when $k T$ is small in comparison to $Q_{\text {desorb }}$, the adsorbed atom will spend longer time on the surface, so the chance of the atom being incorporated on the surface is high. When the 
surface atoms energy is on the order of $k T$, then the adsorbed atom will have a high probability of being desorbed. The condensed atoms or molecules tend to aggregate and form small clusters on the surface of the substrate when incorporated on to the surface, this process is called nucleation. These small clusters are in a constant free energy struggle between the releasing free energy when forming a cluster and having to pay energy cost when forming a surface interface between two distinct phases. The small clusters are unstable when the energy released from the formation of its volume cannot sustain the creation of its surface. As soon as the clusters have reached a critical size, any additional molecules to the cluster releases energy instead of costing energy and nucleation growth can be sustained. Therefore, randomly formed nucleation sites reach a saturation density and undergo island coalescence via diffusion and continuing capture of adatoms.

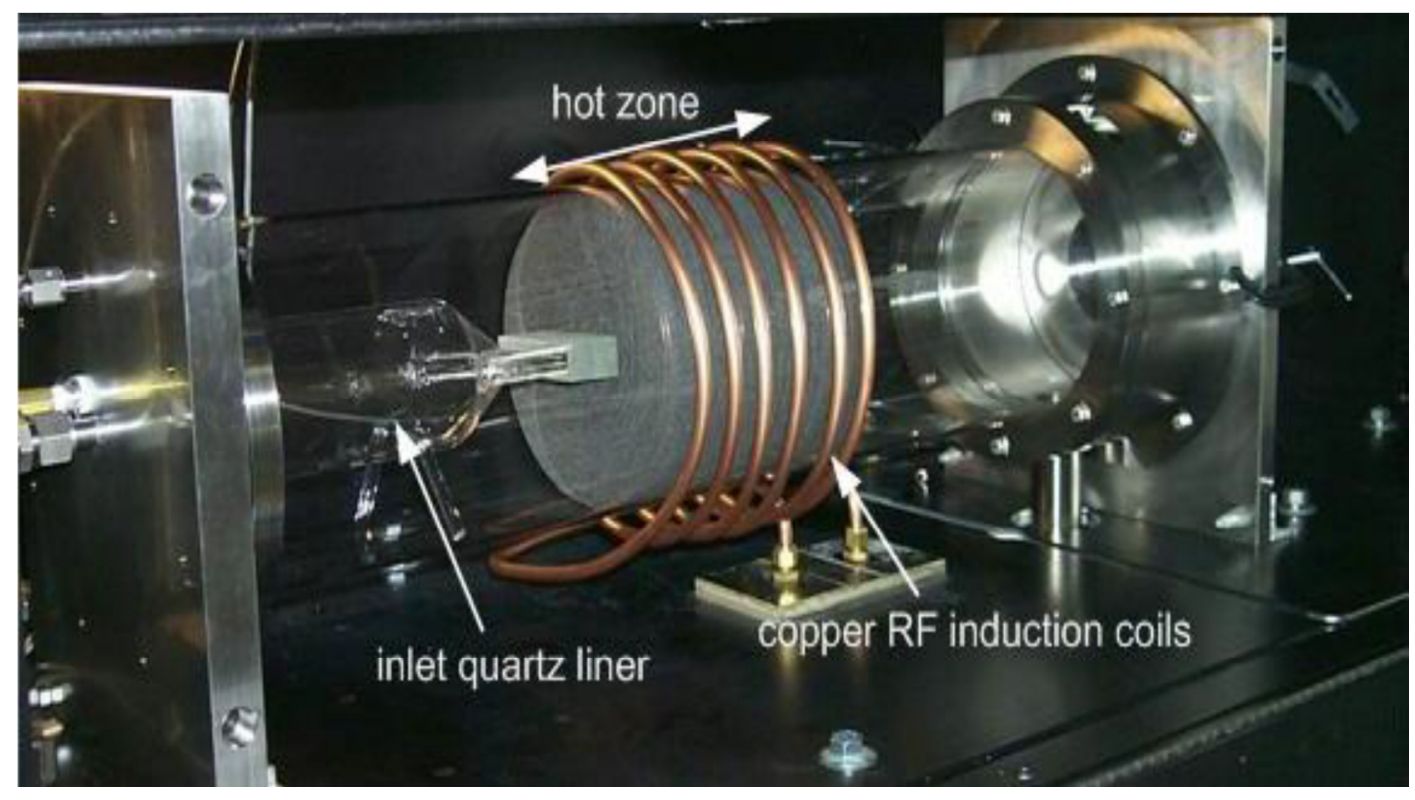

Figure 11: Photograph of the MF2 CVD horizontal reactor at USF. MF2 was used for the growth of all films reported in this thesis and is dedicated solely for $3 \mathrm{C}-\mathrm{SiC}$ on $\mathrm{Si}$ growth and processing 
When the internuclear distances are on the order of the mean surface diffusion length, saturation occurs. As the islands grow, a connected network form by assimilate subcritical nuclei and coalesce with other islands. Eventually, a steady-state growth above the first layer occurs. However, in CVD growth process, an additional step - a chemical reaction among the surface-adsorbed reactants occurs at the gas-substrate interface. A majority of CVD reactions are endothermic which means they must usually wait until they interact with the heated substrate while a simple condensation is always exothermic. The other important feature of the CVD process that complicates this growth sequence is that the intrinsic impurities, as reaction products, need to be considered in the vicinity of the film growth (Sivaram, S 1995). The CVD reactor, known as MF2 at USF is shown in Figure 11.

\subsubsection{Epi-growth of $4 \mathrm{H}-\mathrm{SiC}$ on $\mathrm{SI} 4 \mathrm{H}-\mathrm{SiC}$, Approach (a)}

Initial research on approach (a), namely the epi etch back approach involving highly doped homo-epitaxial growth and pattering was started by purchasing a comparatively low cost n-type $4 \mathrm{H}-\mathrm{SiC}$ semiconductor substrate to optimize the growth parameters. Following a standard cleaning the $4 \mathrm{H}-\mathrm{SiC}$ substrate was placed in a horizontal, hot-wall reactor heated by the $\mathrm{RF}$ induction of a $\mathrm{SiC}$-coated graphite susceptor. The wafer was loaded into a molded poly-SiC plate to fix the position of the wafer within the reactor hot zone. This polyplate was then seated into a recess in the susceptor and the chamber was sealed and evacuated of residual gases. The chamber was then filled with palladiumpurified hydrogen. The corresponding growth pressure and temperature was 150 Torr and $1560^{\circ} \mathrm{C}$ where the silicon to carbon ratio, $\mathrm{Si} / \mathrm{C}$, for the growth stage was 1.3 . The 
standard gases used for $4 \mathrm{H}-\mathrm{SiC}$ growth are: palladium-purified hydrogen, $\mathrm{H}_{2}$, which is used as the transport gas; propane $\left(\mathrm{C}_{3} \mathrm{H}_{8}\right)$, which is the carbon precursor; and a $10 \%$ silane $\left(\mathrm{SiH}_{4}\right)$ premixed in $90 \%$ hydrogen ballast $\left(\mathrm{H}_{2}\right)$, which is the silicon precursor. All the gases flow was maintained with mass flow controllers (MFCs). Details of the growth process may be found in the dissertation of Dr. R. Myers-Ward, formerly of the USF SiC Group [40]. More than thirty epitaxial films were grown using the MF1 hot wall reactor to achieve a doping concentration as high as possible for the antenna structure. Nitrogen gas was used as the n-type dopant. To obtain a better doping density it has been elegantly demonstrated that by changing the $\mathrm{Si} / \mathrm{C}$ ratio of the input gases the doping concentration will change without changing the flow of Nitrogen gas. This effect is known as site competition [37]. Unfortunately, when changing the stoichiometry, the morphology is affected somewhat and it is therefore not always desirable to do this. In this research the films were grown using this site competition where $\mathrm{Si} / \mathrm{C}$ ratio has been changed.

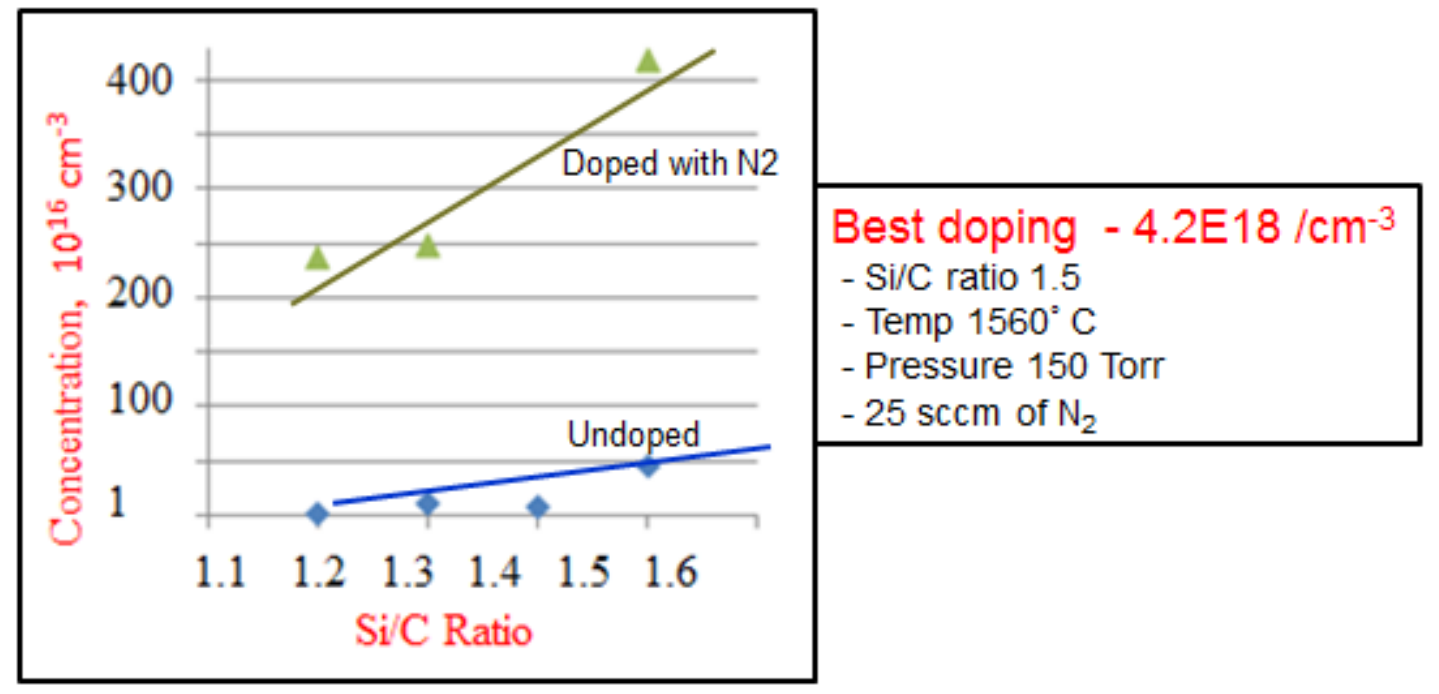

Figure 12: Measured doping concentration increased with Silicon/Carbon (Si/C) ratio; the growth condition of the best film is also shown 
The best film in terms of quality and doping concentration $\left(4.2 \times 10^{18} \mathrm{~cm}^{-3}\right)$ was achieved at a pressure, temperature and $\mathrm{Si} / \mathrm{C}$ ratio of 150 Torr, $1560^{\circ} \mathrm{C}$ and 1.5 respectively where the Nitrogen flow was kept constant at $25 \mathrm{sccm}$ which is shown in Figure 12. T The doping density is shown in Figure 12. The doping concentration was measured with a $\mathrm{Hg}$ probe station using a Capacitance-Voltage $(\mathrm{CV})$ measurement system (Kithley $590 \mathrm{CV}$ analyzer). The film surface morphology was very smooth and mirror- like.

After optimization of the growth parameters for the doped $4 \mathrm{H}-\mathrm{SiC}$ epitaxial thin films, a semi-insulating (SI) 4H-SiC wafer was purchased for the antenna substrate which has high dielectric constant and resistivity. The same epitaxial film growth process as outlined above was used to grow a heavily-doped film on the semi-insulating substrate using the optimized parameters. The morphology was not as good as the film on semiconductor. Different parameters have been used on semi-insulating substrate but unfortunately, when changing the stoichiometry, the morphology was affected somewhat. The idea was to grow highly n-doped epitaxial layer of $4 \mathrm{H}-\mathrm{SiC}$ on semi-insulating substrate and antenna structure obtained by etching the conductive film back to the substrate using standard photolithography and DRIE etching. Upon further evaluation of the first approach, structure (a), it was concluded that the morphological degradation of the $4 \mathrm{H}-\mathrm{SiC}$ material on the on-axis $\mathrm{SI} 4 \mathrm{H}-\mathrm{SiC}$ substrate would adversely affect the device results. It is proposed in future work that a costly off-axis SI 4H-SiC substrate could offer improvements for this structure option. In fact there is no reason, based on the literature where doping levels of $10^{20} \mathrm{~cm}^{-3}$ are routinely achieved during the growth of $4 \mathrm{H}-\mathrm{SiC}$ 
[41]. And due to the high quality of the doped layer this approach to realizing the antenna should yield the best performance.

\subsubsection{Growth of Poly 3C-SiC on SI 4H-SiC, Approach (b)}

This is a low temperature growth process compared with single-crystalline $3 \mathrm{C}-\mathrm{SiC}$ on $\mathrm{Si}$ epi growth, where n-doped poly $3 \mathrm{C}-\mathrm{SiC}$ was served as an antenna electrode. After cleaning the SI 4H-SiC substrate, a $\mathrm{SiO}_{2}(0.5-5 \mu \mathrm{m})$ film was deposited using PECVD (Plasma Enhanced Chemical Vapor Deposition) followed by a $100 \mathrm{~nm}$ thick layer of poly Si which was deposited by standard LPCVD processing. This poly-Si film was used as a seed layer for the poly 3C-SiC layer [42]. The 3C-SiC process developed for this reactor involves two main process stages, namely the carbonization and growth stages [Reyes, Shishkin, et al. 2005, 43]. The pressure for the carbonization process was 400 Torr, and growth pressure was 550 Torr. The same standard gases used for this poly 3C-SiC growth palladium-purified hydrogen $\mathrm{H}_{2}$, which is used as the transport gas; propane $\left(\mathrm{C}_{3} \mathrm{H}_{8}\right)$, which is the carbon precursor; and a $10 \%$ silane $\left(\mathrm{SiH}_{4}\right)$ premixed in $90 \%$ hydrogen $\left(\mathrm{H}_{2}\right)$, which is the silicon precursor. The carbonization stage occurred while the sample temperature was ramped to $1135^{\circ} \mathrm{C}$ at a rate of $\sim 35^{\circ} \mathrm{C} / \mathrm{min}$.

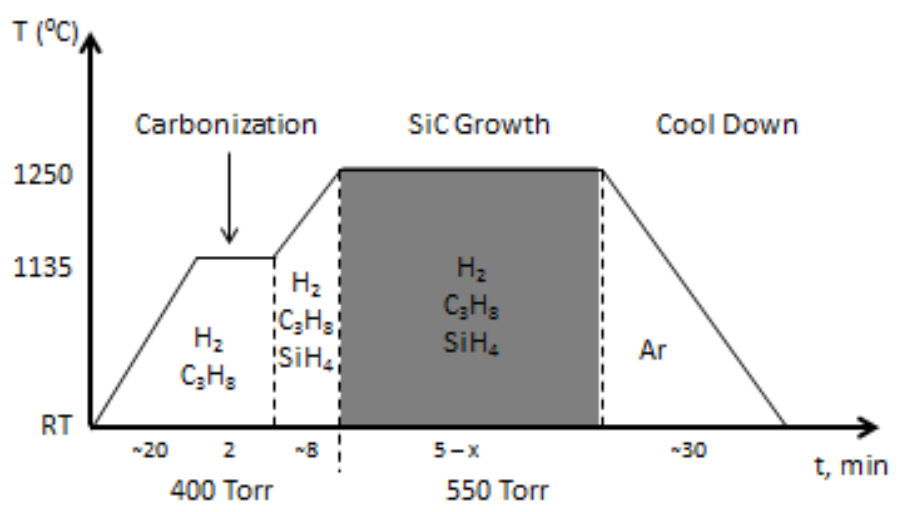

Figure 13: CVD growth process schedule used for approach (b) 
Throughout the temperature ramp a flow of $16 \mathrm{sccm}$ of $\mathrm{C}_{3} \mathrm{H}_{8}$ was maintained with a mass flow controller (MFC), and the $\mathrm{H}_{2}$ carrier gas flow was maintained at $20 \mathrm{slm}$. Once the carbonization temperature was reached $\left(1135^{\circ} \mathrm{C}\right)$, the temperature was maintained for 2 min to allow conversion of the poly $\mathrm{Si}$ surface into poly $3 \mathrm{C}-\mathrm{SiC}$. After carbonization and creation of the poly $3 \mathrm{C}-\mathrm{SiC}$ template layer, the temperature was ramped a second time at a rate of $35^{\circ} \mathrm{C} / \mathrm{min}$ to the growth temperature of $1250^{\circ} \mathrm{C}$. During this ramp, we determined that it is advantageous to decrease the flow of $\mathrm{C}_{3} \mathrm{H}_{8}$ while simultaneously introducing and increasing the flow of $10 \% \mathrm{SiH}_{4} / 90 \% \mathrm{H}_{2}$ in a step-wise manner. At the growth temperature the input gas silicon to carbon ratio, $\mathrm{Si} / \mathrm{C}$, for the growth stage was 0.35. $\mathrm{H}_{2}$ flow was maintained at $20 \mathrm{slm}$ until $30^{\circ} \mathrm{C}$ before the ramp was completed, where it was increased to $30 \mathrm{slm}$, and the pressure was increased from 400 Torr to 550 Torr. The temperature and gas flows were then held constant, allowing the continued epitaxial growth of poly $3 \mathrm{C}-\mathrm{SiC}$ on the carbonized poly-Si. Figure 13 graphically summarizes the process.

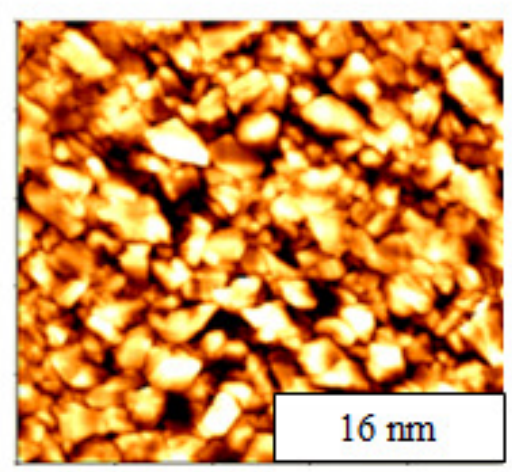

(a)

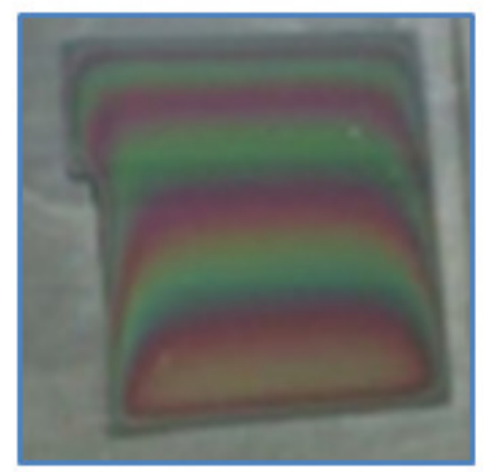

(b)

Figure 14: AFM morphology of doped poly 3C-SiC (a) 5 x $5 \mu \mathrm{m}^{2}$ with a surface roughness of $16 \mathrm{~nm}$ RMS. (b) epilayer photograph: 1 hour 40 minute growth. Poly-3CSiC film thickness $1.5 \mathrm{um}$. Die size 15 by $12 \mathrm{~mm}$ [44] 
The initial test dies yielded a hazy surface over the standard test samples. A series of experiments were conducted in order to obtain a uniform specular film deposition within the growth zone. In order to develop an optimized process only one growth parameter was changed at a time while all others are held constant. The time of film growth was set at 30 minutes for all tests since thin polycrystalline films are difficult to discern from thin mono-crystalline films in the early stages of growth. A series of experiments were performed where the $\mathrm{Si} / \mathrm{C}$ ratio was varied in increments of 0.2 from an initial value of 1.4 to 0.2 .

The best resulting film morphology was clear with large interference fringes (as seen in Figure 14). Nitrogen gas was also used as n-type dopant source.

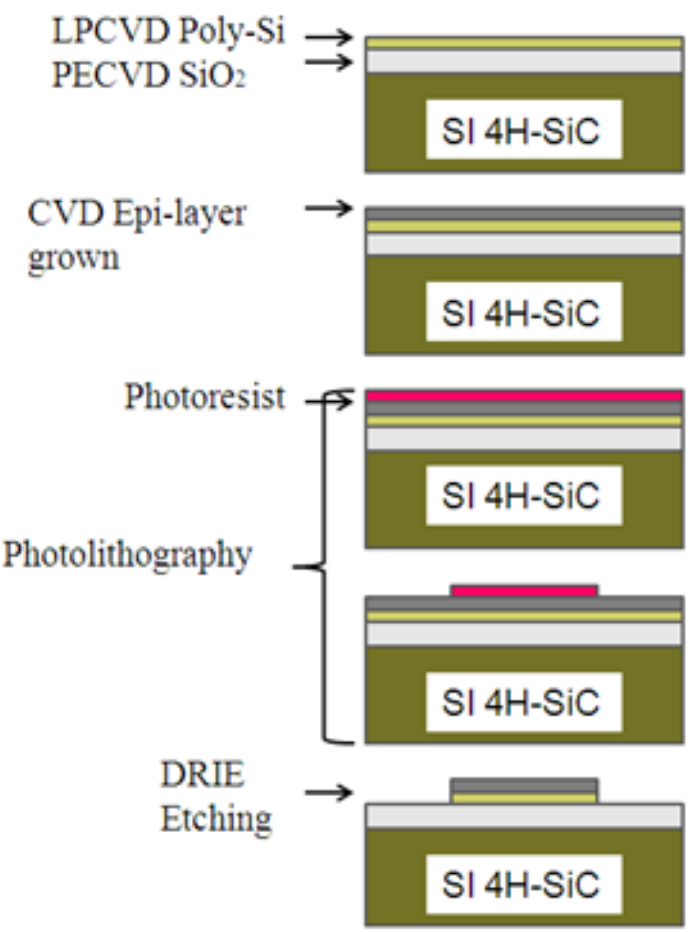

Figure 15: Fabrication process flow of approach $b$ 
The duration of the growth plateau was 1 hour and 40 minutes and yielded a $1.5 \mu \mathrm{m}$ thick film for the antenna structure. The poly 3C-SiC antenna electrode was grown at corresponding pressure, temperature and $\mathrm{Si} / \mathrm{C}$ ratio of 550 Torr, $1250^{\circ} \mathrm{C}$ and 0.35 where the Nitrogen flow was $50 \mathrm{sccm}$.

The film morphology was assessed using optical microscopy and XE-100 atomic force microscopy (AFM). Figure 14(a) shows the AFM images of the polycrystalline SiC films grown on Poly-Si/SiO 2 -coated $\mathrm{SI} 4 \mathrm{H}-\mathrm{SiC}$ substrates with $5 \times 5 \mu \mathrm{m}$ and epilayer photograph, respectively. The surface with uniform agglomerated spherical grains is clearly shown in the AFM image. The rms roughness of the image for a $5 \times 5 \mu \mathrm{m}$ scan is $16 \mathrm{~nm}$. The smooth surface indicates the optimized growth conditions for the polycrystalline 3C-SiC films grown on $\mathrm{SiO}_{2}$-coated $\mathrm{Si}$ substrates. Figure 14 (b) shows the photograph of the CVD grown poly 3C-SiC layer.

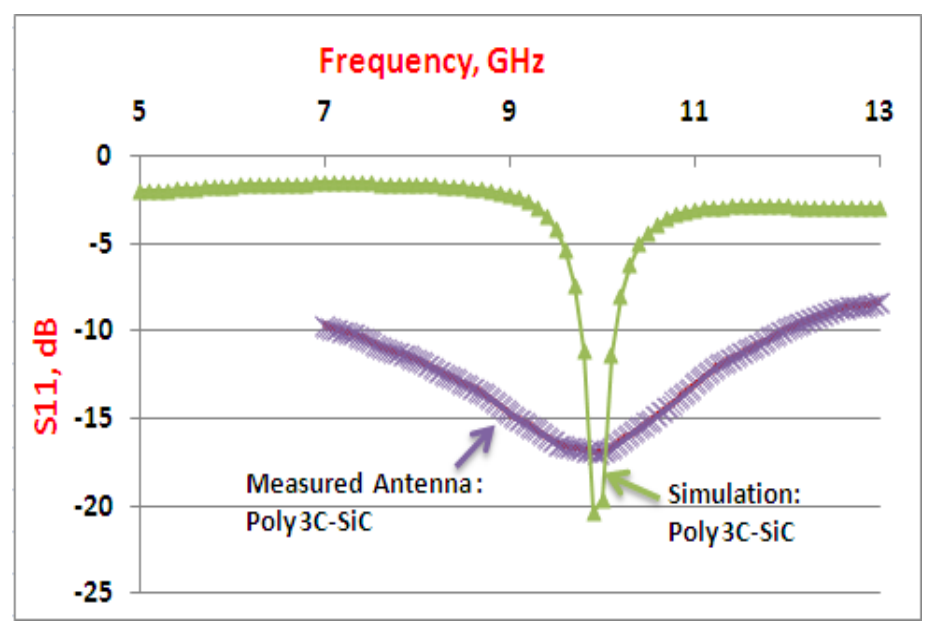

Figure 16: The simulated and measured poly 3C-SiC antenna (approach (b)) responses showing the return loss and resonant frequency. Further optimization is needed to improve the radiation peak but note that the center frequency is at the same value as predicted by simulation [44]

After growing the antenna electrode using CVD, the antenna structure was fabricated by etching back to the substrate using standard photolithography and DRIE etching, the 
fabrication process is shown in Figure 15. After fabrication, the antenna response was measured using Vector Network Analyzer, an HP 8719D VNA. The resonance peak of the measured antenna is comparable to the simulated resonance peak which is shown in Figure 16 (Return loss vs. Frequency). But the overall response of the fabricated antenna is quite lossy. In simulation the doping concentration of Nitrogen was $10^{20} / \mathrm{cm}^{-3}$ for poly 3C-SiC layer with a thickness of $2 \mu \mathrm{m}$. But the fabricated antenna electrode is $1.5 \mu \mathrm{m}$ thick with lower Nitrogen dopant which makes this weak radiation signal. After secondary ion mass spectrometry (SIMS) analysis and antenna simulation, the estimated doping concentration was $\sim 1 \times 10^{19} \mathrm{~cm}^{-3}$ which is lower than the simulated doping concentration. Some figures of this chapter have been reprinted with permission [44].

\subsubsection{Fully Planar Ion Implantation, Approach (c)}

The next research focused on approach (c) which was started by using die cut from the same semi-insulating $4 \mathrm{H}-\mathrm{SiC}$ substrate, patterning it with the simple $8.5 \mathrm{~mm} \times 11.5$ $\mathrm{mm}$ patch antenna. To get heavily doped antenna region (metal like conductivity), other than in-situ doping, ion implantation can also be applied on $\mathrm{SiC}$ material. In principle, ion implantation is ideally suited for introducing dopants in $\mathrm{SiC}$ because of the possibility to accurately control the dopant concentration and the thickness of the implanted region without any chemical or thermodynamic constraints.

The highly resistive semi-insulating substrate was purchased, masked (Nickel mask) and sent out for ion implantation, at a high enough energy to synthesize a degenerate $\mathrm{SiC}$ layer to form the antenna electrode structure. Figure 17 shows the fabrication process flow of structure (b) using ion-implantation. 


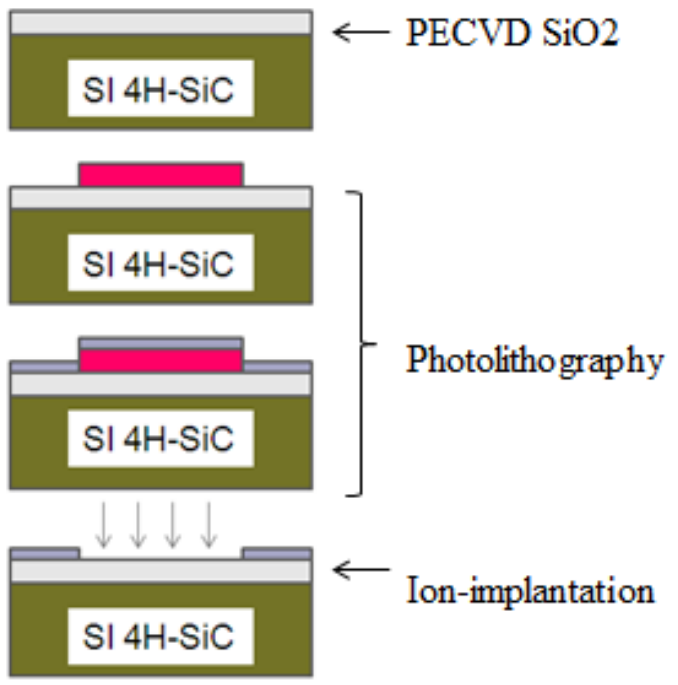

\section{$\mathrm{SI} 4 \mathrm{H}-\mathrm{SiC}$}

$\leftarrow$ Ion-implanted region

Figure 17: Fabrication process flow of approach (c)

Figure 18(a) is the result of simulation for ion implantation doping (Nitrogen doped) profile using SRIM-TRIM simulation software.

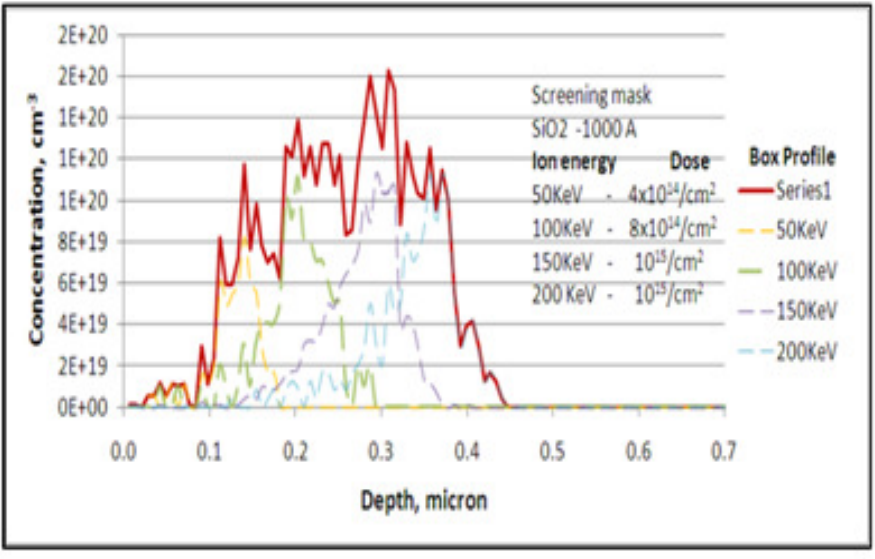

(a)

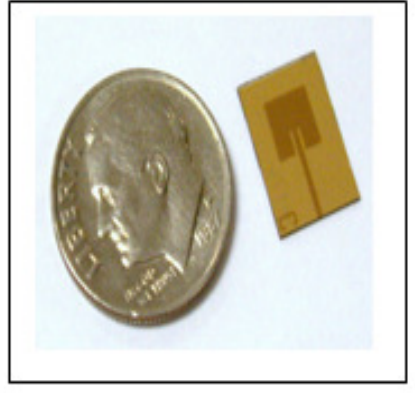

(b)

Figure 18: (a) The simulation for ion implantation on $\mathrm{SiC}$ for different energy levels to get high concentration of doping profile. (b) The fabricated antenna using ionimplantation 
Individual implants are shown in the figure with an overall combined dopant species box profile. The implant energies input into the nitrogen SRIM simulation were: 50, 100, 150 and $200 \mathrm{keV}$. The implant ion doses used to simulate the doping profile were: $4 \times 10^{-}$ ${ }^{14} / \mathrm{cm}^{2}, 8 \times 10^{-14} / \mathrm{cm}^{2}, 10^{15} / \mathrm{cm}^{2}$ and $10^{15} / \mathrm{cm}^{2}$ for the implant energies, respectively. The maximum doping concentration was in the range of $1 \times 10^{20} \mathrm{~cm}^{-3}$ known as heavily doped. The backside of the antenna was also implanted to make a ground plane. After implantation the Ni mask was removed and the fabricated antenna is as shown in Figure 18 (b).

Pre and post implantation characterization was done. Before doing high temperature post implant annealing the surface morphology was evaluated AFM. It has been studied that a major drawback of an implantation process is the generation of damage destroying the crystalline structure of the semiconductor material. The damage can range from point defects caused by single collision cascades at low ion doses to complete amorphization at high enough doses [39]. Therefore, AFM analysis (see Figure 19 (a)) was performed and displayed a surface root mean square roughness, Rq, of $3.48 \mathrm{~nm}$ RMS for a $5 \mu \mathrm{m} \times 5 \mu \mathrm{m}$ scan size, a delta of $1.4 \mathrm{~nm}$ compared to starting surface which had a roughness of $2 \mathrm{~nm}$. After implantation, the current-voltage characteristic was measured using the HP4245 parameter analyzer. Figure 19 (b) shows that the device is very resistive, a small current in the range of nano ampere was observed at voltage of as high as $80 \mathrm{~V}$ with a corresponding resistance was in the range of $\mathrm{G} \Omega$.

Post-implant annealing is required to restore the crystal structure and electrically activate the implanted dopants by positioning them substitutionally in the lattice. The same CVD reactor was also used to anneal ion-implanted $\mathrm{SiC}$ layers. 


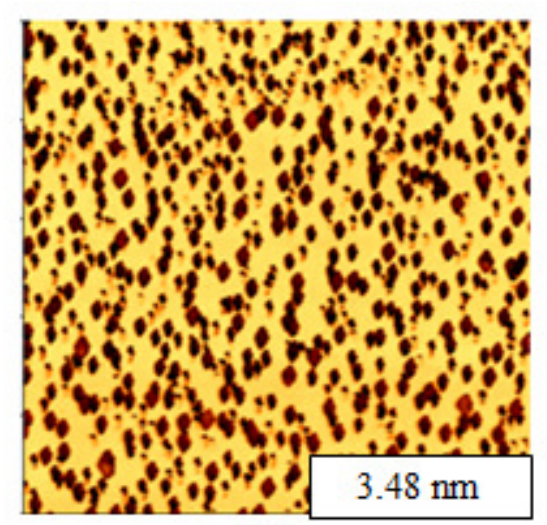

(a)

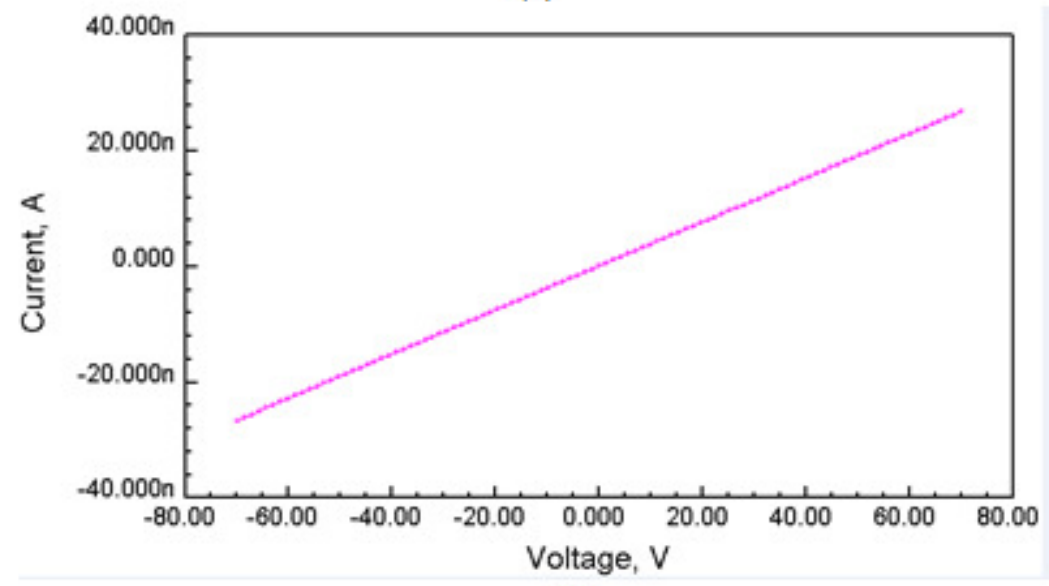

(b)

Figure 19: (a) AFM micrographs of ion implanted surface morphology. AFM data was taken in tapping mode. $5 \mu \mathrm{m} \times 5 \mu \mathrm{m}$ scan area, (b) current-voltage (I-V) characteristics of ion-implanted antenna after implantation showing a highly resistive (G $\Omega$ range resistance) surface

Different annealing approaches are available, one of them is to form a graphite cap on the surface that protects the surface during high-temperature implant annealing, and then the ions are activated to achieve the high doping (conductivity) levels necessary to meet the sensor specifications. So to complete the device processing, the annealing has been performed, at $1400^{\circ} \mathrm{C}, 1550^{\circ} \mathrm{C}, 1600^{\circ} \mathrm{C}$ temperatures in the presence of $20 \mathrm{slm}$ Ar flow for 30 minutes with a graphite cap. 
There was no graphite cap used on the surface at a temperature $1400^{\circ} \mathrm{C}$ because the Si melting point is $1410^{\circ} \mathrm{C}$. Therefore there is little/no chance of sublimation of $\mathrm{Si}$ at this temperature. However, for higher temperatures graphite caps were formed to protect the surface by spinning photoresist following a hard bake for 30 minute in the CVD reactor at $1550^{\circ} \mathrm{C}$ in an $\mathrm{Ar}$ ambient. The graphite cap was removed using an Oxygen Plasma Asher.

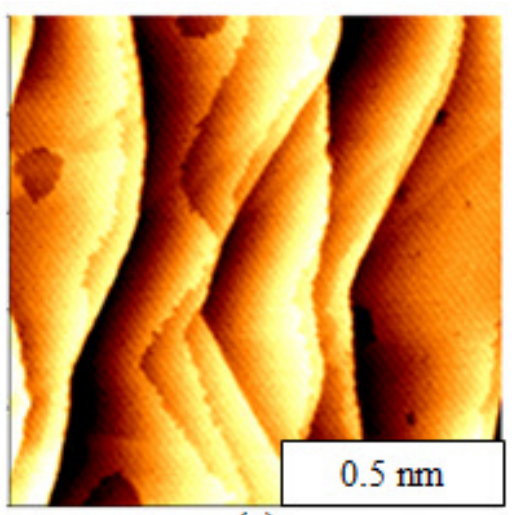

(a)

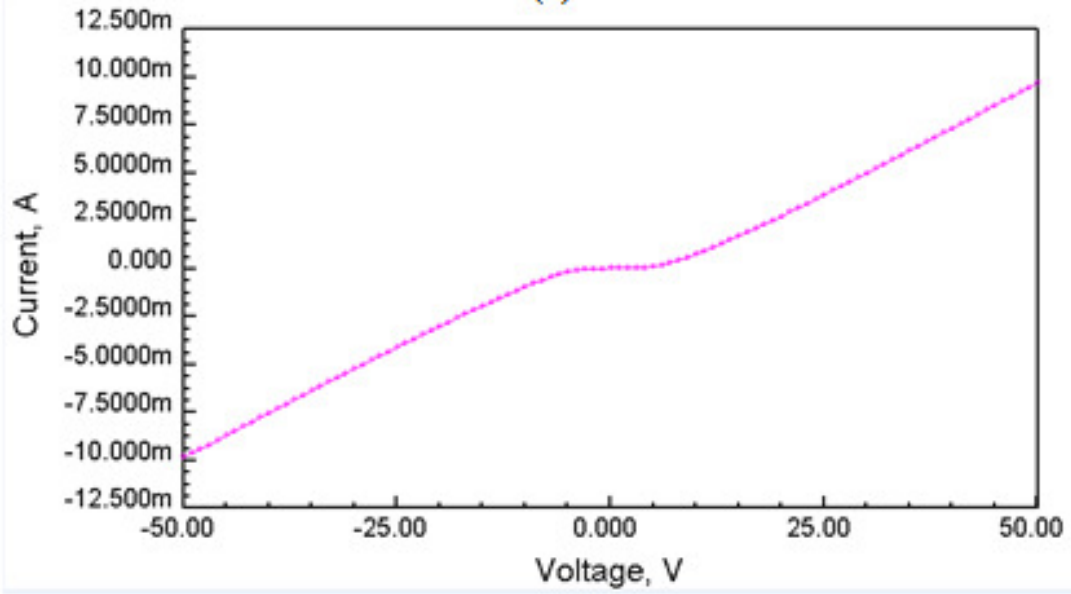

(b)

Figure 20: (a) AFM micrographs of surface morphology after post ion implant annealing. AFM data was taken in tapping mode. $5 \mu \mathrm{m} \times 5 \mu \mathrm{m}$ scan area, (b) current-voltage (I-V) characteristics after post anneal showing less resistive $-500 \Omega-5000 \Omega$ which was $6 / 7$ orders of magnitude lower.

After removing the graphite cap, AFM analysis (see Figure 20(a)) was performed and a surface root mean square roughness, Rq, of $0.5 \mathrm{~nm}$ RMS for a $5 \mu \mathrm{m}$ x $5 \mu \mathrm{m}$ scan size 
was observed. This yields a delta of $2.9 \mathrm{~nm}$ compared to a post implantation roughness of $3.47 \mathrm{~nm}$. So the surface roughness reduces by $2.9 \mathrm{~nm}$ thus indicating an improved surface morphology.

Current-Voltage characterization was also performed in the antenna region of the sensor. The Figure 20 (b) show that lower than milli amperes of current was flowing through the implanted region indicating that the dopants are at least partially activated if not fully activated after the thermal activation anneal. The conductivity was increased by decreasing the resistance in the range of hundreds $(500 \Omega)$ to thousands $(5000 \Omega)$ depending on annealing processes. As a result, post implant annealing improved the surface morphology and the conductivity by reducing 6-7 orders of magnitude of resistance as compare to before annealing.

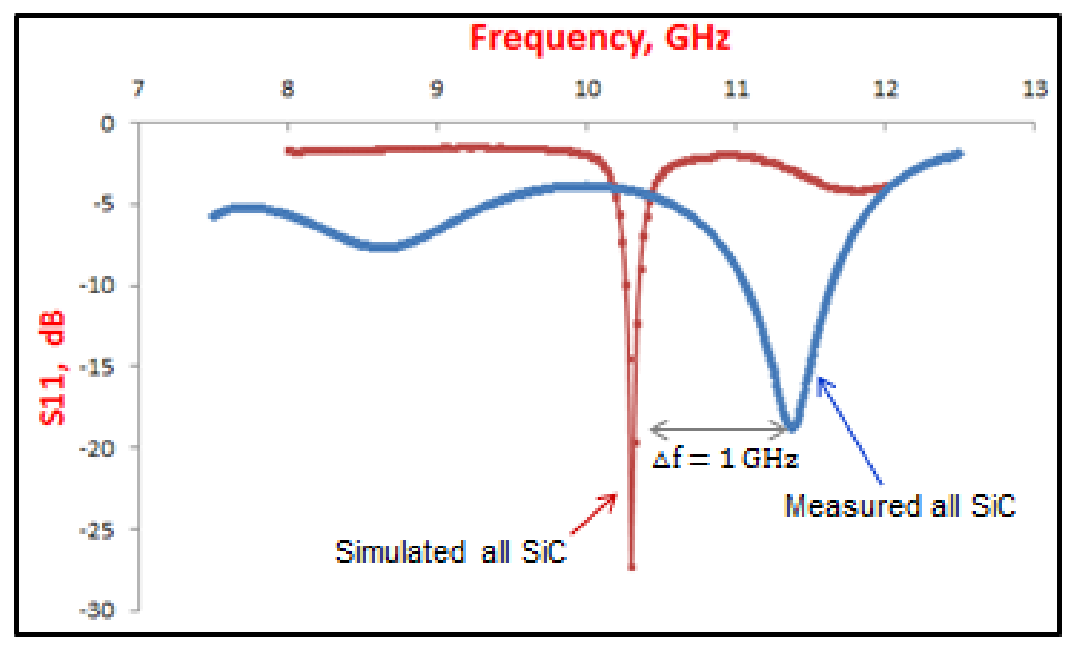

Figure 21: All SiC antenna responses (structure (c)) compared with the Ti/Au antenna response. While the performance of the all-SiC antenna is clearly not optimal it did radiate with a respectable radiation peak that was shifted to higher frequency than the ideal metal-patch (Ti/Au) antenna.

The dopants were partially activated because of the annealing process. The antenna response was measured using the same VNA as discussed in the last section. The all- 
$\mathrm{SiC}$ antenna was directly compared to the $\mathrm{Ti} / \mathrm{Au}$ on $4 \mathrm{H}-\mathrm{SiC}$ antenna (as described in Section 2.2.1). The all- SiC measured antenna resonance peak was comparable to the $\mathrm{SiC}$ based $\mathrm{Ti} / \mathrm{Au}$ (metal) antenna resonance peak, which is shown in Figure 21 (Return loss vs. Frequency ), but the overall response of the all- $\mathrm{SiC}$ fabricated antenna was lossy for glucose sensing purposes. Therefore the $\mathrm{SiC}$ based $\mathrm{Ti} / \mathrm{Au}$ antenna was further evaluated to investigate the glucose sensing mechanism.

\subsection{Results and Discussion}

In this chapter, we have tried to grow heavily nitrogen doped $\mathrm{SiC}$ epilayers to fabricate different approaches of all-SiC antennas. In approach (a), an in-situ n-type doped $4 \mathrm{H}-\mathrm{SiC}$ homoepitaxial layer was grown on an on-axis $4 \mathrm{H}-\mathrm{SiC}$ substrate and the maximum measured doping concentration was $4.2 \times 10^{18} \mathrm{~cm}^{-3}$. From the simulation, it was shown that at least $4 \times 10^{19} \mathrm{~cm}^{-3}$ doing concentration is indeed in need for reasonable radiation to be generated. Therefore, this antenna (approach (a)) has not been fabricated. It is suggested for future work to utilize research funding to purchase costly off-axis semi-insulating $4 \mathrm{H}-\mathrm{SiC}$ substrates to grow on to achieve a lower defect density [37]. The second approach, in-situ nitrogen doped poly-3C-SiC approach, over hundred films have been grown to obtain heavily doped antenna electrode. After fabrication and measurement the antenna response was quite lossy. From SIMS analysis and antenna simulation, the estimated doping concentration was $\sim 1 \times 10^{19} \mathrm{~cm}^{-3}$. The third approach, ion-implantation and post implant activation approach. We have gone through all steps of ion-implantation processes starting from simulation to post implant high temperature annealing. This response was also lossy as compare to Ti/Au or metal patch antenna. 
High temperature annealing is very challenging because - around $1600^{\circ} \mathrm{C}, \mathrm{Si}$ can evaporate causing a rough surface even with the presence of a graphite cap to prevent $\mathrm{Si}$ sublimation. Therefore, the initial results from a fabricated and measured 'all $\mathrm{SiC}$ ' antenna (approach (c)), showed a lossy response basically due to a low doping concentration.

For glucose sensing - it is very important to have an antenna with good resonance peak to perform as a glucose sensor. $\mathrm{SiC}$ based $\mathrm{Ti} / \mathrm{Au}$ antenna has been fabricated and measured resulting in a good response in direct comparison to the simulation data. In the following chapters, we will be mainly focused on biocompatible $\mathrm{SiC}$ based $\mathrm{Ti} / \mathrm{Au}$ antenna for glucose sensing using mimicking blood and pig's blood as an implantable continuous glucose sensor. 


\section{CHAPTER 3}

\section{SIC-BASED ANTENNA AS A GLUCOSE BIOSENSOR}

\subsection{Introduction}

In recent years, considerable progress has been made in developing implantable biosensors/antennas that can continuously monitor different health care indicators in patients, such as the glucose level in a diabetic patient [45]. In particular, the use of radio frequency identification devices (RFIDs) [46] and systems to perform biotelemetry have been reported [47]. It is now possible to implant various wireless sensors inside the human body to monitor numerous bodily functions and transmit health related information outside of the human body for remote monitoring [45]. In this study, we have demonstrated the feasibility of a biocompatible $\mathrm{SiC}$ based implantable sensor, which works using electromagnetic energy propagation principles. This implantable sensor is a promising approach for real-time analysis of continuous blood glucose levels detected by glucose-induced dielectric variations in the patient blood. More specifically, the antenna responds by exhibiting a shift in its resonance frequency as the varying glucose concentration changes the effective complex permittivity of the blood [11]. Being able to detect this glucose variation-induced change in frequency is one of the features of the proposed biocompatible SiC-based sensor. In addition, a biocompatible SiC-based sensor has the potential to remain functional in the body for longer periods of time when 
compared to non-biocompatible sensors. Another advantage of using this SiC-based sensor is that it does not require direct contact with interstitial fluid in order to trigger a necessary chemical reaction to operate. Therefore, in this chapter, a biocompatible SiCbased sensor utilizing a $\mathrm{Ti} / \mathrm{Au}$ low profile radiating patch antenna that was developed is presented.

\subsection{Sensor Operational Principle}

Our long-term goal is to develop a compact implantable device that can continually monitor blood glucose levels. The changes in the glucose level in the medium surrounding the antenna affect the antenna parameters, such as input impedance and reflection coefficient, $S_{11}$. These changes can be used to estimate the patient's plasma glucose level [48-50]. Furthermore, the antenna response can be derived using standard transmission line theory [34].

For a diabetic patient, an increase in glucose causes the glucose transporter process to occur, initializing metabolic oxidation of glucose to produce adenosine triphosphate (ATP) $[11,51]$. The transportation of ionic particles in blood, i.e. $\mathrm{Na}^{+}$and $\mathrm{K}^{+}$are regulated by these ATP through cell membrane or channel. These ionic particles induce a change in electrical impedance and capacitance across the cell membrane. Livshits et al. quantified these changes in the membrane in comparison to glucose concentrations [52]. The sensor's sensitivity depends on the permittivity surrounding the sensor, which affects the sensor's response in terms of electromagnetic radiation frequency shifting. This shifting of frequency is basically related to the corresponding blood glucose levels. Other minerals present in the blood, such as calcium, chloride, potassium and 
magnesium, have been shown to have a minor effect on antenna parameters [53]. Even though in blood sodium and chloride exist in large quantities but only vary from 310 $\mathrm{mg} / \mathrm{dl}$ to $333 \mathrm{mg} / \mathrm{dl}$ and $337 \mathrm{mg} / \mathrm{dl}$ to $372 \mathrm{mg} / \mathrm{dl}$, respectively. But for a diabetic patient, the glucose level varies from $<65 \mathrm{mg} / \mathrm{dl}$ to $400 \mathrm{mg} / \mathrm{dl}$ [54]. Other minerals such as magnesium - 1.8-3.4 mg/dl, potassium - 13.6-21.4 mg/dl, calcium: 8.5-10.5 mg/dl exist in very low quantities in the blood [54]. As a result, it is believed that the performance of the proposed glucose sensor can be evaluated reliably (i.e., has the necessary specificity).

In this study, we used numerical simulation of the antenna response to different blood plasma glucose permittivities and conductivities, and experimental measurements to characterize and evaluate the sensor. In addition, in vitro and in vivo investigations were conducted to monitor the antenna sensor's functionality inside the body's environment to muscle, skin and fatty tissue, (in vitro study) and the compatibility of the sensor's materials to the body for 1 month (in vivo study). First, the in vitro test of the antenna was performed using animal tissue and fat. Secondly, from the simulations, it was found that the best operational position of this implantable sensor is in the fatty tissue region in close proximity to a blood vessel. Therefore, a fat mimicking liquid was prepared and placed in close proximity to the sensor for testing. A mimicking liquid of synthetic body fluid (SBF), which is equivalent to blood plasma, was also made to test for changes in glucose levels. Finally, to determine the antenna response versus glucose levels, with 0 $\mathrm{mg} / \mathrm{dl}$ baseline glucose level, three different amount of glucose were added to the blood mimicking liquid with values ranging from $120 \mathrm{mg} / \mathrm{dl}$ to $530 \mathrm{mg} / \mathrm{dl}$. The detailed design, material properties and in vitro antenna response in air was presented in Chapter 2. This 
chapter elaborates more on the details of the antenna's response in muscle, fatty tissue, and blood.

\subsection{Antenna Imbedded in Animal Muscle and Fatty Tissue}

After fabrication of the SiC-based $\mathrm{Ti} / \mathrm{Au}$ antenna sensor, the antenna's return loss was measured in air. The antenna was then embedded in animal tissue to investigate the correlation of resonance frequency shifts to in the dielectric loading of animal tissue. We selected fresh fat and muscle chicken and cow (beef) purchased at a local supermarket. The permittivity of animal fat $\left(\varepsilon_{\mathrm{r}} \approx 4\right)$ is smaller than the permittivity of muscle $\left(\varepsilon_{\mathrm{r}} \approx 40\right)$, and we were able to observe resonance frequency shifts when comparing animal fat and muscle.

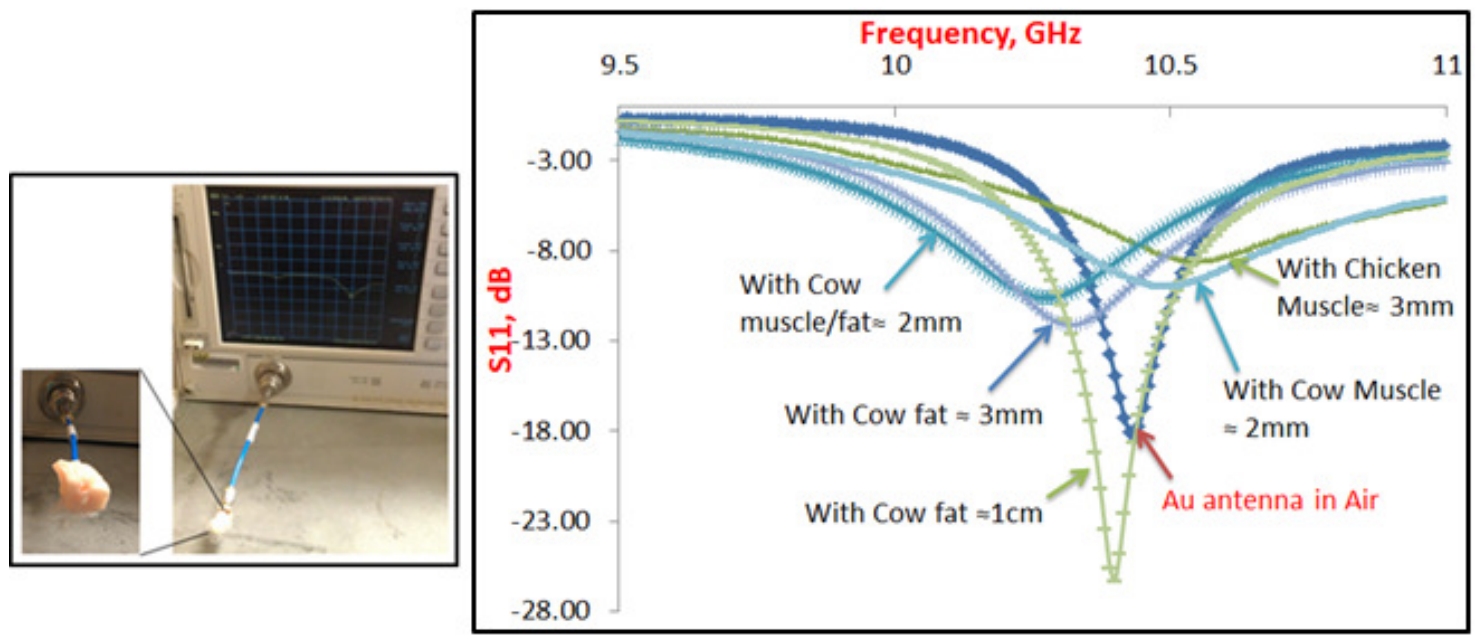

(a)

(b)

Figure 22: (a) Photograph of the experimental setup using a VNA, (b) SiC based Ti/Au antenna resonance frequency shift vs. permittivity changes from fat/muscle (chicken and cow)

The experimental set up and the measured antenna sensor response to fat and muscle in close proximity to the antenna is shown in Figure 22. The antenna response with 
muscle results in a higher resonance frequency as compared to a lower frequency shift with fat. From these performance observations of the fabricated $\mathrm{SiC}$ based Ti/Au antenna sensor in a tissue environment, we were able to confirm shifts in resonance frequency due to permittivity changes. This analogy will be further extended to the changes in permittivity for different glucose levels.

\subsection{Fat and Blood Plasma Mimicking Liquid Preparation}

According to simulations the antenna performed best in the fatty tissue region; therefore, the preparation of fatty tissue mimicking liquid is very important for in vitro testing. The mimicking liquid was developed by mixing oil and DBGE (Di Butyl Glycol Ether). Following the initial tests, an optimized recipe for fatty tissue-mimicking liquid for $10 \mathrm{GHz}$ was formulated, which consisted of $10.2 \%$ oil mixed with $89.8 \%$ DGBE. This fatty tissue-mimicking liquid was based on measured properties of human fatty tissue (fat, $\varepsilon_{\mathrm{r}} \approx 5$ ) taken from literature [55-57].

In order to measure the blood glucose level, a synthetic body fluid (SBF), or acellular human blood plasma mimicking liquid, was prepared. Table 2 shows the ingredients / chemical composition of the human blood mimicking solution. This solution was prepared by dissolving the appropriate quantities of the chemicals in de-ionized water. The reagents of Table I were added one-by-one after the previous reagent was completely dissolved in $700 \mathrm{ml}$ of $\mathrm{DI}$ water. This solution was then titrated with $1 \mathrm{M} \mathrm{HCl}$ for $\mathrm{pH}$ (7.4) adjustment. During the titration process, the solution was also continuously diluted with consecutive additions of de-ionized water to make the final volume equal to 1 liter [5859]. The prepared blood plasma solutions can be stored at $5^{0} \mathrm{C}$ for a month without degradation. 
The corresponding amount (in grams) of D-glucose was added to the blood mimicking liquid in order to make three different blood glucose levels for this study, which is shown in Table 3. The glucose levels were measured using a conventional glucometer (TRUEtrack, Blood glucose monitoring system [60].

Table 2: Recipe for blood plasma mimicking liquid

\begin{tabular}{|c|c|c|}
\hline Order & Reagent & Amount (gpl) \\
\hline 1 & $\mathrm{NaCl}(99.5 \%)$ & 6.567 \\
\hline 2 & $\mathrm{NaHO}_{3}(99.5 \%)$ & 2.268 \\
\hline 3 & $\mathrm{Na}_{2} \mathrm{SO}_{4}$ & 0.368 \\
\hline 4 & $\mathrm{Na}_{2} \mathrm{H}-\mathrm{PO}_{4} .2 \mathrm{H}_{2} \mathrm{O}(99.5 \%)$ & 0.178 \\
\hline 5 & $\mathrm{KCl}(99.0 \%)$ & 0.373 \\
\hline 6 & $\mathrm{MgC}_{12} \cdot 6 \mathrm{H}_{2} \mathrm{O}(98 \%)$ & 0.305 \\
\hline 7 & $\mathrm{CaC}_{12} .2 \mathrm{H}_{2} \mathrm{O}(99 \%)$ & 6.057 \\
\hline 8 & $\left(\mathrm{CH}_{2} \mathrm{OH}\right)_{3} \mathrm{CNH}_{2}(99.2 \%)$ & 0.071 \\
\hline
\end{tabular}

Table 3: D-glucose weight vs. glucose concentration*

\begin{tabular}{|c|c|}
\hline $\begin{array}{c}\text { Glucose concentration } \\
(\mathrm{mg} / \mathrm{dl})\end{array}$ & D-Glucose (grms) \\
\hline 0 & 0 \\
\hline 120 & 0.021 \\
\hline 270 & 0.039 \\
\hline 530 & 0.082 \\
\hline
\end{tabular}

* within $50 \mathrm{ml}$ volume of blood mimicking liquid

\subsubsection{Mimicking Liquid's Permittivity}

The in vitro measurement of the dielectric permittivity of the fatty tissue- equivalent liquid was performed by using a HP 85070A dielectric probe kit. This kit was used in conjunction with a HP 8719D vector network analyzer (VNA) to measure the complex reflection coefficient, $S_{11}$, of the material under test. The reflection coefficient was then converted into the complex permittivity of the liquid. Figure 3.2 shows the measured real 
part of the permittivity for fat, $\varepsilon_{\mathrm{r}} \approx 5$ at $10 \mathrm{GHz}$, which is very close to reports from literature $[50,57,61]$. The conductivity was calculated from the imaginary part of the permittivity and the calculated value was $\sigma \approx 0.51 \mathrm{~S} / \mathrm{m}$ at $10 \mathrm{GHz}$. Similarly the permittivity of the different glucose levels established in the blood mimicking samples was measured as shown in Figure 23. The corresponding conductivities were also calculated. We have observed similar trends for increases glucose concentration of the blood solution corresponding to decreases in the permittivity and conductivity of the solution, as reported by (E. Topsakal [53] and Venkataraman [14]). As expected, the permittivity varies with glucose levels. In addition, the corresponding conductivities were calculated from the measured imaginary part of the permittivity as $18 \mathrm{~S} / \mathrm{m}, 15 \mathrm{~S} / \mathrm{m}$ and 13 $\mathrm{S} / \mathrm{m}$ for the blood mimicking liquids exhibiting $120 \mathrm{mg} / \mathrm{dl}, 270 \mathrm{mg} / \mathrm{dl}$, and $530 \mathrm{mg} / \mathrm{dl}$ glucose concentrations, respectively.

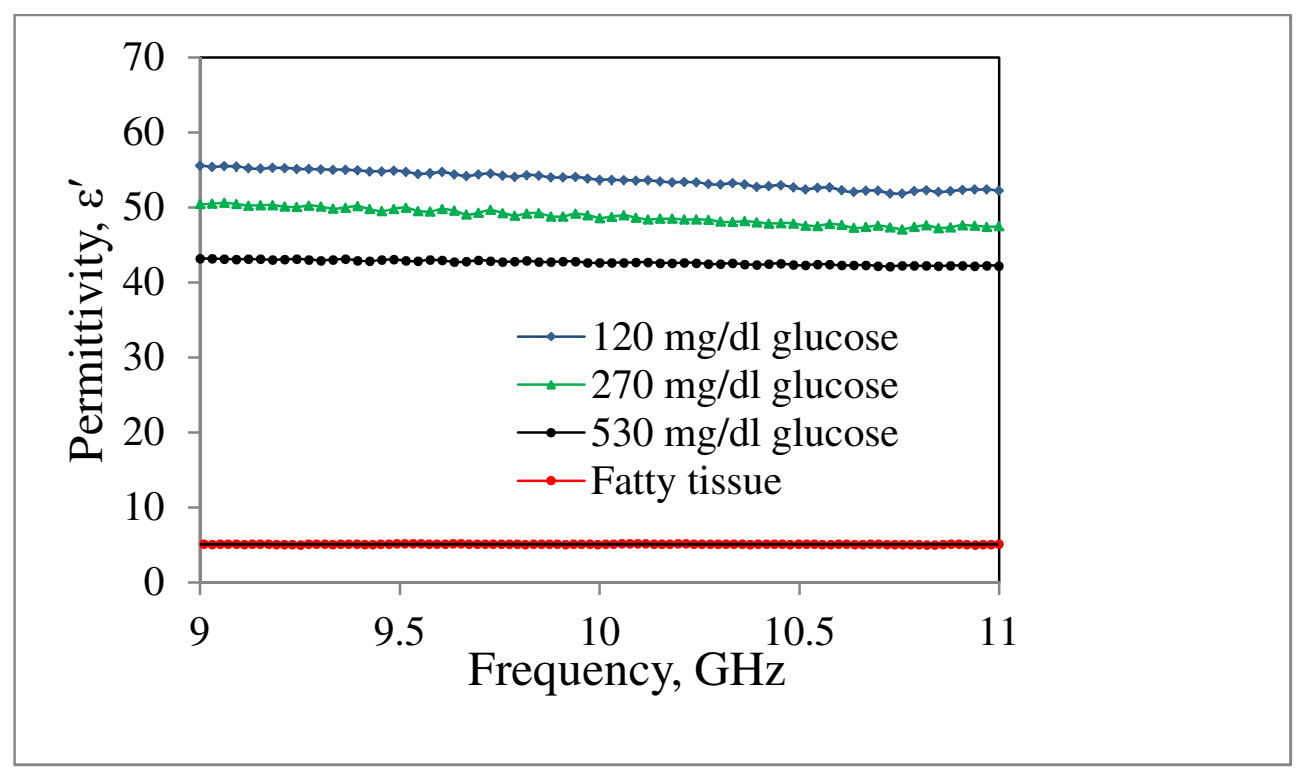

Figure 23: Measured relative permittivity of fat and different glucose levels of blood mimicking liquid vs. frequency 


\subsubsection{Experimental Setup for Blood Glucose Monitoring}

After preparing the fatty tissue and blood plasma mimicking liquids, the antenna sensor was tested using the HP8719D VNA. Ideally, the sensor should be implanted in the fatty tissue region and the electromagnetic wave will propagate through the blood vessel/interstitial body fluid and the resulting $S_{11}$ is used to estimate the plasma glucose level. A similar experimental set up was made by placing the antenna sensor in close proximity to fatty tissue upon which a blood plasma layer was used to probe the system response to varying glucose concentrations. The experimental setup is shown in Figure 24.

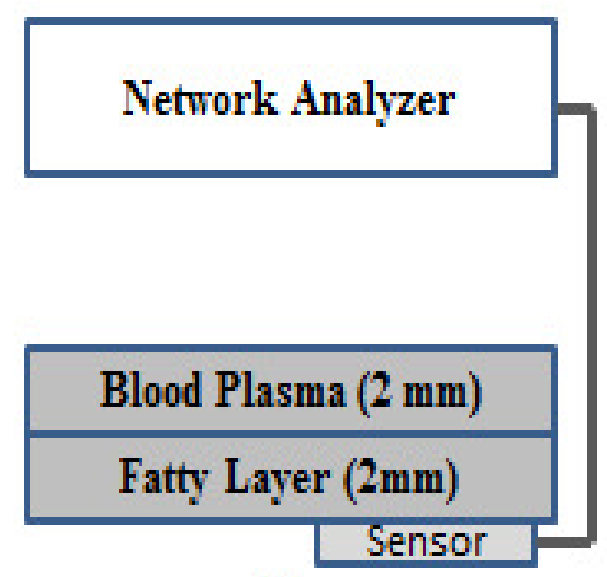

(a)

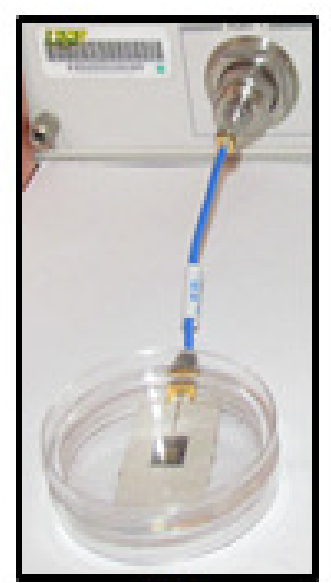

(b)

Figure 24: Setup for measuring the blood plasma glucose level (a) schematic diagram and; (b) photograph of the experiment

First blood mimicking fluid was synthesized without any glucose to take a baseline reading from the sensor. Once this baseline response was measured different amounts of glucose were added to the blood mimicking liquid. Three samples were prepared and their glucose levels measured using the same conventional blood glucose monitoring system. The measured values were $120 \mathrm{mg} / \mathrm{dl}, 270 \mathrm{mg} / \mathrm{dl}$ and $530 \mathrm{mg} / \mathrm{dl}$, respectively. 
The antenna sensor was tested using these blood samples, where the synthetic fatty tissue layer, directly in contact with the sensor, was unchanged.

The thicknesses of the fatty tissue and blood mimicking liquid layers were $2 \mathrm{~mm}$ each and the volume was $5 \mathrm{ml}$ each. For a near-field radiation measurement, the antenna resonance frequency was observed to shift according to changes in the glucose level in the blood. The experimental performance of the sensor is shown in Figure 24.

\subsection{Results and Discussion}

Figure 25 shows the measured return loss, $S_{11}$, for the blood mimicking samples versus glucose concentration: (a) $0 \mathrm{mg} / \mathrm{dl}$ (no glucose added), (b) $120 \mathrm{mg} / \mathrm{dl}$, (c) 270 $\mathrm{mg} / \mathrm{dl}$, (d) $530 \mathrm{mg} / \mathrm{dl}$. The inset shows the simulated return loss from the blood mimicking samples.

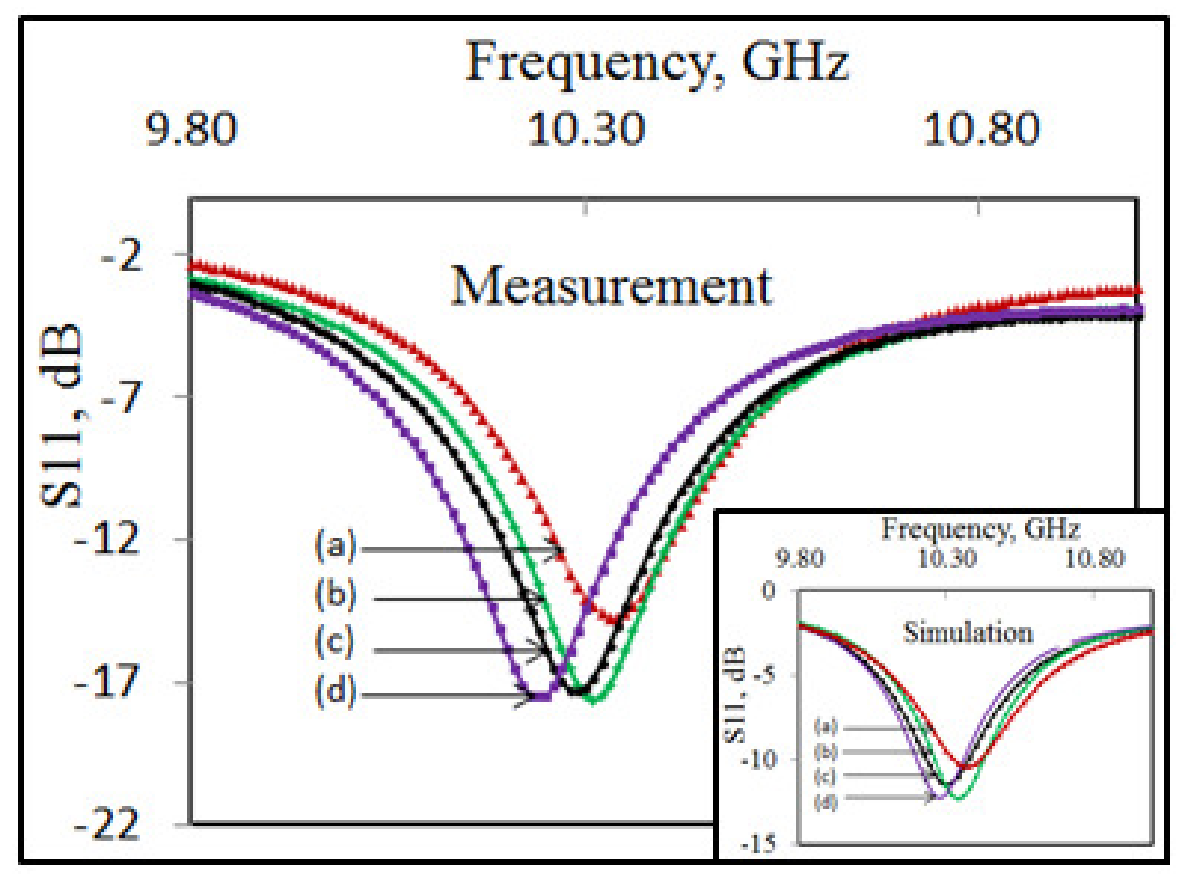

Figure 25: Measured and simulated (inset) $S_{11}$ response of the antenna as a function of glucose concentration within the blood mimicking solution. (a) $0 \mathrm{mg} / \mathrm{dl}$ (no glucose added), (b) $120 \mathrm{mg} / \mathrm{dl}$, (c) $270 \mathrm{mg} / \mathrm{dl}$, (d) $530 \mathrm{mg} / \mathrm{dl}$ 
Ansoft HFSS software was used to simulate the glucose response using the measured permittivity of blood mimicking liquid. For both of the measured and simulated data the resonance frequency was observed to shift to lower frequencies as a function of increasing glucose levels.

The total shift in the resonance frequency of the blood glucose sensor was $40 \mathrm{MHz}$, while the simulated change was $50 \mathrm{MHz}$. Both measured and simulated results were obtained for the same synthetic fat/blood layer sample volume and thickness. We found good agreement with simulated and measured resonance frequency values providing strong evidence that the sensing mechanism can indeed be correlated to a change in medium permittivity (varying glucose level) when in close proximity to the sensor.

The antenna sensor response was measured at multiple glucose levels in order to assess the sensor linearity. The in vitro sensitivity of the sensor was determined by first establishing a baseline sensor value (no addition of glucose in the blood plasma). After stabilization of the background level, increments of glucose were added to the blood mimicking solution to evaluate the sensor response. The in vitro sensor performance demonstrated that the sensor showed a dose-dependent response to glucose concentration from $120 \mathrm{mg} / \mathrm{dl}$ to $530 \mathrm{mg} / \mathrm{dl}$, which is shown in Figure 25.

After getting promising glucose sensing results using blood mimicking liquids, we demonstrated the sensor's performance using pig blood. Prior to pig blood use for this research, a veterinary approval was given by the University of South Florida, Institutional Animal Care \& Use Committee (IACUC). Standard sterile plastic vacutainer blood collection tubes with a closure (the draw of a predetermined volume of blood was done by evacuating to create a vacuum inside the tube) were used in this research to collect and 
preserve the pig's blood. To avoid fast coagulation of the pig blood, EDTA (ethylenediaminetetraacetic acid) was used and the blood was stored at $4^{\circ} \mathrm{C}$ for 3 days. Initially the pig blood had a glucose level of $67 \mathrm{mg} / \mathrm{dl}$. Three additional samples were prepared with glucose levels of $120 \mathrm{mg} / \mathrm{dl}, 270 \mathrm{mg} / \mathrm{dl}$ and $490 \mathrm{mg} / \mathrm{dl}$ by adding Dglucose to the blood. Similar to the glucose sensing experiment using fat and blood mimicking liquids, we demonstrated glucose sensing using pig blood. The sensor response is shown in Figure 26.

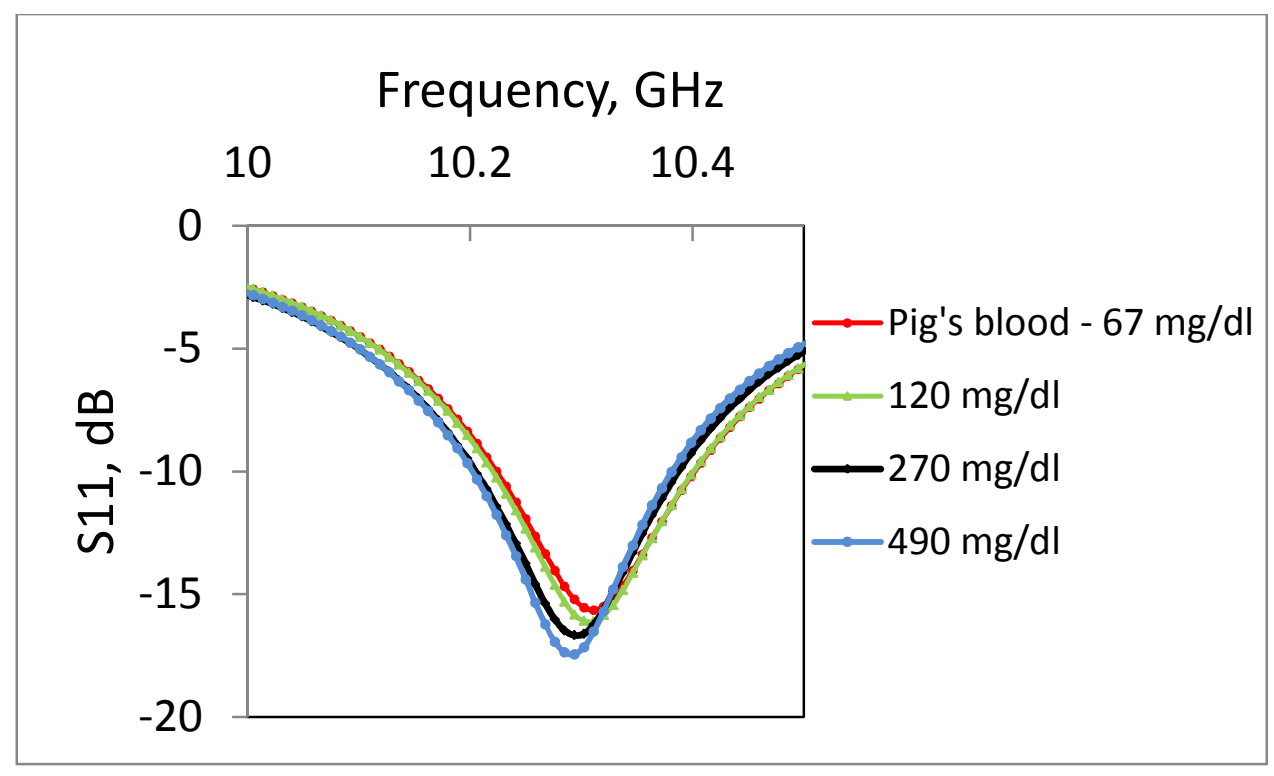

Figure 26: Measured $S_{11}$ response of the antenna to pig blood as a function of glucose concentration for (a) $67 \mathrm{mg} / \mathrm{dl}$, (b) $120 \mathrm{mg} / \mathrm{dl}$, (c) $270 \mathrm{mg} / \mathrm{dl}$, (d) $490 \mathrm{mg} / \mathrm{d}$, respectively. Note the frequency shift to lower frequencies as the glucose level increases, a trend identical to the blood-mimicking response

The dependence of glucose concentration on resonance frequency observed with pig blood followed the same trend as the blood-mimicking experiment discussed earlier. The observed shift in frequency was $62 \mathrm{kHz}$ per $\mathrm{mg} / \mathrm{dl}$ of glucose versus $97 \mathrm{kHz}$ per mg/dl for the pig blood versus blood mimicking fluid experiments, respectively. In addition it was noted that the magnitude of $\mathrm{S}_{11}$ decreased with increasing pig blood glucose concentration 
by $-3 \mathrm{~dB}$ but the signal to noise ratio was excellent therefore indicating that this is not a serious issue.

In Figure 27 it has been shown that the sensor performance was linear with the frequency shift being a direct function of glucose concentration. For the blood mimicking liquid, the total frequency shift $(\Delta f)$ was $40 \mathrm{MHz}$ for a glucose level of $120-530 \mathrm{mg} / \mathrm{dl}$. It has also been shown that there is a $97 \mathrm{KHz}$ resonance frequency shift per $1 \mathrm{mg} / \mathrm{dl}$ $(\Delta f / \Delta S)$ of liquid, as in Figure 25. Similarly for pig blood, the total frequency shift $(\Delta f)$ was $26 \mathrm{MHz}$ over $67-490 \mathrm{mg} / \mathrm{dl}$ of glucose concentrations. It also showed a resonance frequency shift of $62 \mathrm{KHz}$ per $1 \mathrm{mg} / \mathrm{dl}(\Delta f / \Delta S)$ of pig's blood (Figure 26).

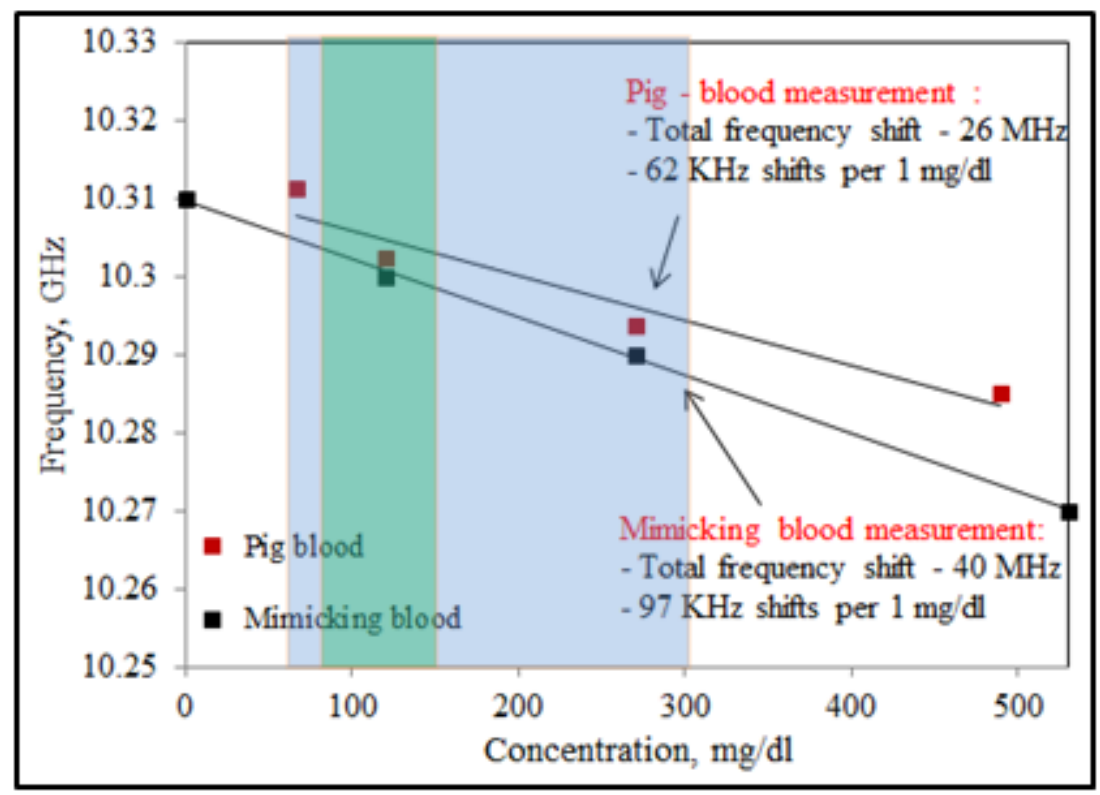

Figure 27: Measured relative resonance frequency shift dependence on glucose concentration. These data were estimated from Figure 25 and Figure 26. This shows that the sensor performance is a linear approximation with the glucose concentration as indicated. Normal (green shaded) to critical (blue) care patient glucose levels are shaded for ease of references [American Diabetes Association] 
Here,

Total Frequency shift, $(\Delta f)=($ Resonance frequency at mimimum glucose level -Resonce frequency at maximum glucose level)

Frequency shifts per glucose concentration $=\Delta f / \Delta S=($ Total Frequency shift $) /$ (Difference of Maximum and Minmum glucose concentrations)

From these results, it is observed that the antenna response to blood mimicking liquid and pig blood have similar trends of frequency shifting - the resonance frequency decreases with an increase of glucose levels. For a non-diabetic healthy person, the American Diabetes Association recommends a fasting plasma glucose level of 70-130 $\mathrm{mg} / \mathrm{dl}$ which is shown in Figure 27 (in green shaded area). Below 60-65 mg/dl is known as hypoglycemia and above $240 \mathrm{mg} / \mathrm{dl}$ is known as hyperglycemia (shown in blue shaded area). The two shaded areas of Figure 27 is the region where the $\mathrm{SiC}$ based sensor covers the normal to critical care application.

In this chapter we reported an in vitro study of the performance of a biocompatible SiC-based glucose sensor. The sensor was designed to determine the glucose level human blood and was tested in vitro using blood mimicking liquid as well as pig blood using an electromagnetic signal analysis. The antenna sensor was able to determine glucose concentration by observing a well-defined shift in the resonance frequency, and this was accomplished without any direct sensor contact with the blood. The results show clear response linearity and promising usefulness for a biocompatible material- SiC-based sensor for long-term, real-time continuous glucose monitoring for diabetic patients. 


\section{CHAPTER 4}

\section{IN VIVO INVESTIGATION OF ANTENNA MATERIALS}

\subsection{Introduction}

A major obstacle of in vivo subcutaneous implantable glucose sensors and other biosensors is the loss of functionality after a relatively short period of time. The result of a biological response to implanted biomaterials in mammals is a complex series of events that involves many biochemical pathways. Implantation of biosensors into tissue, which are foreign objects, represent an acute form of tissue injury. The tissue responds to this injury in a two-step process; the first step is inflammation, and the second step is wound healing. Extensive tissue destruction in an uncontrolled manner starts the inflammatory process and ultimately induces the tissue fibrosis. This chapter elaborates on this twostep process and, based on this knowledge, evaluates the materials used in the development of the proposed $\mathrm{SiC}$ antenna sensor.

After fabrication of different SiC-based antennas, an in vivo experiment for foreign body response to subcutaneously-implanted devices has been conducted using a pig/swine animal model. This data will complement the in vitro biocompatibility study of the different poly types of $\mathrm{SiC}$ that was presented in Chapter 1 Section 3. For the in vivo biocompatibility study, different surface modifications and surface coatings were used. In the following sections, details of the foreign material/living cell interaction, biofouling, 
and histological tissue analysis will be presented. In addition, details of surface modifications employed to inhibit protein adsorption, biocompatible material coatings, and in vivo implantation procedures will be discussed.

\subsection{Foreign Material and Living Cell Surface Interaction}

A number of materials, including SiC, Titanium (Ti), and gold (Au), can be used for biological system devices. These materials, known as biomaterials, play an important role in the integration of bio devices into point-of-care and advanced health care systems. A biomaterial can be defined as a material with a favorable biological response when used in treating or replacing tissue, organs, or bodily functions with bio-devices [62]. Simply - 'biomaterial is any matter, surface, or construct that interacts with biological systems' [63]. It is very important to understand the mechanisms and dynamics of these biological interactions in order to investigate the integration of devices into biological systems. Furthermore, to develop novel biosensors and biomedical devices, a fundamental understanding of the molecular interactions between bio-molecules and biomaterial surfaces is needed. There are a number of issues associated with the use of biomaterials that come in direct contact with biological systems, inclusive of tissue, skin, and blood, for example. Unwanted protein adsorption is one of the significant problems associated with all material surfaces exposed to solutions containing biological samples. For example, shortly after implantation, fibrinogen and other proteins can bind to the device surface causing dysfunctionality, a process referred to as biofouling. This is one of the major problems affecting advances in the biological sciences, including biosensors. The potential impact of non-specific and specific protein adsorption has inspired 
multidisciplinary studies focused on understanding the mechanism of protein adsorption and developing strategies for surface modification aimed at minimizing biofouling [64][68].

Macrophages, a type of white blood cell which promotes immune system regulation and would healing, can bind to receptors on these proteins and work with lymphocytes ( $\mathrm{T}$ cells and B cells in the body) to destroy infected cells and coordinate the overall immune defense system. On the other hand $\mathrm{T}$ cells, in response to tissue injury or fibrinogen attack, secrete proteins called cytokines. These cytokines may cause surrounding immune system cells to thrive or die, or activate non-immune system tissue growth. This non-immune system tissue growth is known as fibrosis, which is the formation of scar tissue with chronic inflammation. Upon tissue injury, myofibroblasts, a form of connective tissue cells, synthesize extracellular matrix proteins, including collagen. After secretion into the extracellular space, collagen becomes cross-linked. As cross-linked collagen and other extracellular matrix proteins mature, it gradually leads to the formation of a hypocellular dense fibrous capsule, that becomes impermeable or hypopermeable to many compounds [69] used for treatment. When these dense fibrous capsules are formed and bind to a device surface, the device/sensor loses functionality which is, in part, a consequence of inflammation and fibrosis resulting from possible reactions between the tissue and implant surface [70]-[72].

For an implanted synthetic material/biomaterial, the biological response is difficult to explain and generally overcome. Ratner et al. [73] illustrates collagen capsule evolution in the presence of a foreign body (Figure 28). This work looks at reactions for various sensor surfaces as presented in Chapter 4 Section 3. 


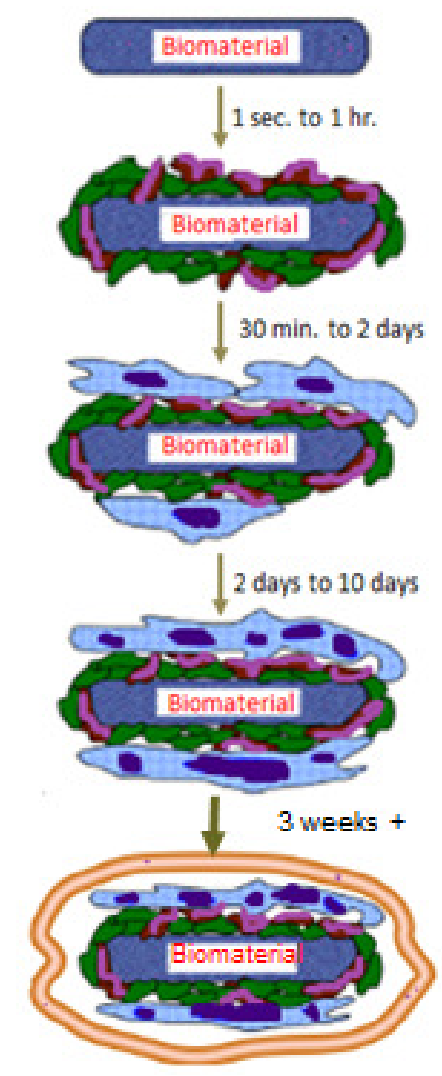

Figure 28: The foreign body reaction illustration by higher organisms to an implanted synthetic material/biomaterial

\subsubsection{Protein Adsorption to Biologically Relevant Surfaces}

As stated earlier upon implantation of an artificial/bio material, protein adsorption is the first step of wound healing process. Linear copolymers of 20 different L-amino acids, known as protein, are at the center of life in this process. They conduct different types of interactions such as protein-protein interactions, protein-nucleic acid interactions, and protein-surface interactions. A definite unfavorable interaction of proteins with the biomaterial surface plays a major role in dictating the performance of that particular system. The most general interaction is the non-covalent interactions between biomolecules and molecules present at the cell surface. A strong, but non-covalent bond, can also occur between two macromolecules with a unique combination of interactions, such 
as ionic, steric, and directional bonds. For specific interactions, the binding energy depends strongly on the local geometry and chemistry [74], and the binding site's affinity and uniqueness are the two major characters. A specific adsorption of proteins to an artificial biomaterial surface can also be utilized in the development of protein sensors for the detection of proteins.

A key event when biomaterial surfaces come in contact with a biological environment is the non-specific adsorption of proteins, which is a complex event that is difficult to illustrate, and often leads to the failure or loss of activity of the sensor or biomaterial. The process of non-specific adsorption of protein is governed by: protein properties (structure, size, and distribution of charge and polarity); the tested biomaterial surface properties (charge, roughness, and state of surface energy); the environmental conditions ( $\mathrm{pH}$, ionic strength, and temperature); and the adsorption process kinetics [75]. Therefore, the prevention of non-specific adsorption of proteins will alter the wound healing response that leads to the attraction of giant foreign body cells and the fibrous capsule formation via cellular mechanisms [76].

In our in vivo study, among different antenna surface coatings, we also studied the inhibition of non-specific protein adsorption on the antenna sensor's surface for extending the sensor's operational life time. The anticipated result is the inhibition of non-specific adsorption of proteins to the surface which improves the biocompatibility of biomedical implants, which is discussed next. 


\subsubsection{Surface Modification}

Surface modification is important to prevent non-specific protein adsorption There are two main aspects of biomaterial properties, bulk properties and surface properties, which are important for applications in different fields. Bulk properties primarily include mechanical, thermal, and magnetic characteristics, while typical surface properties include surface roughness, surface chemistry, surface charge distribution, and interfacial free energy. One of the first steps to implementing an implantable device is matching the bulk properties of an ideal biomaterial to a specific application. As stated earlier, after implantation the biomedical devices elicit a host response mechanism and tend to lose their functionality when the biomaterial surface is exposed to a biological environment. Therefore, surface modification of a biomaterial maybe necessary to improve the biocompatibility of that material, while retaining the desired bulk properties. In this research, among many surface properties, we will focus on one of the surface modifications aimed at preventing non-specific protein adsorption. To render the biologically relevant surfaces protein repellent, a variety of approaches have been developed, they are discussed in detail in the following section, 4.2.3.

\subsubsection{Protein Repellent/Nonfouling Coating Material}

One of the major factors determining the biocompatibility of any biomedical device is surface biofouling. Advancements for non-biofouling materials are still being researched to increase the biocompatibility of implantable devices [77]. To restrict nonspecific protein adsorption efficiently, there are a limited number of such materials available. More recently, four molecular level characteristics have been presented to 
inhibit protein adsorption: first the material should be hydrophilic; second the material should contain hydrogen bond acceptors; third the material should not contain hydrogen bond donors; and, fourth the material's overall electrical charge should be neutral [78]. For non-biofouling coatings that tend to promote the biocompatibility of biomaterials, there are generally two categories: 1) biomimetic materials, including phosphorylcholine (PC), bovine serum albumin (BSA), and other proteins; and 2) synthetic polymers, such as oligo and poly ethylene glycol (OEG/PEG), acrylates and other hydrophilic synthetic polymers.

As a non-biofouling surface PEG is generally accepted as one of the most biocompatible polymers, and has been the subject of immense interest in the preparation of non-biofouling surfaces [79]. Deposition of oligo/poly(ethyleneglycol) (OEG/PEG) chains on a biomaterial surface has already been demonstrated to decrease the biological fouling [80]. In our research, this non-biofouling surface, PEG, was deposited on a SiCbased Ti/Au antenna surface. The following section will focus mainly on the chemical and physical properties of PEG, which contribute to its ability to resist proteins.

\subsubsection{Poly(ethyleneglycol) (PEG) Properties and Functionalization}

PEG, often referred to as poly-ethylene oxide (PEO), is soluble in water and most organic solvents. It is a linear, or branched, neutral polyether (Figure 29) available in a variety of molecular weights. As stated earlier despite its apparent simplicity, PEG has been found to be a highly effective synthetic polymer in reducing non-specific protein adsorption. 


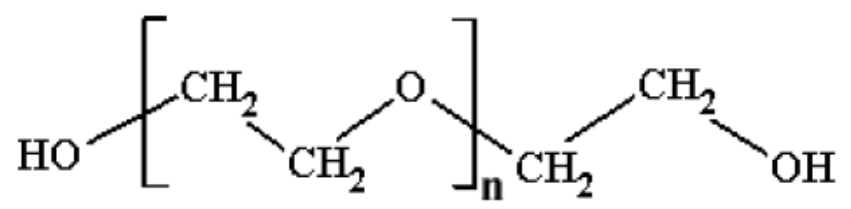

Figure 29: Molecular structure of poly ethylene glycol (PEG)

A lot of work has been done to explore PEG's properties for different biological applications [80]. However, in this section we will briefly discuss PEG's ability to impart non-biofouling character to the biomaterial surfaces and the mechanism of nonbiofouling PEG surfaces. Theories on the mechanism of biofouling resistance by PEG is divided into two opposing schools of thought: 1) the physical view, which involves physical interactions; and 2) the chemical view, involving chemico-molecular interactions [81].

The physical view of fouling resistance is important. Alexander-De Gennes' theory of polymer brushes, one of the most successful theories in the history of polymer physics, is the physical view that biofouling resistance is mainly based on the steric effect [82-85]. Based on this theory, Andrade and Jeon $[86,87]$ were the first to attempt and model protein resistant properties of PEO-coated surfaces. According to this model, the inertness of PEG chains can be explained in terms of steric repulsion, generated by the compression of a tethered PEO layer, as the protein approaches the surface. The dehydration and confinement of the highly flexible, hydrated PEO chains cause this steric repulsion. Additionally, according to this model, long chain length and a high surface density of PEO is necessary for optimal protein resistance. This model is valid only for very long PEO chains $(n>200)$ considering polymer conformation of the brush regime. Another model, known as the single chain mean field theory (SCMF), fully accounts for 
the chain configuration, temperature, surface density of the polymer, and composition of the sample [88-89]. The SCMF model states that the polymer chain length has a less dominant effect, while the surface grafting density of the polymer is a more impactful parameter.

According to Halperin's model, which is the latest milestone in the physical view of biofouling resistance, the effective potential experienced by a protein approaching a polymer coated surface (in brush conformation) results from the sum of: (1) a purely attractive interaction between the protein and the bare surface and (2) a purely repulsive interaction between the swollen polymer brush and the protein [89]. The sum of these two potentials gives rise to an effective interaction that can also be tuned. This model explains the interplay of the thickness of the brush layer, the size of the incoming protein, and the surface grafting density of the polymer chains. Therefore, according to all models described above, regarding the physical view of biofouling resistance, the steric repulsion is the key phenomenon behind protein repellency of PEG grafted surfaces. However, these models depict water as spherical, neutral particles and also do not account for interfacial chemistry and molecular structure. These aspects are addressed in the chemical view of biofouling resistance.

The chemical view of biofouling resistance is also important. According to the physical view, water was considered as a non-interacting molecule but, in practice, water plays a significant role in imparting protein resistance to PEG tethered surfaces. The PEG molecule interacts with the surrounding condensed phase through hydrogen bonding, and it is the basis of the high solubility of PEG in water. The chemical view of biofouling resistance was proposed by Besseling et al. [90-91] using hydration force theory. This is 
the force between surfaces and the fluids including its application to water at interfaces, which can be easily applied to PEG tethered surfaces in relation to PEG solubility. To maximize hydration, water molecules have electron donor (oxygen) and electron acceptor (hydrogen) regions, [92]. The model predicts similar hydration. PEG is a soluble polymer with strong interactions with water molecules. As a result of this strong interaction, the basis of the chemical view of fouling resistance can be explained by the enthalpic penalty of stripping water molecules from the PEG, which cannot be easily overcome by proteins. Numerous investigations have been conducted in search of mechanisms by which surface bound PEG can restrict adsorption. There is agreement that PEG surface coatings, with sufficient chain length and packing density, can impart a broad non-biofouling characteristic to a biomaterial surface.

In this work, PEG was used as one of the surface coatings for the SiC-based Ti/Au antenna surface to resist protein adsorption, as well as biofouling.

\subsection{In vivo Study}

\subsubsection{Materials and Methods}

The Ti/Au antenna fabricated on a SI 4H-SiC substrate worked as a glucose sensor. In chapter 3, an in vitro glucose sensing mechanism was demonstrated using blood and fat mimicking liquid. Even though, all-SiC antenna approaches including - poly $3 \mathrm{C}-\mathrm{SiC}$ antenna electrode on semi-insulating (SI) 4H-SiC substrate did not show reasonable antenna performance (discussed in Chapter 2), we included this antenna for in vivo implantation to evaluate tissue histological analysis of this material. We have seen in the previous sections that bulk and surface material properties are very important for 
biomaterial applications in different implantable devices. Bulk properties, including mechanical, thermal, electrical, and the biocompatibility of $4 \mathrm{H}-\mathrm{SiC}$ as a substrate and $\mathrm{Ti} / \mathrm{Au}$ as an antenna electrode have been found to be suitable for this antenna sensor. For the in vivo study, we have selected 5 different antennas for implantation, Silicon and single crystal 3C-SiC and looked at surface biocompatibility through surface modifications and coatings. The implantable antennas are shown in Figure 30:

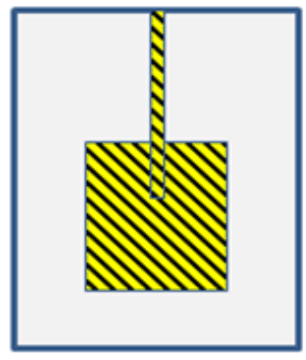

(a)

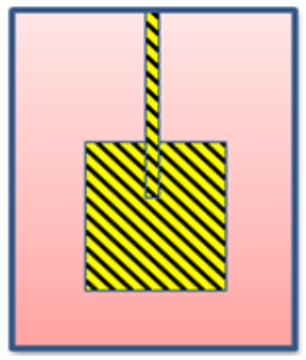

(b)

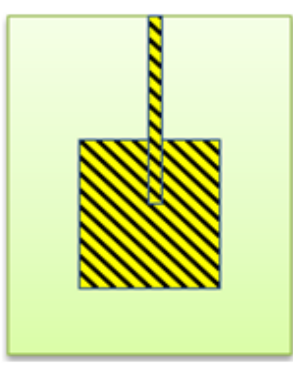

(c)

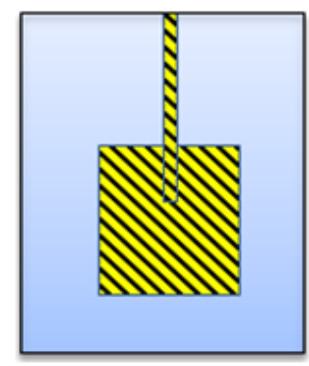

(d)

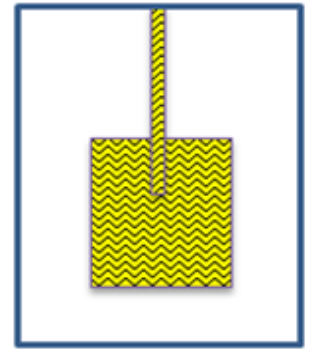

(e)

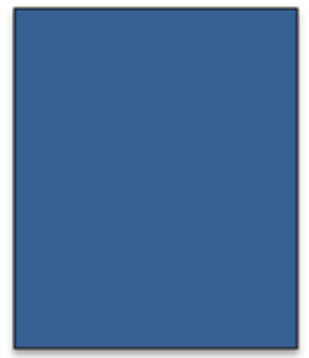

(f)

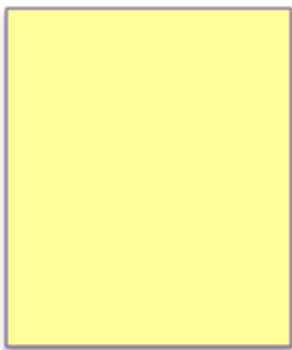

(g)

Figure 30: Seven different antenna surfaces and samples (a) Ti/Au antenna on SI 4H-SiC as the base. (b) SiO2 coated $\mathrm{Ti} / \mathrm{Au}$ antenna on $\mathrm{SI} 4 \mathrm{H}-\mathrm{SiC}$, (c) PEG coated Ti/Au antenna on $\mathrm{SI} 4 \mathrm{H}-\mathrm{SiC}$, (d) amorphous $\mathrm{SiC}(\mathrm{a}-\mathrm{SiC})$ coated $\mathrm{Ti} / \mathrm{Au}$ antenna on $\mathrm{SI} 4 \mathrm{H}-\mathrm{SiC}$, (e) all $\mathrm{SiC}$ antenna - poly 3C-SiC on SI 4H-SiC, (f) Silicon ( $\mathrm{Si}$ ), (g) 3C-SiC and a-SiC coat on the back.

All implant reactivity to cells/tissue was evaluated using tissue histology analysis. Biocompatibility and non-biocompatibility were verified using different inflammatory response analysis. For statistical analysis, a total of 28 different samples were implanted in a farm pig model, as shown in Table 4. 
Table 4: Details of in vivo experimental implants in pig model

\begin{tabular}{|c|c|c|c|c|c|c|}
\hline $\begin{array}{c}\text { Materials/De } \\
\text { vices }\end{array}$ & $\begin{array}{l}\text { Exper } \\
\text { iments }\end{array}$ & $\begin{array}{c}\text { Pig } 1 \\
\text { (Sample } \\
\text { No.) }\end{array}$ & $\begin{array}{c}\text { Pig } 2 \\
\text { (Sample } \\
\text { No.) }\end{array}$ & $\begin{array}{c}\text { Pig } 3 \\
\text { (Sampl } \\
\text { e No.) }\end{array}$ & $\begin{array}{c}\text { Pig } 4 \\
\text { (Sample } \\
\text { No.) }\end{array}$ & $\begin{array}{c}\text { Total pig(s) } \\
=4\end{array}$ \\
\hline $\begin{array}{l}\text { SiC based } \\
\text { Ti/Au antenna }\end{array}$ & \multirow{4}{*}{$\begin{array}{l}\text { Histol } \\
\text { ogical } \\
\text { Tissue } \\
\text { exami } \\
\text { nation }\end{array}$} & 1 & 1 & 1 & 1 & $\begin{array}{c}\text { Implanted } \\
\text { Device } 1=4\end{array}$ \\
\hline $\begin{array}{l}\text { PEG coated } \\
\text { Ti/Au antenna }\end{array}$ & & 1 & 1 & 1 & 1 & $\begin{array}{c}\text { Implanted } \\
\text { Device } 2=4\end{array}$ \\
\hline $\begin{array}{l}\mathrm{SiO}_{2} \text { coated } \\
\mathrm{Ti} / \mathrm{Au} \text { antenna }\end{array}$ & & 1 & 1 & 1 & 1 & $\begin{array}{c}\text { Implanted } \\
\text { Device } 3=4\end{array}$ \\
\hline $\begin{array}{l}\text { A- SiC coated } \\
\text { Ti/Au antenna }\end{array}$ & & 1 & 1 & 1 & 1 & $\begin{array}{c}\text { Implanted } \\
\text { Device } 4=4\end{array}$ \\
\hline $\begin{array}{l}\text { All SiC } \\
\text { antenna }\end{array}$ & \multirow{2}{*}{$\begin{array}{l}\text { AFM } \\
\text { analysi } \\
\text { s }\end{array}$} & 1 & 1 & 1 & 1 & $\begin{array}{c}\text { Implanted } \\
\text { Device } 5=4\end{array}$ \\
\hline Silicon & & 1 & 1 & 1 & 1 & $\begin{array}{c}\text { Implanted } \\
\text { Device } 6=4\end{array}$ \\
\hline $\begin{array}{l}\text { 3C-SiC; a-SiC } \\
\text { coated sample }\end{array}$ & $\begin{array}{l}\text { SEM } \\
\text { analysi } \\
\text { s }\end{array}$ & 1 & 1 & 1 & 1 & $\begin{array}{c}\text { Implanted } \\
\text { Device } 7=4\end{array}$ \\
\hline \multicolumn{2}{|l|}{ Total } & $\begin{array}{c}\text { 7devices } \\
\text { /pig } 1\end{array}$ & $\begin{array}{c}\text { 7devices } \\
\text { /pig } 2\end{array}$ & $\begin{array}{l}\text { 7device } \\
\text { /pig } 3\end{array}$ & $\begin{array}{c}\text { 7devices } \\
\text { /pig } 4\end{array}$ & 28 \\
\hline \multicolumn{6}{|c|}{ Total implanted samples in $\mathbf{4}$ pigs } & 28 \\
\hline
\end{tabular}

\subsubsection{Sample Preparation on SiC-based Ti/Au Antenna}

To maintain animal numbers to a strict minimum, we have selected the materials which have already been proved earlier for in vitro bio-compatibility. It has been shown by our group previously that $\mathrm{SiC}$ has excellent compatibility with skin and connective 
tissue cells externally $[30,32]$. It has also been shown that the bio-compatibility of covalently bonded Poly ethylene glycol (PEG) can remarkably reduce the non-specific adsorption of protein [18]. Titanium (Ti), silicon-dioxide $\left(\mathrm{SiO}_{2}\right)$ and inert material gold $\mathrm{Au})$ are frequently used for different implantations. For post-implant histological tissue analysis such as inflammation or fibrosis capsule development, different surface coatings/surface modifications were used on $\mathrm{SiC}$ based antenna sensors.

a) The detail fabrication procedure of $\mathrm{Ti} / \mathrm{Au}$ antenna on $\mathrm{SI} 4 \mathrm{H}-\mathrm{SiC}$ as the base sensor was explained in Section 2.2. Briefly, the antenna was prepared by depositing Ti (500 $\AA$ ) and $\mathrm{Au}(4500 \AA ̊)$ using E-beam evaporator after cleaning the SI 4H-SiC substrate. Finally the antenna was patterned using standard photolithography. Here, all the antenna materials titanium, gold and $4 \mathrm{H}-\mathrm{SiC}$ are biocompatible. The schematic side view of the antenna is shown in Figure 31. The measured antenna response has already been shown in Figure 8 .

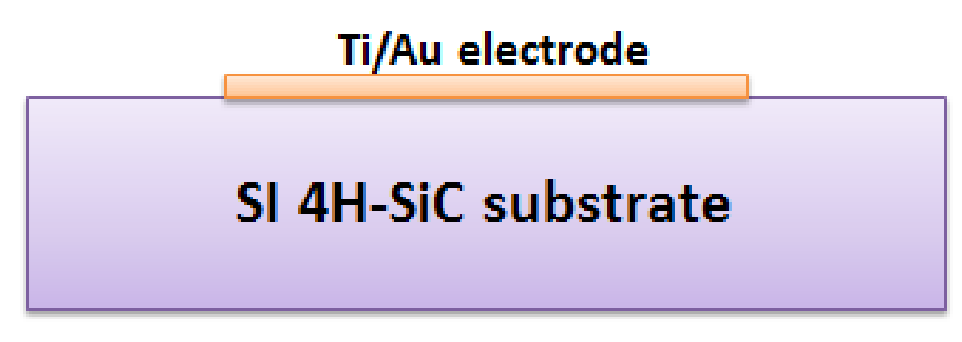

Figure 31: Cross-section view of the $\mathrm{SiC}$ based $\mathrm{Ti} / \mathrm{Au}$ antenna

b) $\mathrm{SiO}_{2}$ coated $\mathrm{Ti} / \mathrm{Au}$ antenna on $\mathrm{SI} 4 \mathrm{H}-\mathrm{SiC}$ was prepared by depositing $\mathrm{SiO}_{2}$ on $\mathrm{Ti} / \mathrm{Au}$ antenna using the plasma enhanced chemical vapor deposition (PECVD) technique, the schematic side view is shown in Figure 32. One of the reasons for choosing this $\mathrm{SiO}_{2}$ coated sample is to reduce direct adhesion problems of other biocompatible material coatings such as $a-\mathrm{SiC}$ and poly ethylene glycol (PEG) on the Ti/Au antenna 
surface. The antenna performance was measured after $\mathrm{SiO}_{2}$ deposition which is shown in Figure 34 (b). In addition to this, the poly 3C-SiC antenna approach used a $\mathrm{SiO}_{2}$ coating underneath the seed poly $\mathrm{Si}$ layer [frewin et al]. Therefore, we have decided to implant a $\mathrm{SiO}_{2}$ coated sample to directly observe the tissue histological response after 30 days of implantation.

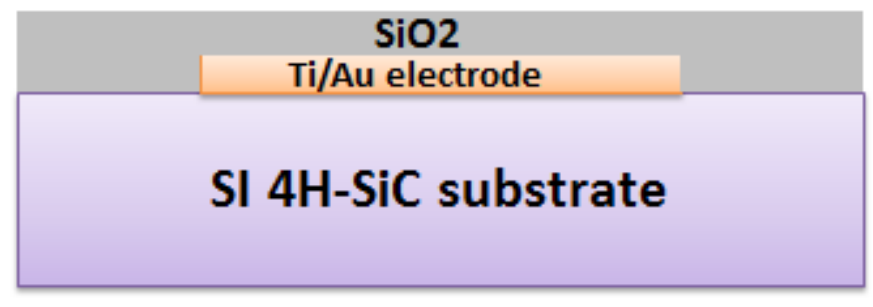

Figure 32: Cross-section view of the $\mathrm{SiO}_{2}$ coated $\mathrm{SiC}$ based $\mathrm{Ti} / \mathrm{Au}$ antenna.

c) As stated earlier, oligo- or poly(ethyleneglycol) (PEG) is the most "inert" chemical group to protein adsorption. The hydrophilic nature of PEG attributes to this inertness or non-biofouling property of PEG. The formation of a partially structured $\mathrm{H}_{2}$ layer is the result of one of the properties of PEG which is extensively hydrogen-bonded to water molecules. The disruption of a structured water layer is needed for the adsorption of a protein molecule which is enthalpically inhibited. Also the protein adsorption leads to the compression of the PEG layer towards the solid surface which is entropically unfavorable. As a result, for the immobilization of protein molecules, as inert surfaces PEG and other oligo- or poly-ethers have been successfully used. For examples Whitesides' group and others used PEG films anchored to glass or other oxide terminated surfaces by silane coupling chemistry and showed that this works as a protein inhibitor [16]. Silane chemistry based PEG suffers from instability and low PEG density due to hydrolysis. To overcome these limitations, A proprietary process 
has been successfully developed to synthesize a high density brush of polyethyleneglycol (PEG). As compared to PEG from conventional silane coupling chemistry, the density of grafted PEG groups is $1-2$ orders of magnitude higher. And this high PEG density increases the exceptional repulsiveness of the surface towards protein adsorption, shown in Figure 33.

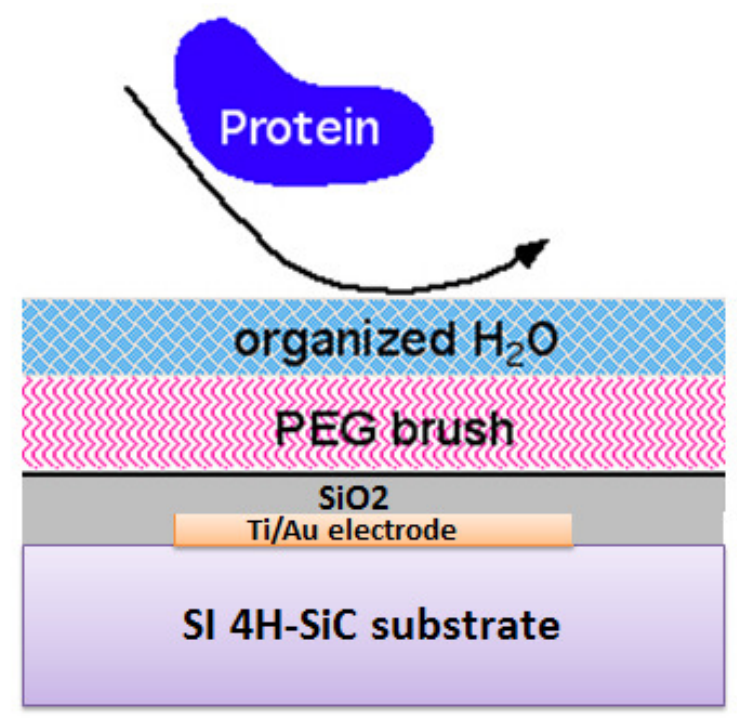

Figure 33: Cross-section of the PEG coated SiC based Ti/Au antenna. The repulsion of proteins is shown to illustrate the process.

d) Biocompatible amorphous $\mathrm{SiC}(a-\mathrm{SiC})$ thin film coating of $\mathrm{Ti} / \mathrm{Au}$ antenna is another approach to make the antenna surface biocompatible. The confirmation of biocompatibility of $a$-SiC has already been discussed in section 1.2 . The $120 \mathrm{~nm}$ thick $a$-SiC coating was deposited on $3 \mu \mathrm{m} \mathrm{SiO}_{2}$ coated $\mathrm{Ti} / \mathrm{Au}$ antenna surface by using plasma enhanced chemical vapor deposition (PECVD). 


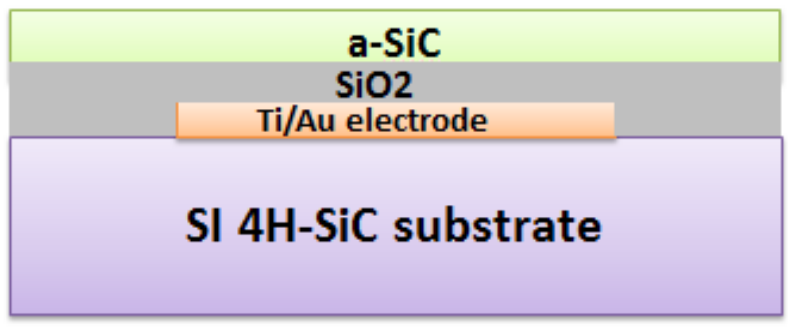

(a)

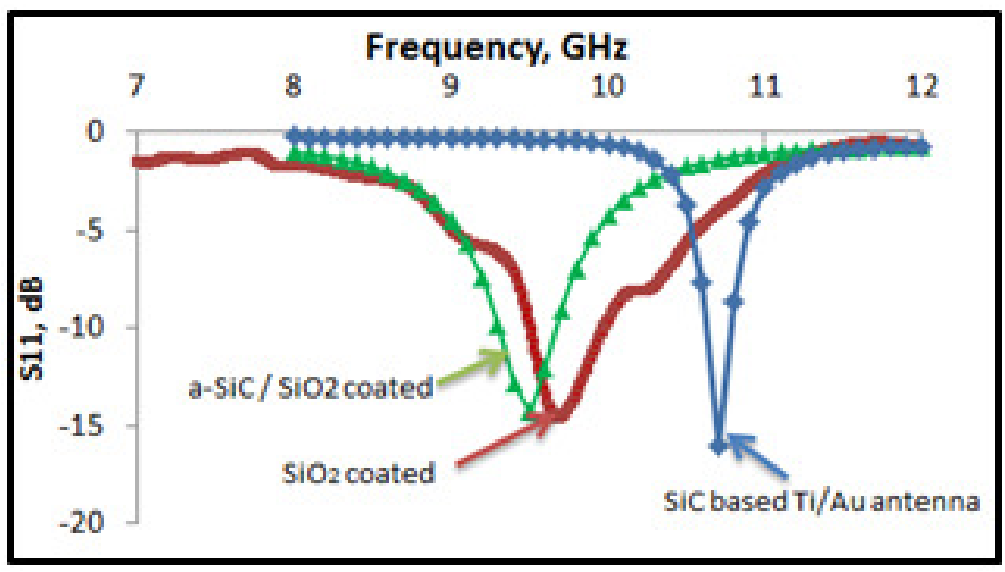

Figure 34: (a) Cross-section of the a-SiC coated $\mathrm{SiC}$ based $\mathrm{Ti} / \mathrm{Au}$ antenna. (b) Experimental Return loss of $\mathrm{SiO}_{2}$ coated and a-SiC coated $\mathrm{Ti} / \mathrm{Au}$ antennas showing a shift to lower resonant frequency as expected due to the higher dielectric constant of these coatings compared to air

The coating was deposited all over the antenna surface (top layer - $\mathrm{Au}$ and $4 \mathrm{H}-\mathrm{SiC}$ ) to make sure the a-SiC coating was uniform, as is shown in Figure 34 (a). After $a$-SiC coating, the antenna performance was measured using the VNA, (as explained in 2.2.1), and the results shown in Figure 34 (b).

e) Finally the all $\mathrm{SiC}$ antenna, poly $3 \mathrm{C}-\mathrm{SiC}$ on $\mathrm{SI} 4 \mathrm{H}-\mathrm{SiC}$, which was described in section 3.2.was implanted. Briefly the heavily $\mathrm{N}_{2}$ doped poly $3 \mathrm{C}-\mathrm{SiC}$ thin film was grown on a poly-Si/SiO $/ \mathrm{Si}_{2} / \mathrm{Si}$ coated substrate using the USF SiC Group $\mathrm{SiC}$ CVD reactor and then fabricated using standard photolithography. The side view schematic is shown in Figure 35. The antenna response has shown in Figure 16. 


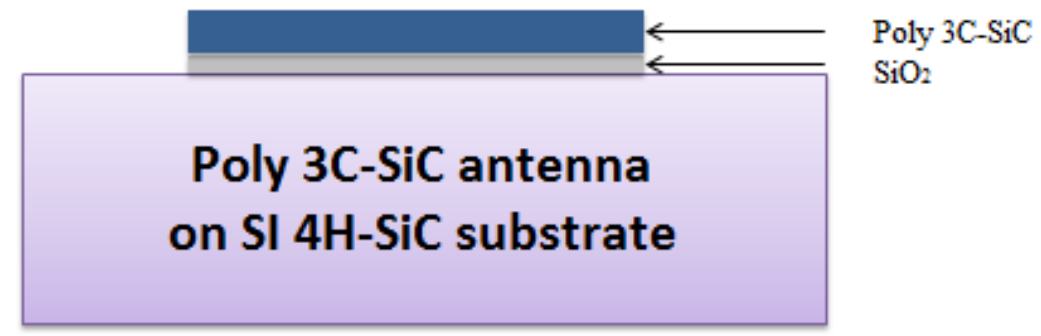

Figure 35: Cross-section of the Poly 3C-SiC antenna showing all of the materials used to realize the antenna.

After preparing the samples, the surface morphology was characterized using AFM [XE-100 Advanced Scanning Microscope (AFM) from Park Systems®]. Figure 36 displays AFM micrographs of the surfaces of the implants before subcutaneous implantation in the pig. AFM was performed in tapping mode with a scan size $10 \times 10$ $\mu \mathrm{m}$, using silicon nitride probes.

The surface root mean square roughness, $\mathrm{r}_{\mathrm{q}}$ within 10 x $10 \mu \mathrm{m}$ was $2.36 \mathrm{~nm}$ RMS for e-beam evaporated Ti/Au antenna which is shown in Figure 36 (a) The PECVD $\mathrm{SiO}_{2}$ layer possesses a root mean square roughness of $13 \mathrm{~nm}$ RMS [Figure 36 (b)]. On top of $\mathrm{SiO}_{2}$ layer, a-SiC was deposited and the AFM surface roughness of $28.84 \mathrm{~nm}$ RMS was measured, shown in Figure 36 (c). Because of the large grain size the root mean square roughness of poly 3C-SiC was $29.26 \mathrm{~nm}$ RMS [Figure 36 (d)]. Due to the $20 \%$ lattice mismatch between 3C-SiC and substrate - Si, the 'as grown' 3C-SiC has rough, mosaic surface of roughness $3.96 \mathrm{~nm}$ RMS. Chemical-mechanical polished (CMP) Silicon is atomically flat, the AFM roughness was $0.5 \mathrm{~nm}$ RMS. 


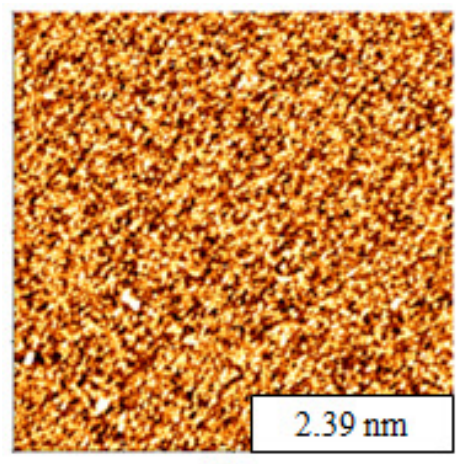

(a)

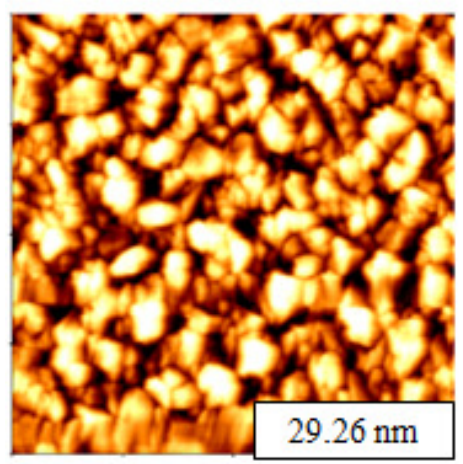

(d)

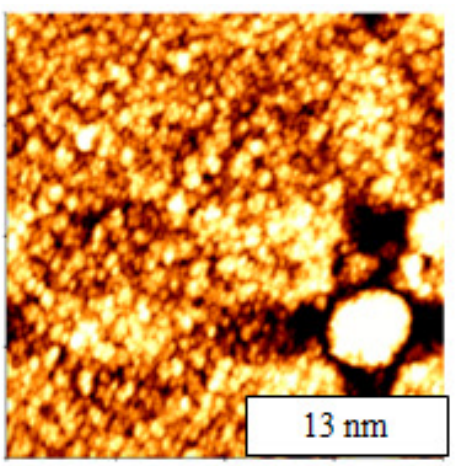

(b)

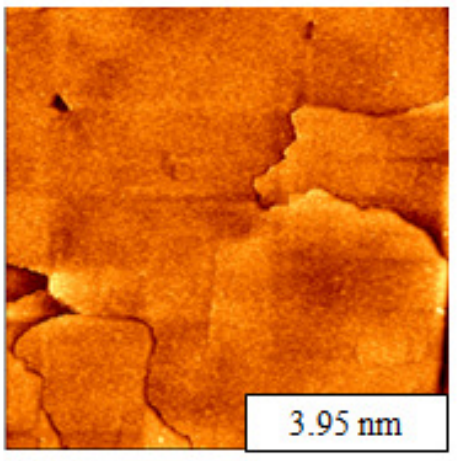

(e)

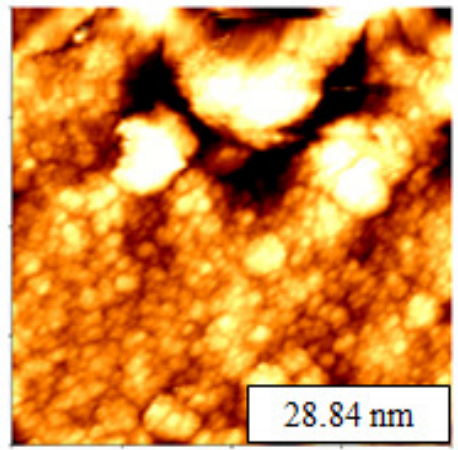

(c)

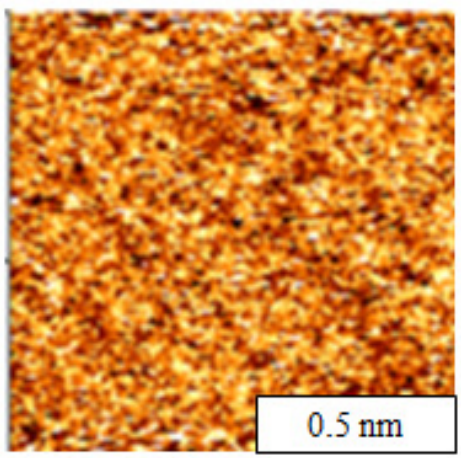

(f)

Figure 36: Pre-implant AFM micrographs. (a) $\mathrm{SiC}$ based $\mathrm{Ti} / \mathrm{Au}$ antenna; (b) $\mathrm{SiO}_{2}$ coated $\mathrm{Ti} / \mathrm{Au}$ antenna; (c) a-SiC deposited on $\mathrm{SiO}_{2}$ coated Ti/Au antenna; (d) Poly 3C-SiC (all$\mathrm{SiC}$ ) antenna; (e) Single crystal 3C-SiC - negative control; (f) Silicon - positive control Surface roughness root mean square (RMS) values are located in the lower right corner of each micrograph.

\subsection{In vivo Implantation}

\subsubsection{Implantation Procedure}

Prior to animal use for this research, a protocol was approved by the University of South Florida, Institutional Animal Care \& Use Committee (IACUC) which is included in Appendix B. After cleaning and disinfecting the implantable devices following standard practice and procedures, the devices were ready for subcutaneous implantation. We have selected four adult healthy mature male pigs of $20-35 \mathrm{Kg}$ weight. The pigs were housed for 7 days before the surgery according to standard regulations. We have selected pigs for this research for some important reasons. First, the pig model has long 
been a good tool to help medical researchers to mimic human body size and physiology in order to answer clinical questions. Furthermore our sensor size is not suitable for small animal like the mouse/rat, so the size of the sensor is suitable for implantation in a pig. All animals have been assigned to category B because they were anesthetized during the procedure.

After cleaning and disinfecting the implantable devices, they were ready for subcutaneous implantation. Prior to the induction of anesthesia, the pigs fasted for 12 hours. Carprofen was administered subcutaneously at the dose rate of $4.0 \mathrm{mg} / \mathrm{kg}$. Also prior to induction of general inhalational anesthesia, the animals received diazepam (at the initial dose rate of $4.0 \mathrm{mg} / \mathrm{kg}$ body weight $\mathrm{PO}$ ) and atropine sulfate (at the dose rate of $0.05 \mathrm{mg} / \mathrm{kg} \mathrm{IM}$ ). Ketamine was administered IM at the dose rate of $20 \mathrm{mg} / \mathrm{kg}$ body weight. This combination provided immobilization and sedation, permitting the staff to place a facemask over the snout with the animal comfortable for final induction and intubation under isoflurane (2-4\%) inhalational gas. Upon induction of general anesthesia, an endotracheal tube was placed, and the animal was connected to a positive displacement ventilator. An IV catheter was placed in the marginal ear vein of each ear to provide IV access and permit administration of fluids and IV medications as needed. Isoflurane (2-4\%) anesthesia was maintained and adjusted as needed to ensure full animal anesthesia. EKG leads, pulse oximetry sensor, and rectal temperature probes were mounted on the animal for vital sign monitoring. The depth of the anesthesia was ensured by using sensitive interdigital pinch and the palpebral/eyeblink reflex. Also the lack of reflex movement of the animal in response to noxious stimuli during the incisions indicated the level of anesthesia. The surgical sites were the mid posterior aspect- 
shoulder ( 2 devices), mid thoracic pave vertebrae ( 2 devices), mid lumber pave vertebrae (2 devices), mid sacral (1 device) and they were scrubbed at least twice with a germicidal scrub, being careful to scrub from the center of the site toward the periphery. The sites were then rinsed / wiped with a 70\% alcohol and then painted with dilute, tamed iodine solution. Then the area was draped with sterile drapes.
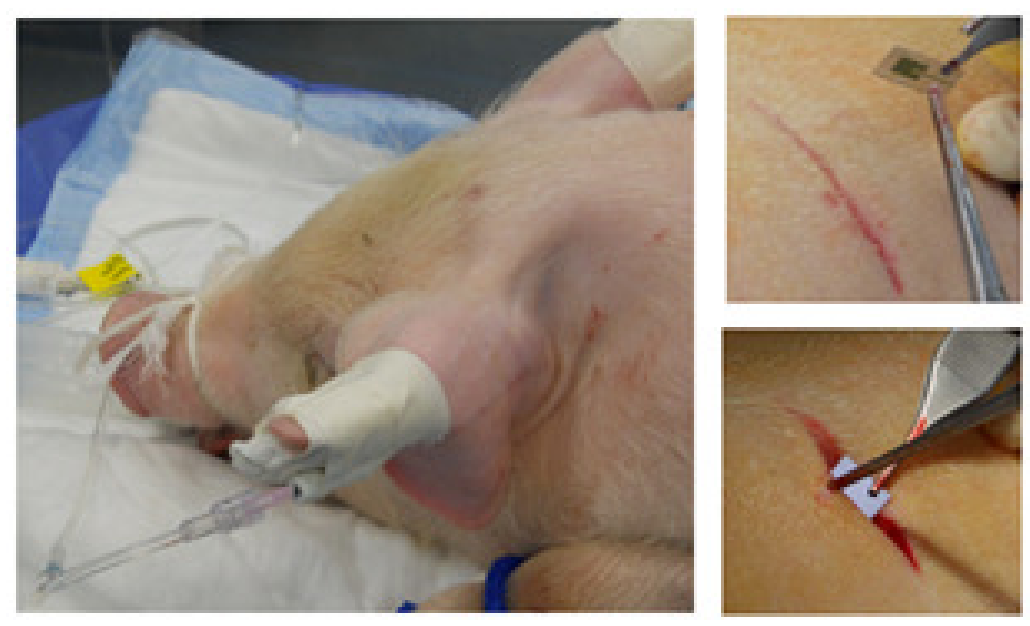

(a)

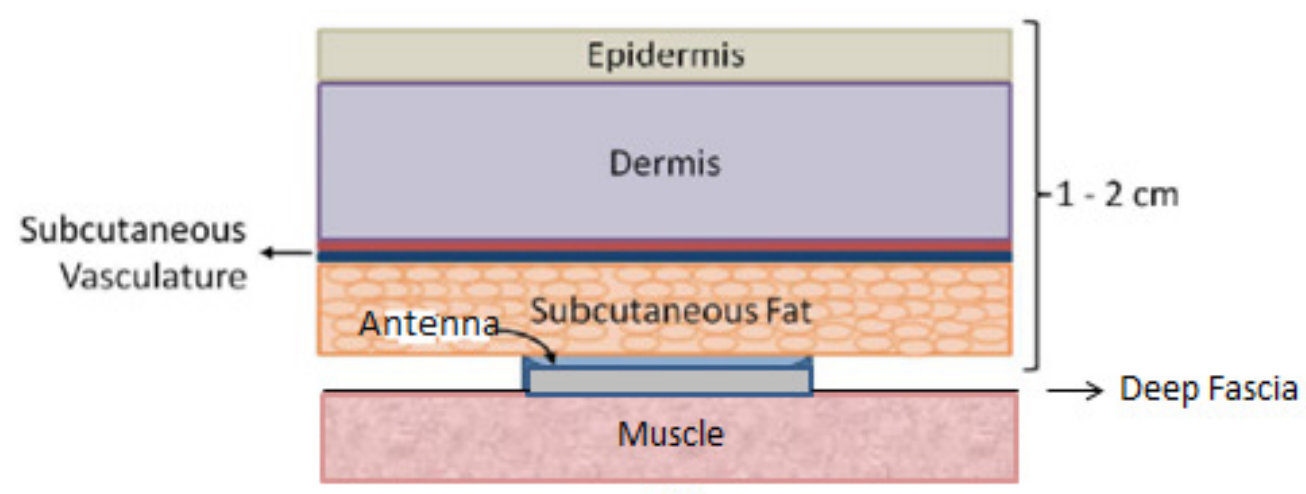

(b)

Figure 37: (a) Photograph of the subcutaneous implantation of the antenna sensor in pig. Each implant was placed into a pocket created between subcutaneous fat and underline fascia/muscle via blunt dissection exposing the layers of the skin as shown in the lowerright hand photo and (b) cross-section illustration 
After preparation of the surgical site $1.5 \times 1$ centimeter $^{2}$ pockets were created beneath the skin (dermis) surface. The implants were then placed into the pocket very carefully using fine tweezers which is shown in Figure 37.

Post-operatively, the animals were monitored continuously and assessed using systemic temperature, pulse rate, respiratory rate, mucous membrane color, and capillary refill time. Animals were recovered in a clean separate place with ancillary heat control. This continued until the animals were eternally recumbent and capable of voluntary movement before being transferred to the housing area. Following implantation, and recovery from anesthesia, the animals were housed in a single housing area for 30 days, with routine nourishment, monitoring and evaluated every day. A log of observation was kept for proper monitoring. This single housing was designed to minimize the risk of surgical site trauma resulting from animal-animal interactions. All assessments were recorded on forms provided by Comparative Medicine at University of South Florida.

\subsubsection{Implants Removal and Tissue Collections}

After 30 days, post implant, the animals were again placed under general anesthesia. The surgical incisions were re-opened, and the previously placed implants were removed for analysis. The pigs were then euthanatized using an IV bolus of 1 to $2 \mathrm{mmol} / \mathrm{kg}$ (at least $30 \mathrm{ml})$ USP grade of saturated potassium chloride $(\mathrm{KCl})$ overdose while still under general anesthesia. Death was confirmed by verification of cessation of cardiac and respiratory activity. Immediately after that the surrounding tissue were removed for analysis. A total of 29 tissue blocks, 28 implants from four pigs and 1 fresh tissue block to compare were extracted. The specimens were fixed in $4 \%$ paraformaldehyde solution 
after retrieval. During the removal of the implants it was observed that, in general, most of the implants were easily moved across the skin which is indication of no encapsulation or fluid around the wounds. The positive control $\mathrm{Si}$ and $\mathrm{SiO}_{2}$ coated samples had fibrosis capsules around the implants.

After removal, the implants were cleaned using the piranha cleaning method. Any cellular/tissue remnants, extracellular proteins, etc. were completely removed when placed in $75^{\circ} \mathrm{C}$ piranha solution $\left(2: 1 \mathrm{H}_{2} \mathrm{SO}_{4}: \mathrm{H}_{2} \mathrm{O}_{2}\right)$ for 10 minutes. The implants were immediately rinsed 3 times in DI water.

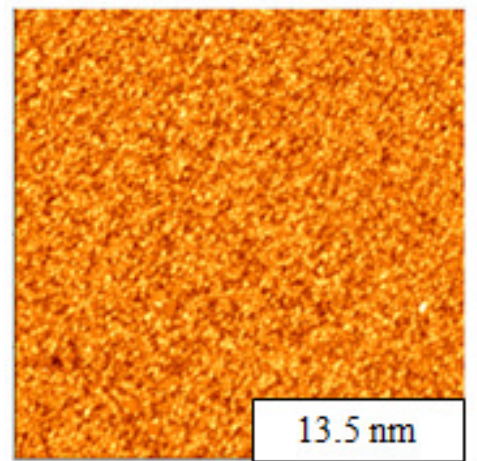

(a)

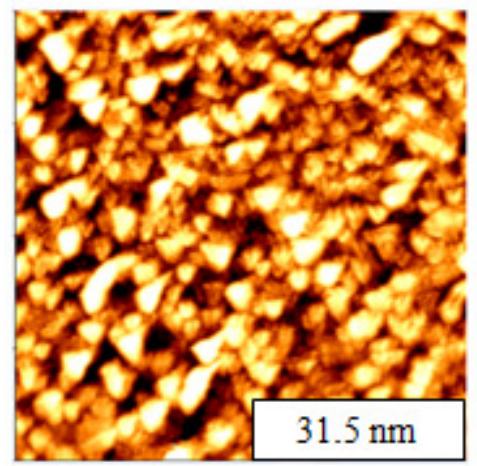

(d)

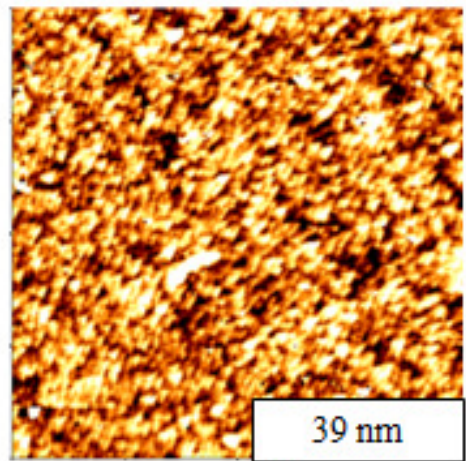

(b)

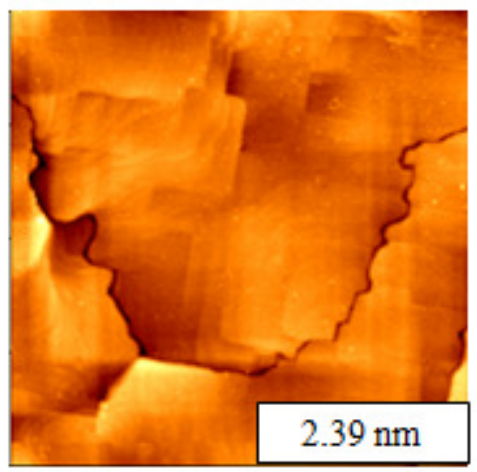

(e)

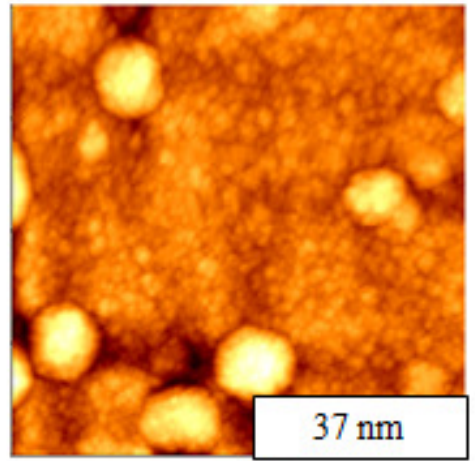

(c)

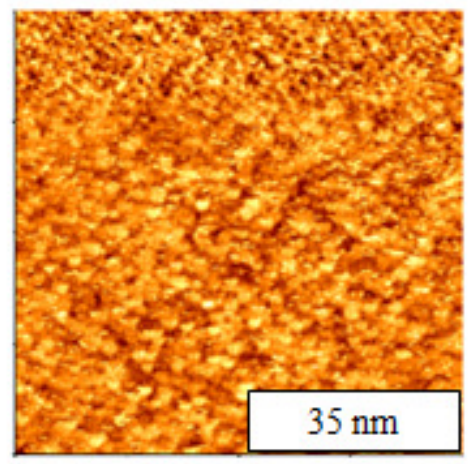

(f)

Figure 38: Post implant AFM micrographs. (a) $\mathrm{SiC}$ based $\mathrm{Ti} / \mathrm{Au}$ antenna; (b) $\mathrm{SiO}_{2}$ coated $\mathrm{Ti} / \mathrm{Au}$ antenna; (c) $a$-SiC deposited on $\mathrm{SiO}_{2}$ coated Ti/Au antenna; (d) Poly 3C-SiC (allSiC) antenna; (e) Single crystal 3C-SiC - negative control; (f) Silicon - positive control Surface roughness root mean square (RMS) values are located in the lower right corner of each micrograph. Scan size is $10 \times 10 \mu \mathrm{m}$. 
The samples were then dried using pressurized $\mathrm{N}_{2}$. Post implant surface morphology was evaluated using AFM which is shown in Figure 38. AFM was performed in tapping mode with a scan size $10 \times 10 \mu \mathrm{m}$, using silicon nitride probes. Surface roughness root mean square (RMS) values are located in the lower right corner of each micrograph. As $\mathrm{SiC}$ material is chemically inert, the surface roughness of $3 \mathrm{C}-\mathrm{SiC}, a-\mathrm{SiC}$ and poly $3 \mathrm{C}-\mathrm{SiC}$ had similar root mean square values before and after implantation. The roughness of $\mathrm{Au}$, $\mathrm{SiO}_{2}$ and $\mathrm{Si}$ were increased from before implantation to after implantation by $11 \mathrm{~nm}$ RMS, 26 nm RMS and 34.5 nm RMS, respectively.

\subsection{Histological Tissue Examination}

The study of the microscopic anatomy of animal cells and tissues is known as histology. The light microscope procedure was used in this research for tissue examination whereby a white light beam was transmitted through the tissue and the image captured using a digital camera. Normally the tissue is too thick for light to pass through after extraction from the animal. In histological studies, it is very important to obtain thin, translucent tissue sections. Therefore, tissue blocks are dehydrated in a series of ethanol (from $10 \%(\mathrm{v} / \mathrm{v})$ to $100 \%$ ) and embedded in paraffin. A microtome was then used to prepare thin $(5 \mu \mathrm{m})$ sections in a transversal direction to the axis of the implant after polymerization (details of this sectioning is included in Appendix C). For each tissue block, histological sections were made. The sections were stained with hematoxylin and eosin for conducting histological studies (details included in Appendix D). Hematoxylin stains the nucleus a dark purple-blue and eosin stains the other cells pink, especially cytoplasm. It was noticed that most of the implants retained their original 
shape and stayed in place during the implantation period. There was no sign of breakage or deformation of the implants (total 28 implants) other than one implant, an $a$-SiC coated $\mathrm{SiC}$ based $\mathrm{Ti} / \mathrm{Au}$ antenna, which unfortunately broke into two pieces.

As stated earlier 28 implants from four pigs were extracted with a total of 29 tissue blocks (one for each implant and a fresh tissue block to serve as a control) were extracted. The light micrograph of the fresh tissue block without any implant is shown in Figure 39 for reference. Most of the tissues have been compared with this healthy tissue response.

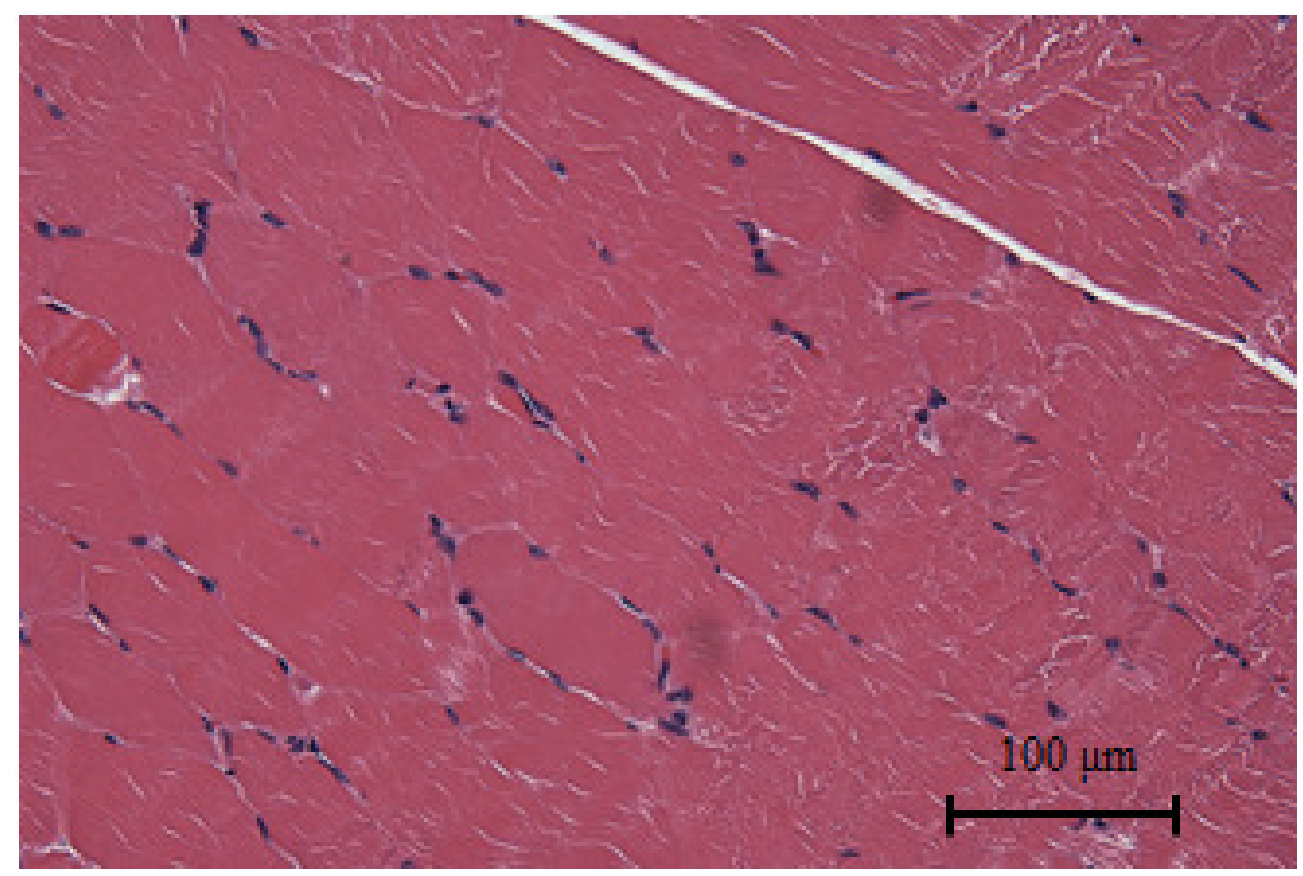

Figure 39: Histological tissue analysis of healthy tissue without any implant showing no signs of inflammation (original magnification x40). (Candance A. Miranda and Dr. K. Muffly helped in tissue preparation and data collection respectively in Dr. K. Muffly's laboratory, USF College of Medicine)

Histological examination of a subcutaneous implant $\mathrm{Si}$ (positive control) revealed that the adverse tissue response occurred to all four implants and this response was fairly uniform at 4 weeks. All 4 Si implants had a thin circumferential fibrous capsule around 


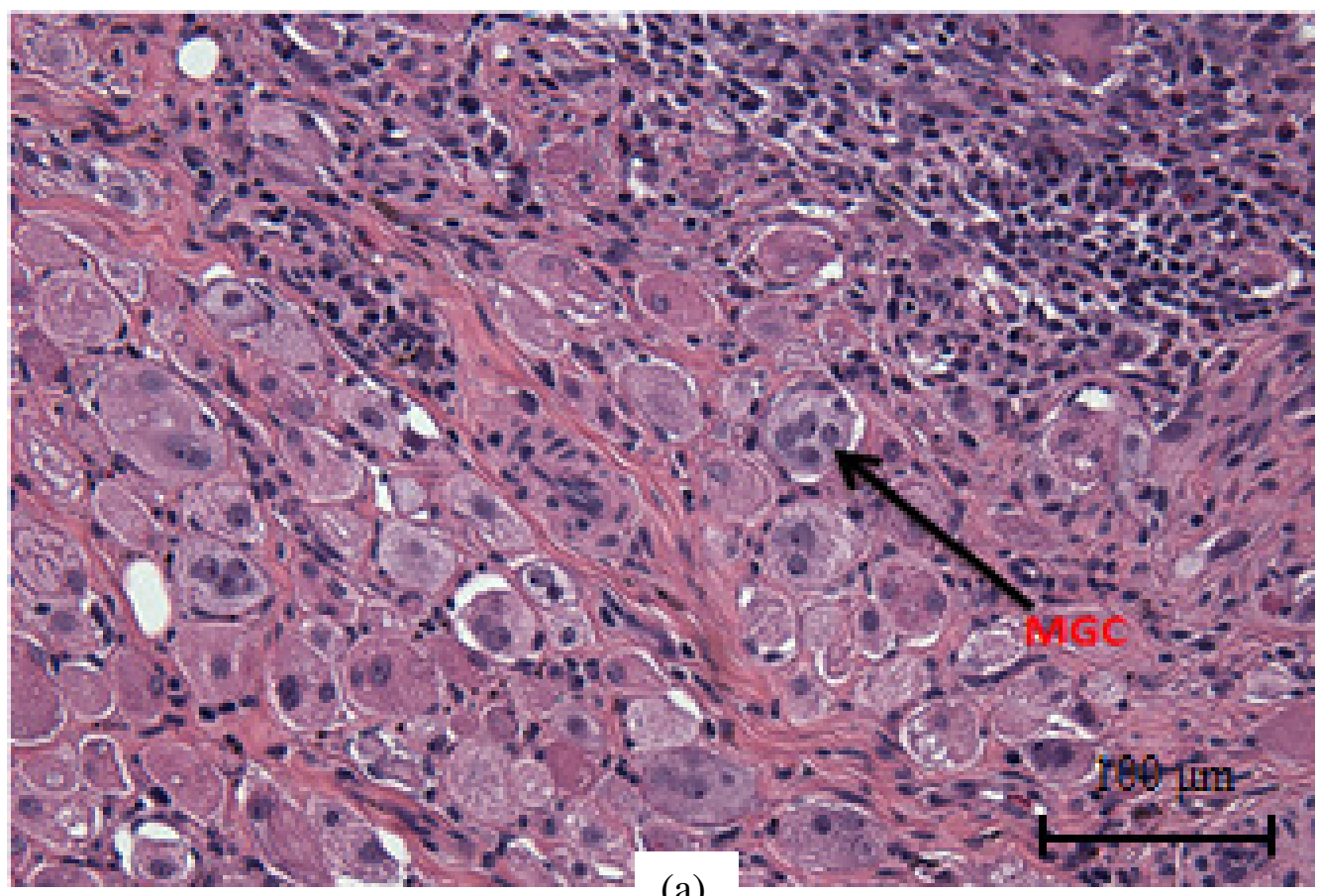

(a)

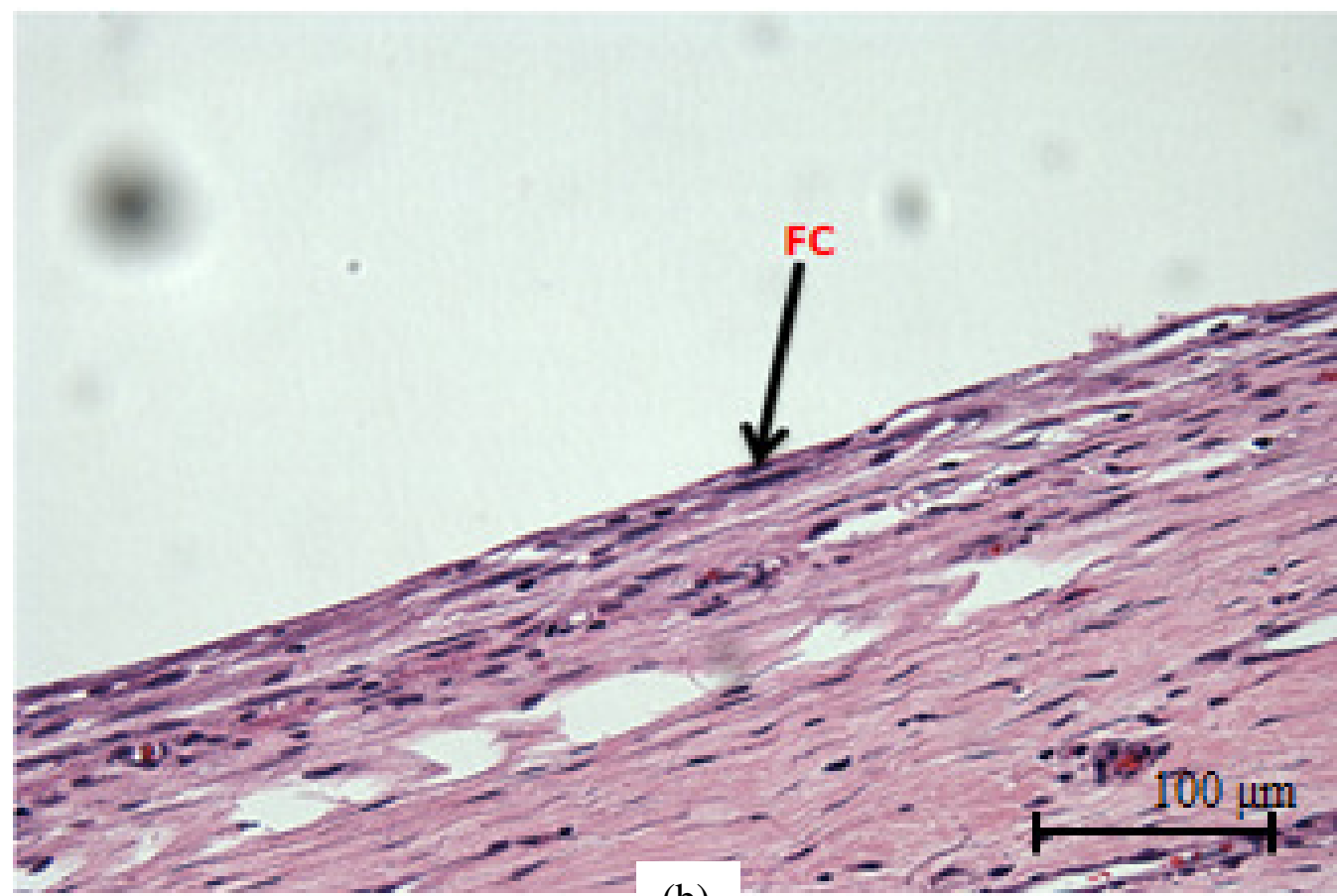

(b)

Figure 40: Histological tissue analysis of positive control $\mathrm{Si}$. (a) initial stage of inflammatory infiltration with macrophages, multinucleated giant cells (MGC); (b) there was almost no inflammatory cell infiltration in the tissue; thus, the tissue began to change to fibrous encapsulation (FC) (30 days-specimen, x40). (Candance A. Miranda and Dr. K. Muffly helped in tissue preparation and data collection respectively in Dr. K. Muffly's laboratory, USF College of Medicine). 
them which is shown in Figure 2 (b). These fibrous capsules mainly consisted of a few inflammatory cells and flattened fibroblasts. Furthermore, organized connective tissue and mature fibroblasts also surrounded the implants. On the other hand, some inflammatory infiltration signs, such as marcrophages and giant cells (shown in Figure 40 (a)) were observed in different regions of the tissue. Ultimately these can lead to the formation of fibrosis tissue and finally layers of fibrous capsule; however 4 weeks is insufficient time to see this 'long-term' acute response but clearly this is the expected outcome when these types of tissue modifications are observed.

The Silicon implants were surrounded by fibrous capsules; after removal of the implant the shape of the capsule was the same as the implant which is shown in Figure 41.

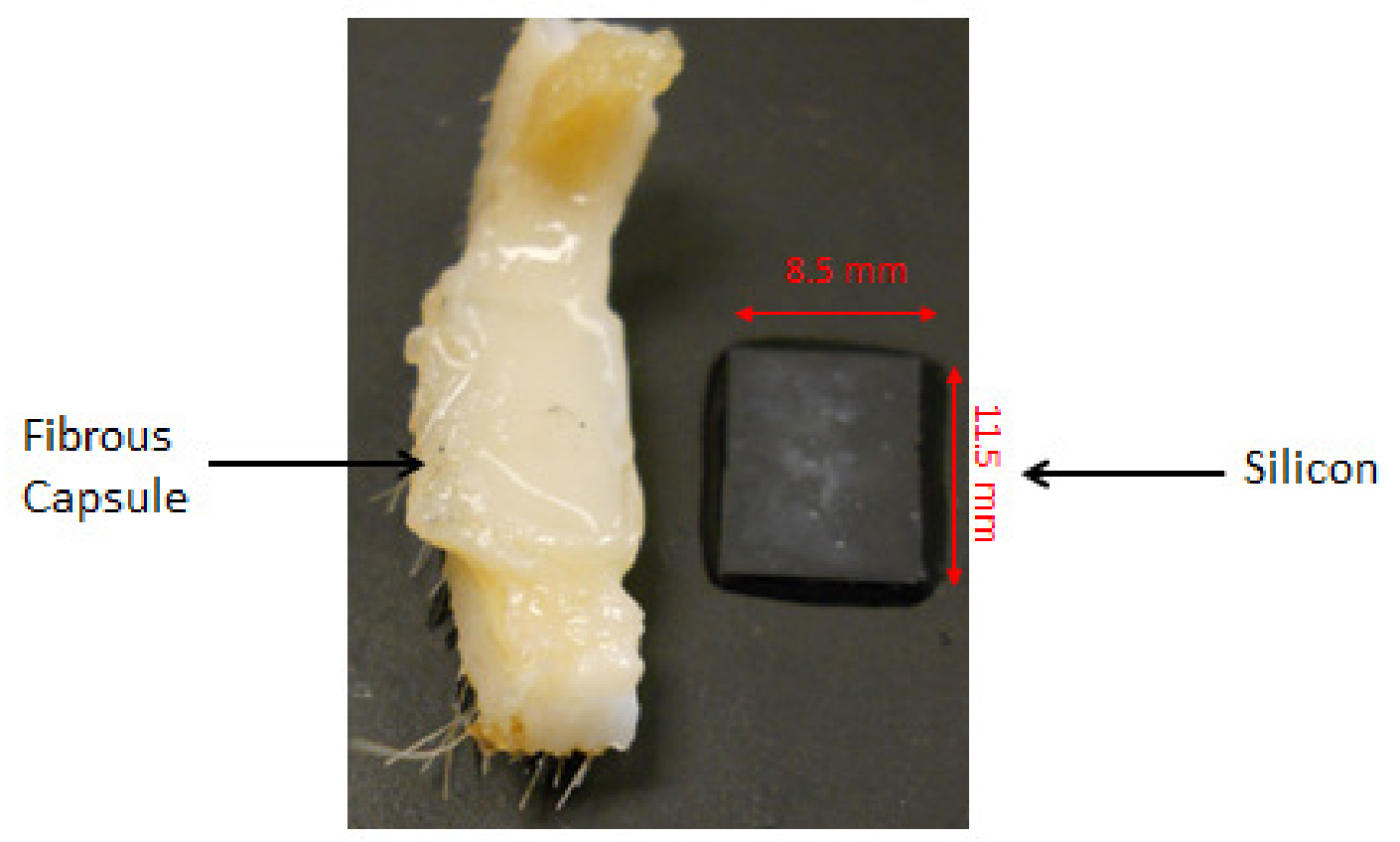

Figure 41: Photograph of the fibrous capsule after removal Si implant (30 days in vivo implantation specimen). 


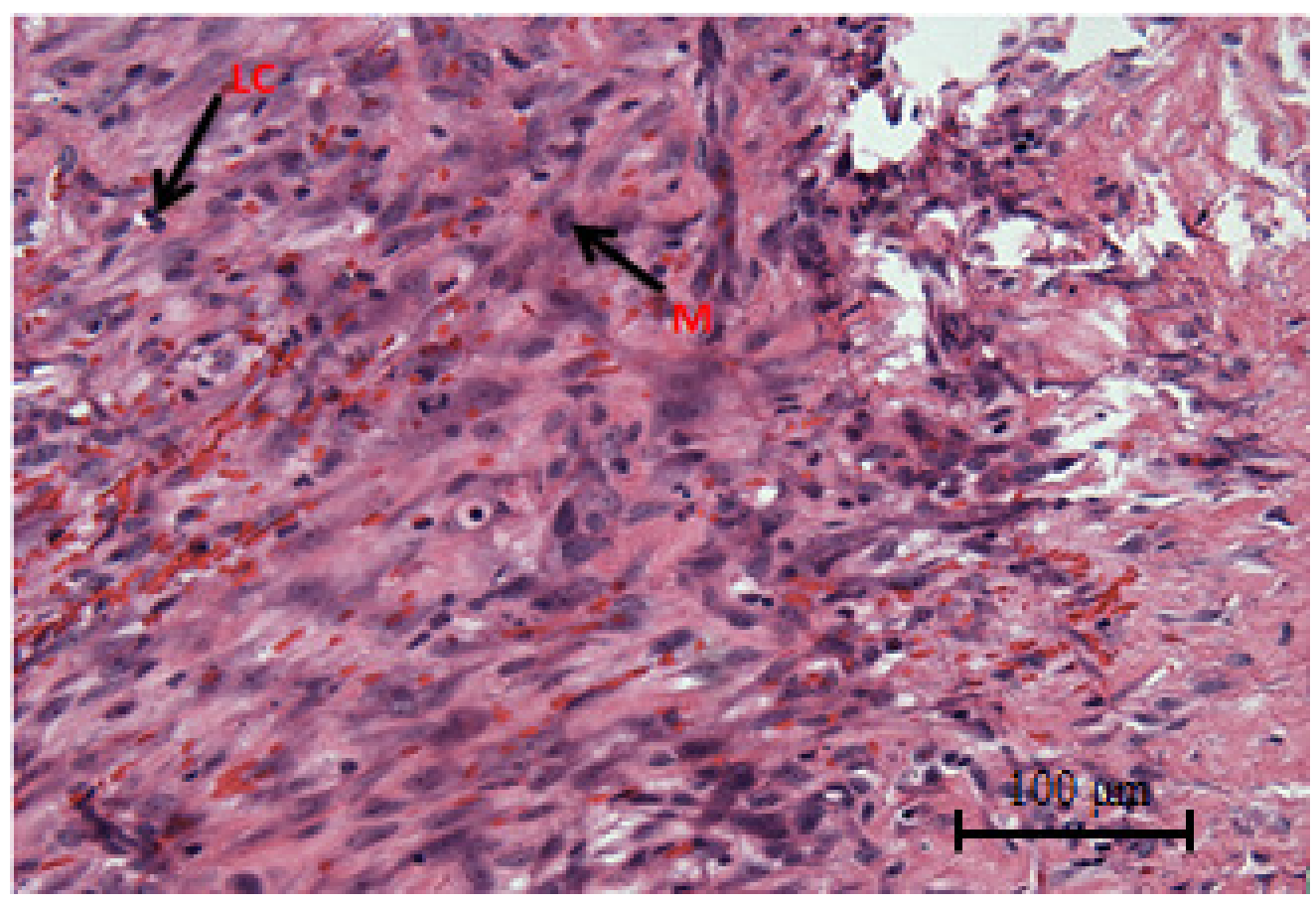

Figure 42: Histological tissue analysis of the bare Ti/Au antenna. Proliferation of lymphocytes (LC), macrophages (M), (30 days-specimens, x40). (Candance A. Miranda and Dr. K. Muffly helped in tissue preparation and data collection respectively in Dr. K. Muffly's laboratory, USF College of Medicine).

A SiC-based Ti/Au antenna (no additional coating) was one of the implants studied.

After 30 days of subcutaneous implantation, the $\mathrm{Ti} / \mathrm{Au}$ antenna demonstrated accumulation of inflammatory cells and macrophages at the implant-tissue interface (shown in Figure 42), which is a sign of acute inflammatory response. However there was no sign of fibrous capsule development around the embedded material after 4 weeks of implantation.

The tissue histology of the all $\mathrm{SiC}$-antenna, which consists of poly $3 \mathrm{C}-\mathrm{SiC}$ as the antenna electrode and $4 \mathrm{H}-\mathrm{SiC}(\mathrm{SI})$ as the susbstrate, had one of the most promising results. There was no sign of inflammatory response for all 4 implants. This tissue histological result (Figure 43), from the all-SiC antenna, is comparable to the fresh tissue shown in Figure 39. 


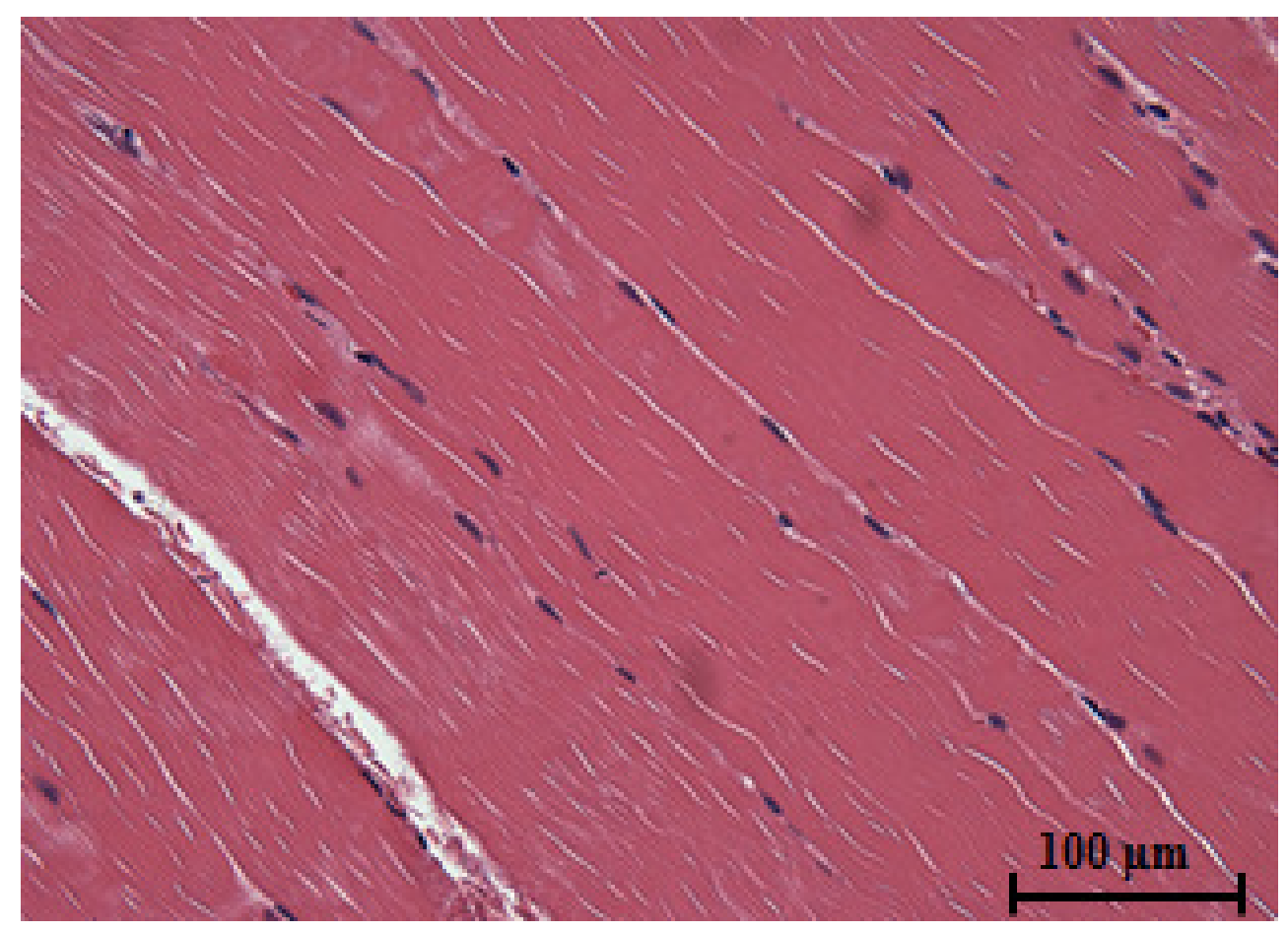

Figure 43: Histological tissue analysis of the all-SiC antenna. No sign of inflammation (30 days-specimen, x40). (Candance A. Miranda and Dr. K. Muffly helped in tissue preparation and data collection respectively in Dr. K. Muffly's laboratory, USF College of Medicine)

The histological tissue analysis of the $\mathrm{SiO}_{2}$ coated $\mathrm{SiC}$-based $\mathrm{Ti} / \mathrm{Au}$ antenna specimen had similar trends as observed for the Si implants. Multiple nucleated giant cells, macrophages, fibroblasts, lymphocytes and evenly distributed eosinophil cells were evident which had a strong inflammatory cell infiltration, as shown in Figure 44 (a). The tissue in contact with the implant began to change to fibrous tissue. In addition the surface of the implanted material $\left(\mathrm{SiO}_{2}\right.$ coated $\mathrm{Ti} / \mathrm{Au}$ antenna) had a thin fibrous tissue layer, which covered the scattered connective tissues, as shown in Figure 44 (b). 

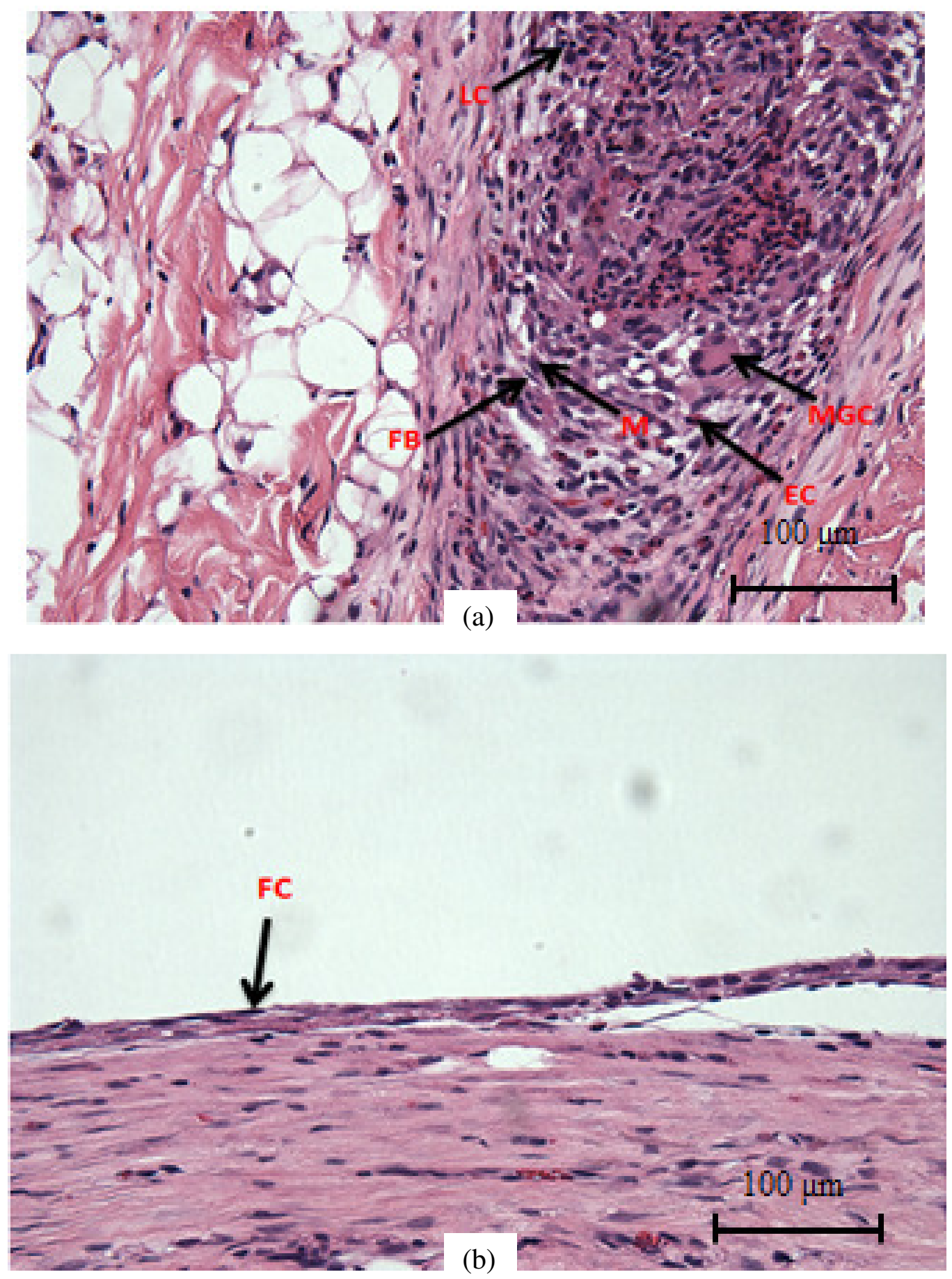

Figure 44: Histological tissue analysis of $\mathrm{SiO}_{2}$ coated $\mathrm{SiC}$-based $\mathrm{Ti} / \mathrm{Au}$ antenna. (a) macrophage (M), multinucleated giant cell (MGC), fibroblast (FB), lymphocyte (LC) with eosinophil cells (EC) are the sign of inflammatory response, (b) the tissue began to change to fibrous encapsulation (FC) (30 days-specimen, x40). (Candance A. Miranda and Dr. K. Muffly helped in tissue preparation and data collection respectively in Dr. K. Muffly's laboratory, USF College of Medicine) 
Another implant was an $a$-SiC coated $\mathrm{Ti} / \mathrm{Au}$ antenna, which is a well-known in vivo coating material. A thin $120 \mathrm{~nm}$ thick $a$-SiC film was deposited on $3 \mu \mathrm{m}$ thick $\mathrm{SiO}_{2}$ layer (on Ti/Au antenna) via PECVD [details described in 4.3.2]. The histopathological tissue analysis is shown in Figure 45. There was no sign of inflammatory response for 3 of the 4 implants. However tissue in contact with the last implant contained a lot of fibroblasts. Around 29 days of implantation, a significant inflammation/swelling was observed at this implant site. Upon removal, the implant was found to be broken into a couple pieces. Most likely when the implant broke into pieces, it irritated the site, the underlying oxide was exposed to the tissue, which based on the above results would certainly cause the type of inflammation. We also observed this inflammatory response using tissue analysis. Overall the rest of the implants/tissue responses had no inflammatory sign, as shown in Figure 45 . We can therefore conclude that $a$-SiC coatings are indeed suitable for in-vivo applications, as expected from the literature.

Implants comprised of a single crystal 3C-SiC film on front side of Si substrate, with $a$-SiC coated on the back side, were also implanted. No inflammatory response was noticed during the tissue histological examination on 3 different implanted samples. There was some scar evident on the forth tissue, which might be from tissue recovery after the surgery. Otherwise, the tissue response, shown in Figure 46, had no inflammatory signs compared to healthy tissue.

Finally the protein inhibitor poly ethylene glycol (PEG) was applied as a coating on the Ti/Au antenna and showed the expected result - no sign of inflammatory response for all the samples which is shown in Figure 47. 


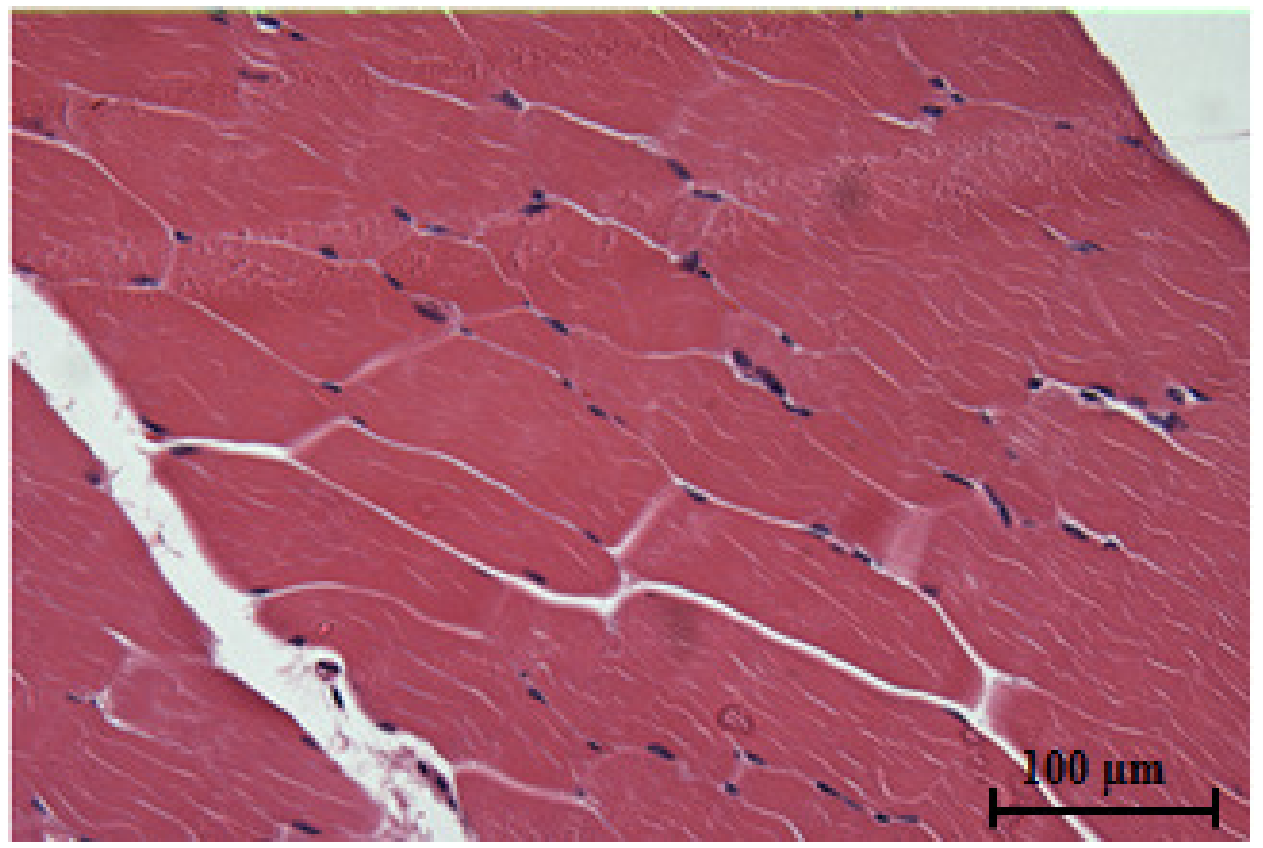

Figure 45: Histological tissue analysis of a-SiC coated $\mathrm{Ti} / \mathrm{Au}$ antenna. In general no sign of inflammation (30 days-specimen, x40). (Candance A. Miranda and Dr. K. Muffly helped in tissue preparation and data collection respectively in Dr. K. Muffly's laboratory, USF College of Medicine)

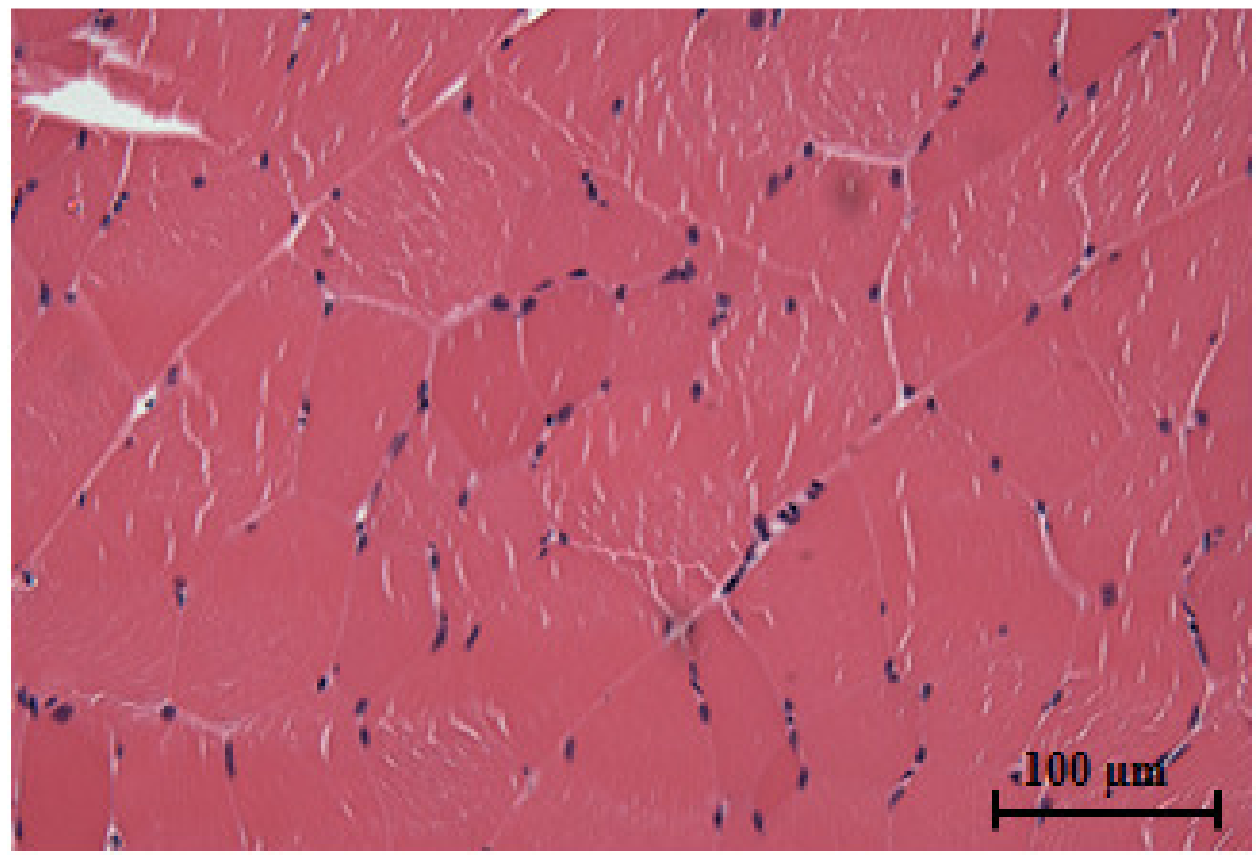

Figure 46: Histological tissue analysis of 3C- SiC/a-SiC coated sample. Overall no sign of inflammatory response (30 days-specimen, x40). (Candance A. Miranda and Dr. K. Muffly helped in tissue preparation and data collection respectively in Dr. K. Muffly's laboratory, USF College of Medicine). 


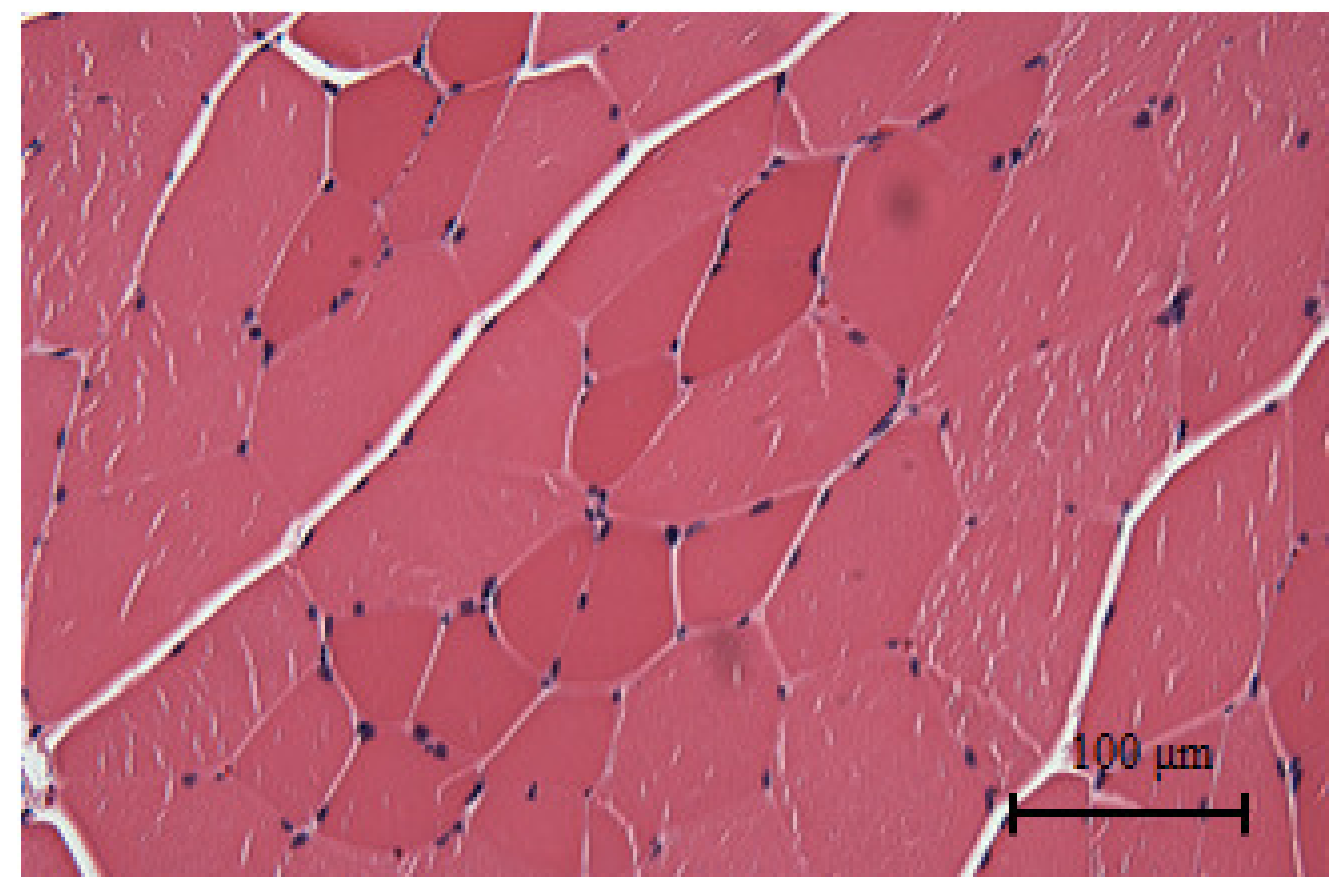

Figure 47: Histological tissue analysis of poly ethylene glycol (PEG) coated Ti/Au antenna. No sign of inflammatory response (30 days-specimens, x40). (Candance A. Miranda and Dr. K. Muffly helped in tissue preparation and data collection respectively in Dr. K. Muffly's laboratory, USF College of Medicine).

For this 30 day in vivo study, we found $\mathrm{Si}$ and $\mathrm{SiO} 2$ to have an inflammatory response with fibrous encapsulation development surrounding the implants, as expected. The bare Ti/Au antenna also had some inflammatory signs, even though there was no encapsulation. On the other hand, the PEG coated antenna and the all-SiC antenna did not have any inflammatory immune response whatsoever. At the same time, histological tissue analysis on single crystal $3 \mathrm{C}-\mathrm{SiC}$ and a-SiC coated samples did not show any significant inflammatory response. The overall tissue histological inflammatory response of all seven implants is shown in Figure 48. 


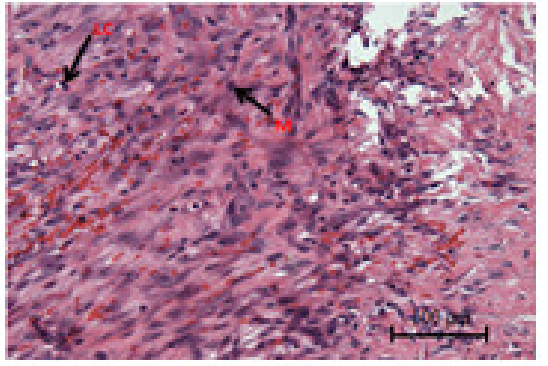

(a)

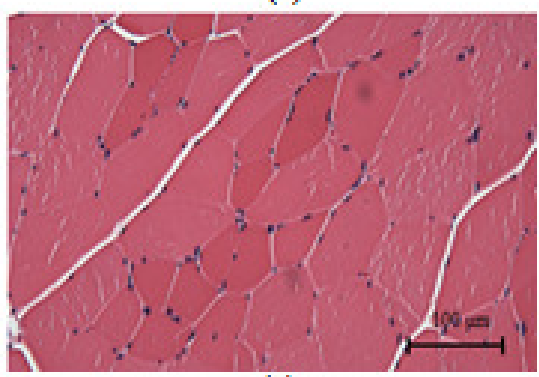

(c)

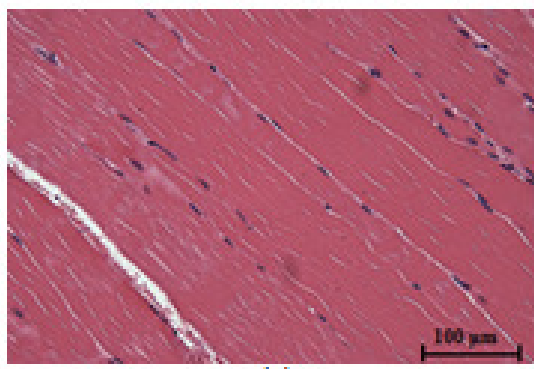

(e)

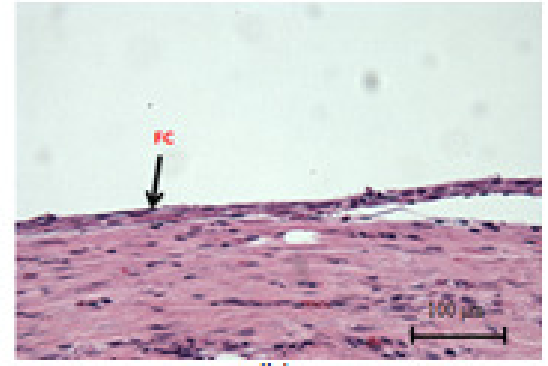

(b)

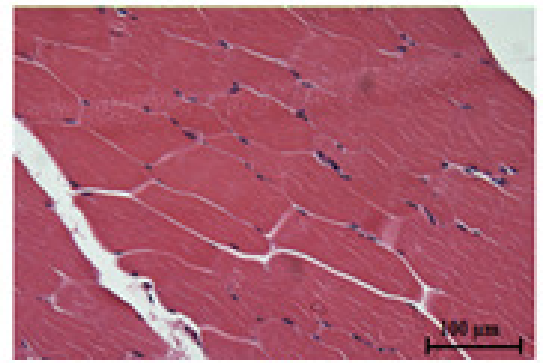

(d)

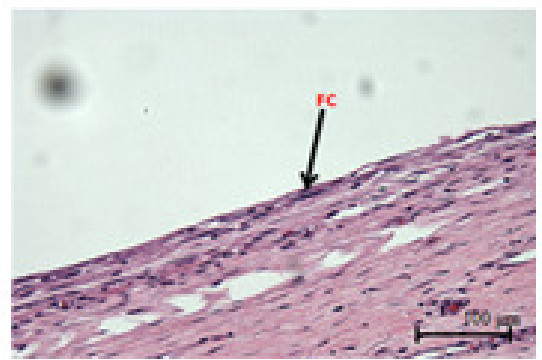

(f)

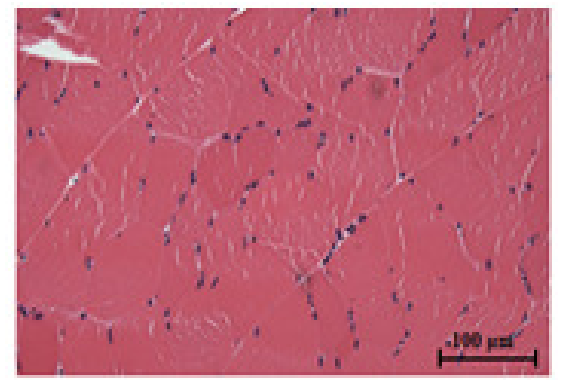

(g)

Figure 48: Seven different implant's histological tissue response. (a) Ti/Au antenna on SI $4 \mathrm{H}-\mathrm{SiC}$ as the base, (b)SiO2 coated $\mathrm{Ti} / \mathrm{Au}$ antenna on SI 4H-SiC, (c)PEG coated Ti/Au antenna on $\mathrm{SI} 4 \mathrm{H}-\mathrm{SiC}$, (d) Amorphous $\mathrm{SiC}$ (a-SiC) coated Ti/Au antenna on $\mathrm{SI} 4 \mathrm{H}-\mathrm{SiC}$, (e) all $\mathrm{SiC}$ antenna - poly $3 \mathrm{C}-\mathrm{SiC}$ on $\mathrm{SI} 4 \mathrm{H}-\mathrm{SiC}$, (f) Silicon ( $\mathrm{Si}$ ), (g) 3C-SiC and a-SiC coat on the back. 


\subsection{Results and Discussion}

In this chapter, an in vivo experiment for foreign body response to subcutaneouslyimplanted $\mathrm{SiC}$ based antennas has been conducted using a pig animal model. We have selected 5 different antennas for implantation and looked at the material interaction in vivo through any observed surface modifications of the implants as well as the tissue in proximity to the coatings. Started with 1) Ti/Au antenna on SI 4H-SiC as the base sensor; 2) $\mathrm{SiO}_{2}$ coated $\mathrm{Ti} / \mathrm{Au}$ antenna on $\mathrm{SI} 4 \mathrm{H}-\mathrm{SiC}$. One of the reasons for choosing this $\mathrm{SiO}_{2}$ coated sample is to reduce direct adhesion problems of other biocompatible material coatings such as $a-\mathrm{SiC}$ and poly ethylene glycol (PEG) on the Ti/Au antenna surface; 3) PEG coated $\mathrm{Ti} / \mathrm{Au}$ antenna on $\mathrm{SI} 4 \mathrm{H}-\mathrm{SiC}$. The bio-compatibility of covalently bonded Poly ethylene glycol (PEG) can remarkably reduce the non-specific adsorption of proteins and, therefore, enhance the biocompatibility; 4) biocompatible amorphous $\mathrm{SiC}$ (a-SiC) thin film coating of $\mathrm{Ti} / \mathrm{Au}$ antenna is another approach to make the antenna surface biocompatible; and 5) all $\mathrm{SiC}$ antenna, poly 3C-SiC on SI 4H-SiC.

After cleaning and disinfecting the devices, they were subcutaneously implanted for 30 days. The surface morphology was characterized before and after implantation using AFM. As SiC material is chemically inert, the surface roughness of 3C-SiC, $a$-SiC and poly $3 \mathrm{C}-\mathrm{SiC}$ had similar root mean square values before and after implantation. The roughness of $\mathrm{Au}, \mathrm{SiO}_{2}$ and $\mathrm{Si}$ were increased from before implantation to after implantation by $11 \mathrm{~nm}$ RMS, $26 \mathrm{~nm}$ RMS and $34.5 \mathrm{~nm}$ RMS, respectively. Tissue histology analysis of 30 day in vivo study showed that $\mathrm{Si}$ and $\mathrm{SiO}_{2}$ have an inflammatory response with fibrous encapsulation development surrounding the implants. Some inflammatory signs were found on bare $\mathrm{Ti} / \mathrm{Au}$ antenna, even though there was no 
encapsulation. On the other hand, the PEG coated antenna and the all-SiC antenna did not have any inflammatory immune response whatsoever. At the same time, histological tissue analysis on $a$-SiC coated and single crystal 3C-SiC samples did not show any significant inflammatory response. 


\section{CHAPTER 5}

\section{SUMMARY AND FUTURE WORK}

\subsection{Summary}

An antenna-based biocompatible implantable sensor for continuous glucose monitoring was realized using a $\mathrm{SiC}$ substrate with $\mathrm{Ti} / \mathrm{Au}$ metallization. The long-term goal is to develop an implantable antenna sensor operational for extended periods of time using the proven biocompatible material $\mathrm{SiC}$ for continuous glucose monitoring. Based on a review of the literature, we identified certain drawbacks of current implantable continuous glucose monitoring approaches which may be intolerable because of early biofouling, therefore a sensor based on a biocompatible material needed to be investigated. This work demonstrates certain design considerations to realize fully $\mathrm{SiC}$ antenna methodologies for the electromagnetic coupling approach. However, the most significant contributions lie within the areas of health care monitoring systems.

We began with a preliminary study, and selected a simple microstrip patch antenna because they are low profile, conformable to planar and nonplanar surfaces, simple, and inexpensive to manufacture. The antenna requirements for biomedical applications including the frequency range were also presented. But to minimize the size and associated substrate costs, the design frequency was selected to be well outside the medical bands at $10 \mathrm{GHz}$. A simulation of the patch structure using a highly conductive 
metal $(\mathrm{Ti} / \mathrm{Au})$ supports the hypothesis that a bio-compatible, heavily doped $\left(10^{20} \mathrm{~cm}^{-3}\right)$ SiC patch design would work as an antenna. HFSS Ansys software was used for simulation of the antenna performance such as return loss, radiation pattern, etc. To evaluate the initial performance of a $\mathrm{SiC}$ based antenna, a $\mathrm{Ti} / \mathrm{Au}$ antenna (as metal patch) was fabricated on a semi-insulating $4 \mathrm{H}-\mathrm{SiC}$ substrate to serve as a "baseline" antenna, which also provides a point of reference for the non-metallic, all-SiC antenna using a rectangular patch antenna mask. An input RF signal was provided by the VNA to function the sensor antenna. The fabricated $\mathrm{Ti} / \mathrm{Au}$ antenna's response is better in terms of targeted resonance frequency of $10 \mathrm{GHz}$ and return loss $\mathrm{S}_{11}$ as compared to the simulated data. Therefore, we have investigated three structures/approaches to realize an all-SiC antenna sensor. Here the antenna electrode, a heavily doped $\mathrm{SiC}$ top layer, is the most important part for the antenna to radiate. From the simulation result, a doping concentration of at least $5 \times 10^{19} \mathrm{~cm}^{-3}$ should give a reasonable antenna response. The options for dopant incorporation in $\mathrm{SiC}$ are either in-situ doping during growth of the material or ion-implantation and subsequent annealing after growth. For in-situ n-type doping, typically Nitrogen $\left(\mathrm{N}_{2}\right)$ and Ammonia $\left(\mathrm{NH}_{3}\right)$ gases are used.

The first approach is known as an etch back approach where $4 \mathrm{H}-\mathrm{SiC}$ semi-insulating wafers were used as substrates to grow highly doped $4 \mathrm{H} \mathrm{SiC}$ epitaxial layers using a CVD system where palladium-purified hydrogen, $\mathrm{H}_{2}$, used as the transport (carrier) gas; propane $\left(\mathrm{C}_{3} \mathrm{H}_{8}\right)$ - the carbon precursor; and a $10 \%$ silane $\left(\mathrm{SiH}_{4}\right)$ premixed in $90 \%$ hydrogen ballast $\left(\mathrm{H}_{2}\right)$ as the silicon precursor. For n-type doping, an additional precursor, nitrogen $\left(\mathrm{N}_{2}\right)$, was used to grow a heavily doped $\mathrm{SiC}$ antenna electrode. In this approach the best film, in terms of quality and doping concentration $\left(4.2 \times 10^{18} \mathrm{~cm}^{-3}\right)$, 
was achieved which is an order of magnitude lower than the simulated desired doping concentration needed for the antenna to radiate efficiently. Morphological degradation of the $4 \mathrm{H}-\mathrm{SiC}$ material was mostly due to the use of an on-axis $\mathrm{SI} 4 \mathrm{H}-\mathrm{SiC}$ substrate, which adversely affected the device result. Therefore, could not fabricate the all-SiC antenna using the heavily-doped layer as the metallic-like radiating patch.

The second approach, also known as an etch-back approach involved highly doped polycrystalline 3C-SiC (poly-SiC) epitaxial growth and patterning. The concept of realizing a radio frequency $(\mathrm{RF})$ sensor using a $\mathrm{SiC}$ antenna which includes a semiinsulating 4H-SiC substrate, PECVD deposited 0.5-5 $\mu \mathrm{m} \mathrm{SiO}_{2}$, a poly-SiC LPCVD deposited seed layer,and an n-type doped polycrystalline 3C-SiC layer. The same precursor gases were used to grow the highly doped poly-SiC antenna electrode using the same CVD reactor. The duration of the growth plateau was 1 hour and 40 minutes and yielded a $1.5 \mu \mathrm{m}$ thick poly $3 \mathrm{C}-\mathrm{SiC}$ film. The antenna was patterned using standard photolithography and DRIE each. After SIMS analysis and antenna simulation, the estimated doping concentration was $\sim 1 \times 10^{19} \mathrm{~cm}^{-3}$. After fabrication and measurement the antenna response was unfortunately observed to be quite lossy.

The third approach used a fully planar approach where the same semi-insulating $4 \mathrm{H}-$ $\mathrm{SiC}$ was used as the substrate. To realize the heavily doped antenna region (metal like conductivity) ion implantation was applied to the $\mathrm{SiC}$ material. SRIM-TRIM simulation software was used to simulate an ion implantation box type doping (Nitrogen doped) profile. The input implant energies were: 50, 100, 150 and $200 \mathrm{keV}$ and the ion doses were: $4 \times 10^{-14} / \mathrm{cm}^{2}, 8 \times 10^{-14} / \mathrm{cm}^{2}, 10^{15} / \mathrm{cm}^{2}$ and $10^{15} / \mathrm{cm}^{2}$ for the implant energies, respectively. The maximum doping concentration was in the range of $10^{20} \mathrm{~cm}^{-3}$. The 
backside of the antenna was also implanted to make an electrical ground plane. After patterning the antenna mask (Nickel) ion-implantation was applied on the substrate at a high enough energy to synthesize a degenerate $\mathrm{SiC}$ layer to form the antenna electrode structure. After removing the Ni mask, the same (CVD) reactor was used for the post ionimplantation annealing. This post implant annealing is very important to activate the dopants as well as to improve the surface degradation during high energy ionimplantation. For high temperature $\left(1550{ }^{\circ} \mathrm{C}\right)$ annealing, graphite caps were formed to protect the surface to specifically supress the sublimation of $\mathrm{Si}$ (melting temperature 1410 ${ }^{\circ} \mathrm{C}$ ) by spinning photoresist following a hard bake for 30 minutes in the CVD reactor at $1550^{\circ} \mathrm{C}$ and in an Ar ambient. After annealing the graphite cap was removed using an Oxygen Plasma Asher. Due to the performance it is possible that the dopants were only partially activated during the annealing process. The antenna response was measured using the same VNA and this all-SiC antenna was directly compared with the metal $(\mathrm{Ti} / \mathrm{Au})$ antenna. The resonance peak was comparable but the overall response was lossy for glucose sensing purposes. Therefore the $\mathrm{SiC}$ based $\mathrm{Ti} / \mathrm{Au}$ antenna was further evaluated to investigate the glucose sensing mechanism.

In an effort to better understand the sensing mechanism, we demonstrated the feasibility of the biocompatible implantable $\mathrm{SiC}$ based $\mathrm{Ti} / \mathrm{Au}$ antenna sensor for in vitro continuous glucose monitoring. The operational position of this implantable sensor was selected within a fatty tissue region which is in close proximity to a blood vessel. Therefore, fatty tissue and synthetic body fluid (SBF), or acellular human blood plasma mimicking liquids were synthesized for in vitro testing. The permittivity and conductivity of the liquids were measured using an HP 85070A dielectric probe kit. Different weights 
of D-glucose were added to the blood mimicking liquid in order to represent various glucose levels. The antenna sensor was tested via reflection coefficient measurements carried out by using the VNA. For in vitro blood glucose measurements, the antenna was placed inside the fatty layer in close proximity to the blood mimicking layer. The baseline reading from the sensor was taken by using blood mimicking liquid with zero glucose concentration. Following this, three blood mimicking liquids exhibiting different glucose concentrations were utilized within the experimental set-up to sequentially observe the variation in the resonance frequency of the antenna. The measured shift in the resonance frequency was towards lower frequency by as much as $40 \mathrm{MHz}$ for the largest glucose concentration of $530 \mathrm{mg} / \mathrm{dl}$. This implies a $97 \mathrm{kHz}$ shift per $1 \mathrm{mg} / \mathrm{dl}$ change in blood glucose. The simulated $S_{11}$ response of the antenna using the measured permittivity values of the fat and blood mimicking liquids was found to be in good agreement with the experimental results. The promising performance of the $\mathrm{SiC}$ based $\mathrm{Ti} / \mathrm{Au}$ glucose sensor using blood mimicking liquid led us to demonstrate the same sensor's performance using pig blood in vitro. Similarly, different weights of D-glucose were added to the pig blood in order to represent various glucose levels. The antenna sensor was also able to determine glucose concentration by observing a well-defined shift in the resonance frequency, and this was accomplished without any direct contact of the sensor with the blood. Using pig blood, a $62 \mathrm{kHz}$ resonance frequency shift per $1 \mathrm{mg} / \mathrm{dl}$ was observed. Both results showed the linearity of the response and usefulness of the biocompatible material- SiC-based sensor for long term, real -time continuous glucose monitoring for diabetic patients. 
A 30 day in vivo experiment for foreign body response to subcutaneously-implanted devices has been conducted using a pig/swine animal model. Total seven different implants - $\mathrm{Ti} / \mathrm{Au}$ antenna on $\mathrm{SI} 4 \mathrm{H}-\mathrm{SiC}$ as the base, $\mathrm{SiO} 2$ coated $\mathrm{Ti} / \mathrm{Au}$ antenna on $\mathrm{SI} 4 \mathrm{H}-$ $\mathrm{SiC}$, PEG coated $\mathrm{Ti} / \mathrm{Au}$ antenna on $\mathrm{SI} 4 \mathrm{H}-\mathrm{SiC}$, amorphous $\mathrm{SiC}(a-\mathrm{SiC})$ coated $\mathrm{Ti} / \mathrm{Au}$ antenna on $\mathrm{SI} 4 \mathrm{H}-\mathrm{SiC}$, all $\mathrm{SiC}$ antenna - poly $3 \mathrm{C}-\mathrm{SiC}$ on $\mathrm{SI} 4 \mathrm{H}-\mathrm{SiC}$, silicon ( $\mathrm{Si}), 3 \mathrm{C}-\mathrm{SiC}$ and $a-\mathrm{SiC}$ coat on the back were implanted. Tissue histology analysis showed that $\mathrm{Si}$ and $\mathrm{SiO}_{2}$ had an inflammatory response with fibrous encapsulation development surrounding the implants. Some inflammatory signs were found on bare Ti/Au antenna, even though there was no encapsulation. On the other hand, the PEG coated antenna and the all-SiC antenna did not have any inflammatory immune response whatsoever. At the same time, histological tissue analysis on $a$-SiC coated and single crystal 3C-SiC samples did not show any significant inflammatory response.

\subsection{Recommendations for Future Works}

To achieve the long term goal of developing an implantable antenna sensor operational for extended periods of time using fully biocompatible material $\mathrm{SiC}$ for continuous glucose monitoring, we plan to continue this research with strong emphasis on optimizing the antenna resonance frequency, growth of highly doped SiC epitaxial layers, wireless powering etc. The technical objectives are to: I) design a $2^{\text {nd }}$ generation SiC based implantable antenna sensor operational in the Medical Implant Communication Service (MICS) band, 402 to $405 \mathrm{MHz}$ or the Industrial, Scientific and Medical band (900 MHz, 2.4 GHz, 5.8 GHz); II) to realize an all SiC antenna sensor, the growth of insitu heavily doped $4 \mathrm{H}-\mathrm{SiC}$ homo epitaxial layer on off-axis $\mathrm{SI} 4 \mathrm{H}-\mathrm{SiC}$ substrate as an 
antenna electrode; and III) antenna sensor test in pig body with operational life time; estimate the link budget by electromagnetic wave propagation in lossy tissue/skin medium and perform a sensitivity analysis.

\subsubsection{Antenna Design Optimization}

The $\mathrm{SiC}$ based antenna sensor was designed in free space for $10 \mathrm{GHz}$ operation just to prove the sensor performance. As this sensor is promising for implantable continuous glucose monitoring, the sensor's resonance frequency has to be optimized for the MICS/ISM bands. Design of an implantable antenna is challenging in different ways. First the most important aspect is the antenna has to radiate into a complex lossy medium (the body). Second, the designed antenna has to be reasonably miniaturized. Another important optimization is the energy that needs to be transmitted by the implantable antenna out of the body to the receiver or vice versa. Depending on the substrate type, the classical antenna miniaturization strategies can also be applied to the implantable antenna such as lumped element loading, shorting the ground plane, etc. But as we are using hard SiC material we will not be able to use some of these techniques such as grounding through a substrate via, etc. Some potential antenna configurations are serpentine antennas, coplanar waveguide (CPW) antennas, fed slot antenna, etc.

After designing the optimum antenna, the link power budget can be calculated using the following formula [93]:

$$
\begin{gathered}
{[\text { Received power }=\text { transmitted power }+ \text { gain }- \text { losses }]} \\
{\left[P_{R_{x}}\right]_{d B m}=\left[P_{T_{x}}\right]_{d B m}+\left[G_{T_{x}}\right]_{d B}+\left[G_{R_{x}}\right]_{d B}-[\text { loss }]_{d B m}} \\
\text { where, }[\text { loss }]_{d B m}=[P L]_{d B m}+\left[e_{p}\right]_{d B m}+\left[M L_{T_{x}}\right]_{d B m}+\left[M L_{R_{x}}\right]_{d B m}
\end{gathered}
$$




$$
\text { and Path Loss, }[P L]_{d B m}=10 n \log \left(\frac{d}{d_{0}}\right)+10 \log \left(\frac{4 \pi d_{0}}{\pi_{0}}\right)^{2}+[s]_{d B}
$$

Here, $\left[P_{R_{x}}\right]_{d B m}$ is the power received by the antenna, $\left[P_{T_{x}}\right]_{d B m}$ is the power available at the transmitting antenna, $\left[G_{T_{\chi}}\right]_{d B}$ and $\left[G_{R_{x}}\right]_{d B}$ are the gains of transmitting and receiving antenna, respectively. Again, $\left[e_{p}\right]_{d B m}$ polarization mismatch factor, $\left[M L_{T_{x}}\right]_{d B m}$ and $\left[M L_{R_{x}}\right]_{d B m}$ the impedance mismatch loss of transmitting and receiving antenna. Now, $n$ is the path loss exponent, $d$ is the distance between the antennas, $s$ is the random scatter around the mean.

\subsubsection{Highly Doped Epilayer Optimization for all-SiC Antenna}

Among all-SiC antenna approaches, we were trying to grow a homoepitaxial heavily nitrogen doped epilayer on a SI-4H-SiC substrate in approach (a). Even after optimization, we did not grow a quality film. This morphological degradation of the $4 \mathrm{H}-$ $\mathrm{SiC}$ material was due to using an on-axis $\mathrm{SI} 4 \mathrm{H}-\mathrm{SiC}$ substrate. It has been realized previously that for high-quality epitaxial growth, off-axis substrates are needed due to the need to control the SiC polytype during growth [39]. Most of the boule/bulk materials are grown on-axis parallel to the c-axis and the wafers are sliced from the boule $4^{\circ}$ off-axis. As a result, off-axis $\mathrm{SI} 4 \mathrm{H}-\mathrm{SiC}$ are more expensive than on-axis material. Therefore, epitaxial growth on costly off-axis $\mathrm{SI} 4 \mathrm{H}-\mathrm{SiC}$ substrate should offer improvements not only morphologically but also in terms of hitting the require doping level of $10^{20} \mathrm{~cm}^{-3}$ [41]. To realize an all-SiC antenna, this approach would yield the best performance due to the high quality and heavily doped antenna electrode layer. 


\subsubsection{Antenna Sensor Test in Pig Body with Operational Life-time}

As mentioned earlier, the in vitro measurement of the glucose levels has been conducted using pig blood. The current antenna's resonance frequency was $10 \mathrm{GHz}$ which is a lot higher than the medical band frequency (MICS - $402 \mathrm{MHz}$ ). Even though we have implanted the antennas using an animal (pig) model for tissue histology analysis, we could not test the antenna performance at this frequency range. We will test the antenna sensor in vivo using pigs to address the efficacy and sensitivity of the sensor after optimization of the operating frequency (MICS/ISM band). We are also anticipating that we will be able to perform tests between three to six months to see the operational life time as well as biocompatibility of the sensor in vivo. 


\section{REFERENCES}

[1] Kumari TV, Vasudev U, Kumar A, Menon B. 'Cell surface interaction in the study of biocompatibility.' Trends Biomat Artif Organs, 15, 2002: 37-41.

[2] Wilkins, E. 'Toward implantable glucose sensors: a review.' J. Biomedical Engineering, 11, 1989: 353-361.

[3] Wilkins, E. 'A rechargeable glucose electrode long term in-vitro performances.' Biomed Instrum. \& Technol. 27, 1993: 325-333.

[4] Pickup, J.C., Glucose sensors and closed-loop insulin delivery. Biotechnology of Insulin Therapy, Blackwell, Oxford, 1991: 126-153.

[5] Pickup J.C., 'In-vivo glucose monitoring: sense and sensorobilitiy.' Diabetes Care, 16, 1993: 535-539.

[6] Cicero G, Catellani A., 'Towards SiC surface functionalization: An ab initio study.' J of Chem Phys, 2005;122:214716-1-5.

[7] Saddow SE, Agrawal A, editors, Advances in Silicon Carbide Processing and Applications, Norwood: Artech House; 2004.

[8] N. S. Oliver, C. Toumazou, A. E. G. Cass and D. G. Johnston, 'Review Article: Glucose sensors: a review of current and emerging technology' DIABETIC Medicine, 1464-5491, 2008.

[9] Tatara T, Tsuzaki K., 'Derivation of extracellular fluid volume fraction and equivalent dielectric constant of the cell membrane from dielectric properties of the human body' Med Biol Eng Comput 2000; 38: 377-83. J.

[10] Hayashi Y, Oshige I, Katsumoto Y, Omori S, Yasuda A, Asami K, 'Temporal variation of dielectric properties of preserved blood,' Phys Med Biol, 2008; 53: 295304.

[11] S. Kim, H. Melikyan, J. Kim, A. Babajanyan, J-Ha Lee, L. Enkhtur, B. Friedman, K. Lee, 'Noninvasive in vitro measurement of pig-blood D-glucose by using a microwave cavity sensor', Diabetes Research and Clinical Practice, 96,379-384, 2012. 
[12] Kim J, Babajanyan A, Hovsepyan A, Lee K, Friedman B., 'Microwave dielectric resonator biosensor for aqueous glucose solution,' Rev Sci Instrum, 2008;79:086107.

[13] Lee K, Babajanyan A, Kim Ch, Kim S, Friedman B., 'Glucose aqueous solution sensing by a near-field microwave microprobe,' Sensors and Actuators A $2008 ; 148: 28-32$.

[14] J.Venkataraman, B. Freer, 'Feasibility of Non-Invasive Blood Glucose Monitoring', 2011 IEEE International Symposium on Antennas and Propagation and USNC/URSI, 603-6062011.

[15] Pickup J.C., Claremont DJ, Shaw GW, 'Responses and calibration of amperometric glucose sensors implanted in the subcutaneous tissue of man', Acta Diabeto, l 1993, 30:143-148.

[16] Thome-Duret V, Gangnerau MN, Zhang Y, Wilson GS, Reach G, 'Modification of the sensitivity of the glucose sensor implanted into subcutaneous tissue,' Diabetes Metab, 22; 1996.

[17] Robert D. Black, 'Recent Advances in Translational Work on Implantable Sensors,' IEEE SENSORS JOURNAL, 11, No. 12, 2011.

[18] X. Li, X. Wang, R. Bondokov, J. Morris, Y. H. An, and T. S. Sudarshan, 'Micro/nanoscale mechanical and tribological characterization of SiC for orthopedic applications,' J. Biomed. Mater. Res. B Appl. Biomater., 72, 2, 353 - 361, 2005.

[19] U. Kalnins, A. Erglis, I. Dinne, I. Kumsars, and S. Jegere, 'Clinical outcomes of silicon carbide coated stents in patient with coronary diseas,' Med. Sci. Monit., $2,16-20,2002$.

[20] S. Santavirta, M. Takagi, L. Nordsletten, A. Anttila, R. Lappalainen, and Y. T. Konttinen, 'Biocompatibility of silicon carbide in colony formation test in vitro: A promising new ceramic THR implant coating material', Arch. Orthop. Trauma Surg., $118,1-2,89-91,1998$.

[21] J-M. Hsua, P. Tathireddy, L. Rieth, A. R. Normann, and F. Solzbacher, 'Characterization of a-SiCx:H thin films as an encapsulation material for integrated silicon based neural interface devices', Thin Solid Films, 516, 1, 34 - 41, 2007.

[22] S.E. Saddow, Editor, Silicon Carbide Biotechnology: A Biocompatible Semiconductor for Advanced Biomedical Devices and Applications, (C) 2011 Elsevier, UK.

[23] A. Naji and M. Harmand, 'Cytocompatibility of two coating materials, amorphous alumina and silicon carbide, using human differentiated cell cultures', Biomaterials, 12, 7, $690-694,1991$. 
[24] R. Yakimova, R. M. Petoral Jr, R. M. Yazdi, G. R. Vahlberg, C. L. Spetz, and A. K. Uvdal, 'Surface functionalization and biomedical applications based on SiC', $J$. Phys. D: Appl. Phys., 40, 6435 - 6442, October 2007.

[25] C. A. Zorman, M. Mehregany, Materials for microelectromechanical systems, in The MEMS Handbook: Introduction and Fundamentals, M. Gad-el-Hak, Ed., Boca Raton, FL, USA:CRC Press, 2001, pp. 15 - 23.

[26] M E. Levinshtein, S. L. Rumyantsev, M. S. Shur, Eds., Properties of Advanced Semiconductor Materials, New York, NY, USA: John Wiley and Sons, 2000.

[27] J. Deva Reddy, A. A. Volinsky, C. Frewin, C. Locke, and S. E. Saddow, 'Mechanical properties of single and polycrystalline SiC thin films,' in Mat. Res. Soc. Symp. Proc., 1049, 2008, AA03 - AA06.

[28] X. Li, X. Wang et al., 'Micro/Nanoscale Mechanical and Tribological Characterization of SiC for Orthopedic Applications', J Biomed Mater Res B Appl Biomater., Feb 15; 72, (2), 353-61 (2005).

[29] A.J. Rosenbloom, D.M. Sipe, Y. Shishkin, Y. Ke, R.P. Devaty and W.J. Choyke, Nanoporous SiC: "A Candidate Semi-Permeable Material for Biomedical Applications", Biomedical Microdevices, 6, (4), 261-267, 2004.

[30] Coletti C, Jaroszeski, MJ, Pallaoro A, Hoff AM, Iannotta S, Saddow, SE., 'Biocompatibility and wettability of crystalline $\mathrm{SiC}$ and $\mathrm{Si}$ surfaces', Proceedings of the 29th International conference of the IEEE EMBS; 2007 Aug 23-26; Lyon, France.p.5849-52.

[31] Voskerician, G., Shive MS, Shawgo RS, Von Recum H, Anderson JM, Cima MJ, Langer R., 'Biocompatibility and biofouling of MEMS drug delivery devices.' Biomaterials, 2003;24:1959-67.

[32] S.E. Saddow, C. Coletti, C.L. Frewin, N. Schettini, A. Oliveros and M. Jarosezeski, 'Single-crystal Silicon Carbide: A Biocompatible and Hemocompatible', MRS Proceedings, 1246, 193-198 2010.

[33] Stutzman, W.L. and Thiele, G.A., Antenna Theory and Design, John Wiley \& Sons, Inc, 1998.

[34] Balanis, Contantine A., Antenna Theory, Analysis and Design, Third Edition, 2005: ISBN: 0-471-66782-X.

[35] Pnescu, Dorin, 'Emerging Technologies [wireless communication systems for implantable medical devices]', Engineering in Medicine and Biological Magazine, IEEE, 27, 2, March-April 2008. 
[36] Michael Shur, Introduction to Electronic Devices, ISBN 0-471-10348-9, 1995.

[37] Mulpuri V. Rao, Jesse B. Tucker, M. C. Ridgway, O. W. Holland, N. Papanicolaou, 'Ion-implantation in bulk semi-insulating 4H-SiC', J. Appl. Phys. 86, 752 (1999).

[38] Christopher Locke, Growth of 3C-SiC on (111) Si using hot-wall chemical vapor deposition, MS Thesis, University of South Florida, 2009.

[39] Sivaram S. 'Chemical Vapor deposition: thermal and plasma deposition electronic materials'. Van Nostrand Reinhold, Newyork, 1995.

[40] R. Myers-Ward, High Growth Rate SiC CVD via Hot-Wall Epitaxy, PhD. Dissertation, University of South Florida (c) 2006.

[41] H. K. Song , H. S. Seo, S. Y. Kwon, J. H. Moon, J. H. Yim, J. H. Lee, H. J. Kim, 'Heavily nitrogen-doped $4 \mathrm{H} \mathrm{SiC}$ homoepitaxial films grown on porous $\mathrm{SiC}$ substrates', Journal of Crystal Growth, 305, 83-87, 2007.

[42] C.L. Frewin, C. Locke, J. Wang, P. Spagnol and S.E. Saddow, 'Growth of cubic silicon carbide on oxide using polysilicon as a seed layer for micro-electromechanical machine applications', Journal of Crystal Growth, 311, 17, 4179-4182 (2009).

[43] R. L. Myers, Y. Shishkin, O. Kordina, and S. E. Saddow, 'High growth rates $(>30 \mu \mathrm{m} / \mathrm{h})$ of $4 \mathrm{H}-\mathrm{SiC}$ epitaxial layers using a horizontal hot-wall CVD reactor', $J$. Cryst. Growth, 285 (2005) 483-486.

[44] Shamima Afroz, Sylvia W Thomas, Gokhan Mumcu, Christopher W. Locke, Stephen E. Saddow, 'A Biocompatible SiC RF Antenna for In-Vivo Sensing Applications', Conference proceedings, MRS Spring Meeting proceedings, SanFrancisco, USA, April 9-13, 2012

[45] A. U. Alahakone and S. Senanayake, 'A real-time system with assistive feedback for postural control in rehabilitation,' IEEE/ASME Trans. Mechatronics, 15, 2, 226233, Apr. 2010.

[46] K. D. Nguyen, I. M. Chen, Z. Q. Luo, S. H. Yeo, and H. B. L. Duh, 'A wearable sensing system for tracking and monitoring of functional arm movement,' IEEE/ASME Trans. Mechatronics, 16, 2, 213-220, Apr. 2011.

[47] O. Aziz, L. Atallah, B. Lo, M. ElHelw, L.Wang, Z. G. Yang, and A. Darzi, 'A pervasive body sensor network for measuring postoperative recovery at home,' Surg. Innov., 14, 83-90, Jun. 2007. 
[48] Schwan HP., 'Electrode polarization impedance and measurements in biological materials', Ann. N.Y. Acad. Sci. 1968:148-191.

[49] S. C. Hagness, A. Taflove, and J. E. Bridges, 'Wideband Ultra-Low Reverberation Antenna for Biological Sensing,' Electron. Lett., 33, 1594-1595, Sept. $11,1997$.

[50] Hayashi Y, Livshits L, Caduff A, Feldman Y, 'Dielectric spectroscopy study of specific glucose influence on human erythrocyte membranes', $J$ Phys D 2003; 36: 369-74.

[51] Berg JM, Tymoczko JL, Stryer L. Biochemistry, 5th edition, New York: W H Freeman, 2002.

[52] L Livshits, A Caduff, M S Talary and Y Feldman, 'Dielectric response of biconcave erythrocyte membranes to D- and L-Glucose', Journal of Physics, 40, 9$15,2007$.

[53] E. Topsakal, T. Karacolak, and E. C. Moreland, 'Glucose-Dependent Dielectric Properties of Blood Plasma', IEEE International Symposium on Antennas and Propagation and USNC/URSI, 2011.

[54] University of Michigan Medical Library.

[55] P.Soontornpipit, C.M. Furse, and Y.C. Chung, 'Design of implantable microstrip antennas for communication with medical implants,' IEEE Trans Microw Theory Tech. 52, 8, 1944-1951, Aug, 2004.

[56] J.Kim and Y. Rahmat-Samii, 'Implanted antennas inside a human body: Simulations, designs, and characterizations,' IEEE Trans. Microw Theory Tech, 52, 8, 1934-1943, Aug, 2004.

[57] K.Gosalia, M.S. Humayun, and G.Lazzi, 'Impedance matching and implementation of planar space-filling dipoles as intraocular implanted anrennas in a retinal prosthesis,' IEEE Trans. Antennas Propag, 53, 8, 2365-2373, Aug, 2005.

[58] S.Jalota, S.B.Bhaduri, and A. Cuneyt Tas, 'In-vitro testing of Calcium Phosphate Whiskers', Wiley Inter Science, May 2008.

[59] Bayraktar, A.C. Tas, 'Chemical preparation of Carbonated Calcium Hydroxyapatite powders at $370 \mathrm{C}$ Urea-containing synthetic body fluids,' J. Eur. Ceram. Soc.19 (1999).

[60] TRUE track Blood Glucose Monitoring.

[61] C. Gabriel, S. Gabriel, E. Courthout, 'The dielectric properties of biological tissues: I Literature survey,' Phys. Med. Biol. 41 (1996) 2231-2249. 
[62] Williams, D. F. ed., Definitions in Biomaterials, Elsevier: Amsterdam, 1987; p 72.

[63] Wikipedia http://en.wikipedia.org/wiki/Biomaterial, accessed 9 Feb 2013.

[64] Collings, A. F.; Caruso, F. Report on Progress in Physics, 1997, 60, (11), 13971445.

[65] Mar, M. N.; Ratner, B. D.; Yee, S. S. 'An intrinsically protein-resistant surface plasmon resonance biosensor based upon a RF-plasma-deposited thin film', Sensors and Actuators B-Chemical, 1999, 54 (1-2), 125-131.

[66] Metzger, S. W.; Natesan, M.; Yanavich, C.; Schneider, J.; Lee, G. U., 'Development and characterization of surface chemistries for micro-fabricated biosensors', Journal of Vacuum Science \& Technology A. Surface Films, 1999, 17 (5), 2623-2628.

[67] Masson, J. F.; Battaglia, T. M.; Cramer, J.; Beaudoin, S.; Sierks, M.; Booksh, K. S., 'Reduction of nonspecific protein binding on surface plasmon resonance biosensors', Analytical and Bioanalytical Chemistry, 2006, 386, (7-8), 1951-1959.

[68] Strehlitz, B.; Nikolaus, N.; Stoltenburg, R., 'Protein Detection with Aptamer Biosensors', Sensors, 2008, 8, (7), 4296-4307.

[69] W. Kenneth Ward, 'A Review of the Foreign body Response to Subcutaneouslyimplanted Devices: The Role of Macrophages and Cytokines in Biofouling and Fibrosis', Journal of Diabetes Science and Technology, 2, 5, Sep. 2008.

[70] Sharkaway AA, Klitzman B, Truskey GA, Reichert WM, 'Engineering the tissue which encapsulates subcutaneous implants. I. Diffusion properties,' J. Biomed Materials Research, 1997; 37: 401-412.

[71] Sharkaway AA, Klitzman B, Truskey GA, Reichert WM, 'Engineering the tissue which encapsulates subcutaneous implants. II. Plasma-tissue exchange properties,' $J$. Biomed Material Research, 1998; 40: 586-597.

[72] Sharkaway AA, Klitzman B, Truskey GA, Reichert WM, 'Engineering the tissue which encapsulates subcutaneous implants. III. Effective tissue response times', $J$. Biomed Material Research, 1998; 40: 598-605.

[73] Ratner, B. D.; Bryant, S. J. Annu., 'BIOMATERIALS: Where We Have Been and Where We are Going', Annual Review of Biomedical Engineering, 2004, 6, 41-75.

[74] Hench, L. L.; Wilson, J., 'Surface-active biomaterials', Science, 1984, 226, (4675), 630-636. 
[75] Andrade, J. D.; Hlady V., 'Protein adsorption and materials biocompatibility: A tutorial review and suggested hypotheses', Advances in Polymer Science, 1986, 79, 163.

[76] Gristina, A. G., 'Biomaterial-centered infection: Microbial adhesion versus tissue integration', Science, 1987, 237, (4822), 1588-1595.

[77] Quake, S. R.; Scherer, A., 'From micro- to nanofabrication with soft materials', Science, 2000, 290, (5496), 1536-1540.

[78] Ostuni, E.; Chapman, R. G.; Liang, M. N.; Meluleni, G.; Pier, G.; Ingber, D. E.; Whitesides, G. M., 'Self-assembled monolayers that resist the adsorption of proteins and adhesion of bacterial and mammalian cells', Langmuir, 2001, 17, (20), 63366343.

[79] Bailey, F. E.; Koleske, J. V. Alkylene, Oxides and Their Polymers, Marcel Dekker Inc: New York, 1991; 35, p 272.

[80] Harris, J. M. ed. Poly (Ethylene Glycol) Chemistry: Biotechnical and Biomedical Applications. Plenum Press: New York, 1992; p 485.

[81] Morra, M., 'On the molecular basis of fouling resistance', Journal of Biomaterials Science, Polymer Edition, 2000, 11, (6), 547-569.

[82] Alexander, S., 'Polymer adsorption on small spheres. A scaling approach', Journal of Physics, 1977, 38, (8), 977-981.

[83] Degennes, P. G., 'Polymer solutions near an interface. Adsorption and depletion layers', Macromolecules, 1981, 14, (6), 1637-1644.

[84] Degennes, P. G., 'Polymers at an interface. 2. Interaction between two plates carrying adsorbed polymer layers', Macromolecules, 1982, 15, (2), 492-500.

[85] Jeon, S. I.; Andrade, J. D., 'Protein surface interactions in the presence of polyethylene oxide. 2. Effect of protein size', Journal of Colloid Interface Science, 1991, 142, (1), 159-166.

[86] Jeon, S. I.; Lee, J. H.; Andrade, J. D.; Degennes, P. G., 'Protein surface interactions in the presence of polyethylene oxide. I. Simplified theory', Journal of Colloid Interface Science, 1991, 142, (1), 149-158.

[87] Szleifer, I., 'Statistical thermodynamics of polymers near surfaces', Current Opinion in Colloid \& Interface Science, 1996, 1, (3), 416-423.

[88] Szleifer, I., 'Polymers and proteins: interactions at interfaces', Current Opinion in Colloid \& Interface Science, 1997, 2, (3), 337-344. 
[89] Halperin, A., 'Polymer Brushes that Resist Adsorption of Model Proteins: Design Parameters', Langmuir, 1999, 15, (7), 2525-2533.

[90] Besseling, N. A. M.; Lyklema, 'Equilibrium Properties of Water and Its LiquidVapor Interface', J. J. Phys. Chem. 1994, 98, (44), 11610-11622.

[91] Besseling, N. A. M.; Lyklema, 'Hydrophobic hydration of small apolar molecules.and extended surfaces: A molecular model', J. Pure Appl. Chem. 1995, 67, (6), 881-888.

[92] Vanoss, C. J.; Good, R. J.; Chaudhury, M. K., 'The role of van der Waals force and Hydrogen bonds in "Hydrophobic Interactions" between biopolymers and low energy surfaces', J. Colloid Interface Sci. 1986, 111, (2), 378-390.

[93] Asimina Kiourti, Konstantina S. Nikita, 'Miniature Scalp-Implantable Antennas for Telemetry in the MICS and ISM Bands: Design, Safety Considerations and Link Budget Analysis', IEEE Transactions on antennas and propagation, 60, 8, August, 2012. 


\section{APPENDIX 1: COPY RIGHT}

\section{PERMISSION INVOICE}

Inv. \# P03J 22629

March 7, 2013

Shamima Afroz

4202 E. Fawler Ave, Electrical Eng. Dept.

Universitiy of South Florida, ENB 118

Tampa, FL

33620

\section{REFERENCE ISSN: EISSN:}

Journal: $\quad$ Materials Research Society Symposia Proceedings, Vol. 1246, 2010

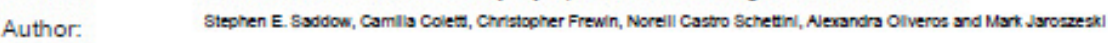

Tite:

Selection/pp.:

A Biocompatible and Hemocompatible Semiconductor for Advanced Biomedical Applications

Figure 1 and figure 2

Additional

Copyright $\odot 2010$ Materials Research Society. Reprinted with the permission of Cambridge University Press.

USE

Reprint Title: A Biocompatible SiC RF Antenna for In-vivo Sensing Applications

Publisher: Universitiy of South Florida

Format: dissertation / thesis

Quantity (Limit'): 100

Avail. Date: 2013

\section{RIGHTS/ACKNOWLEDGEMENT}

Permission is granted for nonexclusive rights throughout the World in the English language for interior text editorial use in the format described above only. Please fully acknowledge our material and indicate the copyright notice as it appears in our publication, followed by the phrase "Reprinted with the permission of Cambridge University Press."

All requests from third parties to reproduce this material must be forwarded to Cambridge University Press.

\section{FEES/RESTRICTIONS}

\section{$\$ 0.00$}

"This permission is restricted to the indicated format and quantity; for additional use, you must reapply for permission. This permission does not allow reprinting of any material copyrighted by or credited in our publication to another source; Cambridge disclaims all liability in connection with the use of such material without proper consent. A COPY OF THIS INVOICE MUST ACCOMPANY PAYMENT. Payment is due upon receipt of invoice. Terms: Net 60 days. Make check payable to Cambridge University Press, Attn: Rights and Permissions. (Fed. I.D. \#: 13-1599108.)

This permission does not supersede permission that may be required from the original source indicated in our publication.

This permission requires that you send zero $(0) \quad$ copies of your publication directly to our author and zero $(0)$ copy of your publication to this office upon availability.

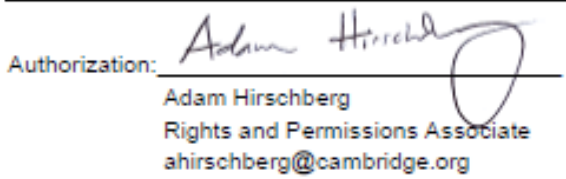




\section{APPENDIX 2: IACUC PROTOCOL AND APPLICATION}

\begin{tabular}{ll}
\hline \hline & \\
\hline MEMORANDUM & Lennox Hoyte, MD,MSEECS \\
TO: & Dept. of Obstetrics \& Gynecology \\
& STC-6 \\
INSTITUTIONAL ANIMAL CARE_USE COMMITIEE
\end{tabular}

The Institutional Animal Care and Use Committee (IACUC) reviewed your application requesting the use of animals in research for the above-entitled study. The IACUC requested modifications/further information in response to that review and has received the required information. The IACUC APPROVED your request to use the following animals in your protocol for a one-year period beginning $9 / 26 / 2012$ :

- $4 \mathrm{Pig}, \mathrm{male} / \mathrm{female}$

Please reference the above IACUC protocol number in all correspondence regarding this project with the IACUC, Comparative Medicine, or the Division of Research Integrity and Compliance. In addition, please take note of the following:

- IACUC approval is granted for a one-year period at the end of which, an annual renewal form must be submitted for years two (2) and three (3) of the protocol. After three years all continuing studies must be completely re-described in a new application and submitted to IACUC for review.

- All Comparative Medicine pre-performance safety and logistic meetings must occur prior to implementation of this protocol [IACUC policy V.10]. Please contact the program coordinator at compmed@research.usf.edu to schedule a pre-performance meeting.

- All changes to the IACUC-Approved Protocol must be pre-approved by the IACUC [IACUC policyIII.11]. Minor changes can be submitted to the IACUC for review and approval as an amendment or procedural change, whereas major changes to the protocol require submission of a new IACUC application. Minor changes are changes considered to be within the scope of the original research hypothesis or involve the original species and are submitted to the IACUC as an Amendment or Procedural change. Any change in the IACUC-approved protocol that does not meet the latter definition is considered a major protocol change and requires th submission of a new application. More information on what constitutes a minor versus major protocol change and procedural steps necessary for IACUC review and approval are available on the Comparative Medicine web site at http://www.research.usf.edu/cm/amendments.htm

- All costs invoiced to a grant account must be allocable to the purpose of the grant [IACUC policies IV.5 and V.10]. Costs allocable to one protocol may not be shifted to another in order to meet deficiencies caused by overruns, or for other reasons of convenience. Rotation of charges among protocols by month without establishing that the rotation schedule credibly reflects the relative benefit to each protocol is unacceptable.

For more information on IACUC policies and procedures, please visit the Comparative Medicine web site at http://www.research.usf.edu/cm/default.htm.

cc: Comparative Medicine

Division of Research Grants

OFFICE OF RESEARCH - DIVISION OF RESEARCH INTEGRITY AND COMPLIANCE

INSTITUTIONAL ANIMAL CARE AND USE COMMITTEE

PHS No. A4100-01, AAALAC No.58-15, USDA No. 58-15

University of South Florida - 12901 Bruce B. Downs Blvd., MDC35 - Tampa, FL 33612-4799

(813) 974-7106 · FAX (813) 974-709 


\section{APPENDIX 2- CONTINUED}

\section{APPLICATION FOR THE USE OF ANIMALS IN RESEARCH}

Submit an electronic version to CompMed@research.usf.edu for veterinary pre-review prior to Submission to the University of South Florida, Institutional Animal Care \& Use Committee, (Type all responses) MDC 35, phone 974-7106, fax 974-7091, email IACUC.@research.usf.edu

\section{TITLE OF PROJECT} PHS \#A4100-01; USDA \#58-R-0015; AAALAC \#000434

Long term implantable Silicon Carbide (SiC) RF antenna blood glucose sensor

(Note: The title of this IACUC application must match the title of the grant that supports this research. If the described activity involving animals is sup ported by multiple grants with different titles, or a grant is awarded later, during the 3-year approval period of this protocol, that supports this research, inform the IACUC by completing and submitting a Request to Amend an Animal Use Protocol form.)

\section{PERSONNEL}

Lennox Hoyte MD

Principal Investigator (including degrees)

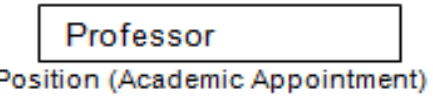

2 Tampa General

Campus Address

Karl E. Muffly PhD
Ihovte@health.usf.e

PI Email Address

kmuffly@health.usf.

Secondary Study Contact Email Address

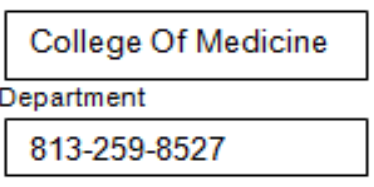

PI Phone

813-974-9385

Secondary Study Contact Phone

\section{QUALIFICATIONS}

\begin{tabular}{||l|l|l|l||}
\hline Name \& Degree & $\begin{array}{l}\text { Rol } \\
\text { e }\end{array}$ & Years Experience/Species & Cert\# \\
\hline Lennox Hoyte MD, MSEECS & PI & 6 years / pig & 3695 \\
\hline Karl E. Muffly, Ph.D. & SI & 4 years / pig & 202 \\
\hline Sylvia W. Thomas, Ph.D. & T & $0 /$ pig & 3344 \\
\hline Shamima Afroz, M.S. & T & $0 /$ pig & 6917 \\
\hline & & & \\
\hline & & & 1 \\
\hline
\end{tabular}




\section{APPENDIX 2- CONTINUED}

(Note: Indicate role of involved personnel as either Principal (PI), or Secondary (SI) Investigators, or technicians/assistants (T). Indicate each individual's years of experience with species described herein (e.g., $6 \mathrm{yrs} / \mathrm{mice}$, $4 \mathrm{yrs} / \mathrm{rabbits}$ ). Certification of orientation, training, and experience regarding the regulatory, occupational health and safety, and care and use as pects of the species requested herein is required prior to IACUC review of this protocol, and is available from the IACUC c/o the Division of Comparative Medicine, 974-9796. Certification numbers for each involved individual must be listed.)

\section{FUNDING SOURCE}

(Indicate funding agency, grant \#, and account \# if known.)

Agency: USF Department of ElectricalEngineering Grant \#:

5. LOCATION This protocol will be conducted at (please check location(s)): NOTE - Animal removal/relocation from a facility requires PRIOR APPROVAL.

\begin{tabular}{|c|c|c|c|c|c|c|c|c|c|c|c|c|}
\hline $\begin{array}{l}\text { AL } \\
Z\end{array}$ & \begin{tabular}{|l} 
CA \\
ML \\
\end{tabular} & $\begin{array}{l}\mathrm{C} \\
\mathrm{O} \\
\mathrm{M}\end{array}$ & $\mathrm{CPH}$ & CRI & IDRB & KRC & $\begin{array}{l}\text { I } \\
\text { S } \\
\text { A }\end{array}$ & NCF & PSY & SCA & SRB & $\begin{array}{l}\text { V } \\
\text { A } \\
\text { H }\end{array}$ \\
\hline & & $\mathbf{X}$ & & & & & & & & & & \\
\hline
\end{tabular}

Signature of Principal Investigator

\section{CERTIFICATION}

A search for alternatives and alternative methods, including a search of at least two relevant databases (e.g., PubMed, SCIRUS) indicated below, covering the indicated years (at least the last 10 yrs.), and using the indicated search term(s), demonstrates that suitable alternatives to these procedures, and to aspects of these procedures which may cause pain or discomfort to animals, or to this animal use are not available or applicable. The methods described will be used and continuously refined so as to reduce animal discomfort and use. Conduct will be in accordance with the PHS policy, AWR, Guide, AAALAC guidelines, DEA regulations, and IACUC Principles and Procedures. This project was designed with the consultation of a veterinarian. The described animal use does not duplicate previous or existing studies. This description is complete and accurate. Implementing changes to this description requires prior written IACUC approval. Current biomedical supplies will be used. Complete animal procedural/surgical/testing records will be maintained. Personnel are certified as adequately trained and experienced, and have complied with IACUC occupational health \& safety procedures. Comparative Medicine will be notified whenever data from any pre-clinical study involving animals is submitted to the Food \& Drug Administration.

Identify $\geq 2$ Databases Years: Search Sic antenna sensor cell $/$ surface

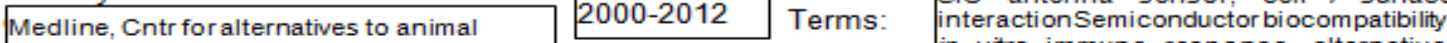
testing.U. of Calif. Cntrfor Animal Alternatives, Fund for the Rep of Animals in swine, surgery, analgesia, pain, early endpoints, pathology, and euthanasia

Note: Addended/added pages of response permissible, if they are identified as a response to which item \# and page \#. 


\section{APPENDIX 2- CONTINUED}

\section{PROTOCOL CLASSIFICATION}

7. Please check one.

a. $\mathbf{X}$ New Project; Proceed to Item \#8.

3rd Yr renewal replacing previous approved IACUC Protocol \#

b. Please provide a brief summary (a few sentences) describing work accomplished during the last approval period and how the work proposed in this renewal extends the previous studies.

\section{JUSTIFICATION FOR THE USE OF ANIMALS}

8. Briefly state in lay terms the purpose and scope of work of this request (i.e., research hypothesis or teaching objectives). (Note: Your response must be in language that a high school senior can understand.)

Many of the problems caused by a blood disorder, such as diabetes, can be minimized if blood sugar levels could be tightly controlled. Tight control of blood sugar can be obtained if blood sugar levels could be monitored continuously. At the present time, the best way to continuously monitor blood sugar levels is to surgically put into the body (implanted) a device that senses (sensor) sugar in the body. The problem is when a sensor is put into the body, sometimes the body will scar and swell. This scarring and swelling of the tissue may cover the sensor and make it difficult for the sensor to work properly. For this reason, currently available sensors tend to stop working after about 10 to 30 days following implantation. Our group has developed a blood sugar sensor, using a special material that resists the scarring and swelling that can cover the implanted sensor. It is believed that this new sensor will be able to monitor blood sugar levels for much longer periods of time compared to previous sensor designs. Previously, our group has shown that this new sensor material will not harm samples of skin or artificial laboratory tissue. However, testing of the new special material, implanted in the body of an animal, is required to prove that the new material will not be harmful in living tissues. The present study will find out how living tissue reacts to the new material. In addition, the study will test levels of living tissue scarring and levels of swelling. These tests will be completed after the new blood sugar sensor has been implanted for an extended period. Several designs of the sensor will be implanted, and the tissue reaction to each will be studied, after 30 days of implantation, using standard analysis methods.

9. Briefly outline or describe the procedures in which animals will be used (i.e., the general sequence and schedule of what will be done to the animals). Section 9 or Section 11 of the application should contain an explanation/rationale for assigning the specific number of animals to the different Pain Categories in Section 10.

(Note: For complicated experimental designs, it may be helpful to the IACUC if this response includes a flow chart, diagram, timeline, or table which depicts the experiments or sequence of events.)

After cleaning and disinfecting the implantable devices, they are ready for subcutaneous implantation. We select an adult pig of 20-35 Kg weight. Prior to induction of anesthesia, the pig will be fasted for 12 hours. Carprofen will be administered subcutaneously at the dose rate of $4.0 \mathrm{mg} / \mathrm{kg}$. Also prior to induction of general inhalational anesthesia, the animals will receive diazepam (at the initial dose rate of $4.0 \mathrm{mg} / \mathrm{kg}$ body weight PO) and atropine sulfate (at the dose rate of $0.05 \mathrm{mg} / \mathrm{kg} \mathrm{IM}$ ). Ketamine will be administered IM at the dose rate of $20 \mathrm{mg} / \mathrm{kg}$ body weight. This combination will provide immobilization and sedation, permitting staff to place the facemask over the snout with the animal comfortable for final induction and intubation under isoflurane (2-4\%) inhalational gas. Upon induction of general anesthesia, an endotracheal tube is placed, and the animal is connected to a positive displacement ventilator. An IV catheter is placed in the marginal ear vein of each ear to provide IV access and permit administration of fluids and IV medications as needed. Isoflurane (2-4\%) anesthesia will be maintained and adjusted as needed to ensure full animal anesthesia. EKG leads, pulse oximetry sensor, and rectal temperature probe are placed. We will then ensure the depth of anesthesia by using sensitive interdigital pinch and the palpebral/eyeblink reflex. Also the lack of reflex movement of the animal in response to noxious stimuli during the incisions will indicate the level of anesthesia. The surgical site will be the mid posterior aspect- shoulder (2 devices), mid thoracic pave vertebrae (2 devices), mid lumber pave vertebrae (2 devices), mid sacral (1 device) and they will be scrubbed at least twice with a germicidal scrub, being careful to scrub from the center of the site toward the periphery.

Continued on Next Page 


\section{APPENDIX 2- CONTINUED}

\section{Continued from Previous Page}

Figure1: Picture of $\mathrm{SiC}$ based Ti/Au antenna sensor. The dimension of the device is $(11.5 \mathrm{~mm} \times 8.5$ $\mathrm{mm})$. SiC substrate thickness $-0.373 \mathrm{~mm}$, Ti/Au antenna electrode thickness $-0.45 \mu \mathrm{m}$.

The sites will then be rinsed / wiped with a $70 \%$ alcohol and then painted with dilute, tamed iodine solution. Then the area will be draped with sterile drapes. After preparation, to implant the devices, carefully $1.5 \times 1$ centimete $^{2}$ pockets will be created beneath the skin (dermis) surface. The implants will then be placed into the pocket very carefully using fine tweezers.

Five antennas and two controls, total 7 devices will be implanted into the pockets of a single animal. Each of the five antennas will have different surface coating to evaluate the histological tissue response. The devices are - titanium/gold (Ti/Au) based silicon carbide (SiC) antenna (shown in Fig 1), silicon dioxide $\left(\mathrm{SiO}_{2}\right)$ coated Ti/Au antenna, Poly ethylene glycol (PEG) coated Ti/Au antenna, all SiC antenna, amorphous $\mathrm{SiC}$ coated Ti/Au antenna. The two controls will be silicon (Si) and Parylene $\mathrm{C}$. The five antennas' reactivity to cells/tissue will be compared to these two controls. Biocompatibility and nonbiocompatibility will be verified using the positive reaction control, silicon, and negative reaction control, biocompatible material Parylene C.

After implantation, the deep tissue will be closed with 2-0 suture material polypropylene (Surgilene ${ }^{\circ}$, Prolene ${ }^{8}$. Next the skin will be closed with interrupted non-absorbable suture material, polypropylene (Prolene $^{*}$, Surgilene ${ }^{\bullet}$, Fluorofil ${ }^{\odot}$ ). The same procedure will be repeated for each of the 7 implant devices. The animal will be allowed to recover from the anesthetic regime. Post-operatively, the animals will be monitored continuously and assessed using systemic temperature, pulse rate, respiratory rate, mucous membrane color, and capillary refill time. Then the animals will be recovered in a clean separate place with ancillary heat control. This will continue until the animals are capable of voluntary movement before being transferred to the housing area. The operative procedure will require a time of approximately 15 minutes per implant, for a total of 105 minutes. Following implantation, and recovery from anesthesia, the animal will be housed in a single housing area for 30 days, with routine nourishment, and will be monitored and evaluated every day. A log of observation will be kept for proper monitoring. This single housing is designed to minimize the risk of surgical site trauma resulting from animal interactions. After 30 days, post implant, the animals will again be placed under general anesthesia. Prior to induction of anesthesia, the pig will be fasted for 12 hours. Carprofen will be administered subcutaneously at the dose rate of $4.0 \mathrm{mg} / \mathrm{kg}$. Also prior to induction of general inhalational anesthesia, the animals will receive diazepam (at the initial dose rate of $4.0 \mathrm{mg} / \mathrm{kg}$ body weight $\mathrm{PO}$ ) and atropine sulfate (at the dose rate of $0.05 \mathrm{mg} / \mathrm{kg} \mathrm{IM}$ ). Ketamine will be administered IM at the dose rate of $20 \mathrm{mg} / \mathrm{kg}$ body weight. This combination will provide immobilization and sedation, permitting staff to place the facemask over the snout with the animal comfortable for final induction and intubation under isoflurane (2-4\%) inhalational gas. Upon induction of general anesthesia, an endotracheal tube is placed, and the animal is connected to a positive displacement ventilator. An IV catheter is placed in the marginal ear vein of each ear to provide IV access and permit administration of fluids and IV medications as needed. Isoflurane (2-4\%) anesthesia will be maintained and adjusted as needed to ensure full animal anesthesia. EKG leads, pulse oximetry sensor, and rectal temperature probe are placed. We will then ensure the depth of anesthesia by using sensitive interdigital pinch and the palpebral/eyeblink reflex. Also the lack of reflex movement of the animal in response to noxious stimuli during the incisions will indicate the level of anesthesia. The surgical sites which have been described earlier will be scrubbed at least twice with a germicidal scrub, being careful to scrub from the center of the site toward the periphery. The site will then be rinsed / wiped with a $70 \%$ alcohol and then painted with dilute, tamed iodine solution. Then the area will be draped with sterile drapes. The surgical incisions will be re-opened, and the previously placed implants. The pigs will then be euthanatized using an IV bolus of 1 to $2 \mathrm{mmol} / \mathrm{kg}$ (at least $30 \mathrm{ml}$ ) USP grade of saturated potassium chloride $(\mathrm{KCl})$ overdose while still under general anesthesia. Death will be confirmed by verification of cessation of cardiac and respiratory activity. Immediately after that the surrounding tissue will be removed for analysis. 


\section{APPENDIX 2- CONTINUED}

\section{CHARACTERISTICS OF ANIMALS AND PAIN CATEGORY OF RESEARCH}

10. List and describe the animals to be studied. Indicate the anticipated number of animals to be used in each Pain Category of Research and the total number of animals involved during the 3year approval period of this protocol. Indicate strain or line designation if rodents are requested. Indicate the number of each species/strains that will be involved in planned procedures that are anticipated to produce momentary, slight, or no pain, discomfort or distress (Pain Category A); more than momentary or slight pain, discomfort or distress which is alleviated by the use of appropriate anesthetics and/or analgesics (Pain Category B); or pain, discomfort, or distress, which cannot, or is not alleviated by the administration of appropriate anesthetics and/or analgesics (Pain Category C). (Note: Refer to IACUC Principles \& Procedures XII. The same number of animals requested here must be justified in response to item \#11, below.)

\begin{tabular}{||l|c|l|l|l||}
\hline Species, Strain, or Line & Pig & & & \\
\hline $\begin{array}{l}\text { Characteristics } \\
\text { (age, sex, weight) }\end{array}$ & $\begin{array}{c}\text { Adult } \\
\text { male/female, } \\
20-35 \mathrm{Kg}\end{array}$ & & & \\
\hline $\begin{array}{l}\text { \#used in Pain CategoryA } \\
\text { procedures (no or slight } \\
\text { discomfort). }\end{array}$ & 0 & & & \\
\hline $\begin{array}{l}\text { \#used in Pain Category B } \\
\text { procedures (discomfort } \\
\text { alleviated). }\end{array}$ & 4 & & & \\
\hline $\begin{array}{l}\text { \#used in Pain Category C } \\
\text { procedures } \\
\text { (discomfort not alleviated). }\end{array}$ & 0 & & & \\
\hline Total Number Requested & 4 & & & \\
\hline
\end{tabular}

11. Briefly describe the rationale using statistical analysis, whenever possible, used to determine the total number of each species of animals declared above in response to item \#10 that will be needed for use during the 3-year approval period of this protocol. Section 9 or Section 11 of the application should contain an explanation/rationale for assigning the specific number of animals to the different Pain Categories in Section 10. (Note: For complicated experimental designs, it may be helpful to the IACUC if this explanation indicates the \# of animals needed for each experimental group, the \# of groups required, and the analyses conducted using each group. Alternatively, it may be helpful to the IACUC if a flow chart depicting the sequence of events and the number of animals required for each step is shown.)

The attached table-1 (page 9) details the proposed material/device and experimental groups necessary to reach the conclusions with the current animal request with appropriate statistical comparisons. The total number of animals to be used is an estimate based on how many samples are needed to obtain statistically relevant data for in-vivo/in-vitro experiments. Typically in-vivo/in-vitro studies have a total of 25-30 samples based on literature review. We have selected 4 adult pigs to achieve the statistical relevance for the selected histological tissue/cell evaluation and surface permissiveness to cells because we are planning to implant 5 of the specified different materials/devices (Table 1), one negative control, and one positive control, totaling 7 different materials in each pig. Total 28 (7 different materials $\times 4$ animal number) samples will be implanted for statistically relevance of the results. This study will characterize the surface morphology, permissiveness and evaluate the tissue/cells on the implants as well as surrounding tissue after 30 days implantation using Atomic Force Microscopy (AFM), optical microscopy and Histological tissue analysis to evaluate the in-vivo biocompatibility. For the tests, we will be using bio-compatible material as negative reaction control, parylene $\mathrm{C}$ and a non-biocompatible material as positive reaction control, Silicon just to compare our device/material's in-vivo biocompatibility with them. Because of excellent invivo biocompatibility, parylene $\mathrm{C}$ has little to no cell/tissue attachment. On the other hand after 12-15 weeks (typically) of implantation $\mathrm{Si}$ has modest tissue reaction and fibrous encapsulating tissue on the surface. Therefore, there will be minor pathological responses/no immediate adverse reactions are expected after the implantation of the controls.

Continued on Next Page 


\section{APPENDIX 2- CONTINUED}

\section{Continued from Previous Page}

To maintain animal numbers to a strict minimum, we have selected the materials which have already been proved earlier for in-vitro bio-compatibility. It has been shown by our group previously that $\mathrm{SiC}$ has excellent compatibility with skin and connective tissue cells externally. It has also been shown that the bio-compatibility of covalently bonded Poly ethylene glycol (PEG) which can remarkably reduce the non-specific adsorption of protein. Titanium (Ti), silicon-di-oxide $\left(\mathrm{SiO}_{2}\right)$ and inert material gold (Au) are more or less bio-compatible and frequently used for different implantations.

All animals have been assigned to category $B$ because they will be anesthetized during the procedure.

12. Briefly describe the characteristics of the animals requested that justify its use in this protocol.

The pig will be used in these experiments for some important reasons. The pig model has long been a good tool to help medical researchers to mimic human body size and physiology in order to answer clinical questions. The alternative, the rodent is small in size compared to the human with a different immunologic system, which restricts the usage of this kind of animal model for this study. With the increased use of pig in biomedical research, especially for chronic studies, the availability of early maturing, adult pig has increased. Furthermore our sensor size is not suitable for small animal like the mouse/rat, so the size of the sensor is suitable for implantation in a pig.

\section{IACUC PRINCIPLES AND PROCEDURES CONSIDERATIONS}

13. Will animals be involved in procedures that are anticipated to have the potential to produce more than momentary or slight pain, discomfort, or distress, which cannot, or will not be alleviated by the administration of appropriate anesthetics and/or analgesics?

No: $\mathbf{X}$ Proceed to Item \#14. Yes: $\square$ Within the space below, define the clinical criteria that Will be used to ensure timely intervention and treatment, or removal of the animals from the study, either in advance of, or immediately after recognition of the discomfort. The earliest possible clinical end point, which will contribute to the resolution of the hypothesis, must be identified and utilized. If avoidance or alleviation of animal pain or discomfort adversely affects the protocol, provide a detailed justification of why treatments cannot be initiated.

14. Is animal death (excluding death from euthanasia) an intentional endpoint in this protocol (e.g., survival analysis, radiation, toxicity, or carcinogenesis testing)?

No: $\mathbf{X}$ Proceed to Item \#15. Yes: $\square$ Within the space, explain why an earlier endpoint is norpossible.

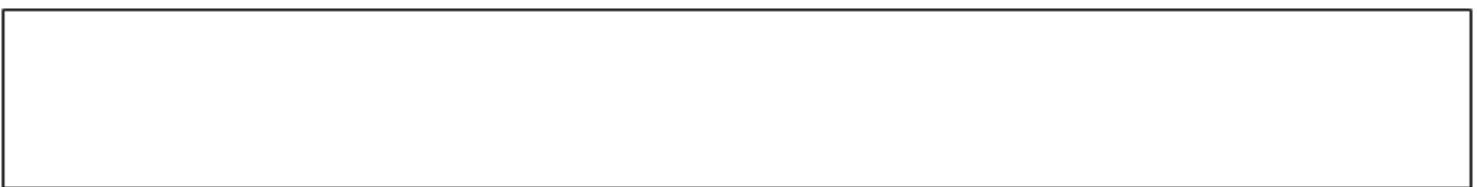

15. Will any animal research/procedural/testing areas outside of the animal facility be used for this protocol?

No: $\mathrm{X}$ Proceed to Item \#16. Within the space, provide a scientific or logistical justification for why animals must be removed from the facility, indicate the location, the 


\section{APPENDIX 2- CONTINUED}

approximate number of hours animals will be held at this site, and whether the animals will need to be returned to housing after the procedure, or that the procedure will be terminal.

W. Will this study be performed in accordance with 21 CFR 58 as a GLP study?

No: $\mathbf{X}$ Proceed to item \#17. Yes: $\square$ Attach a copy of the GLP Study Protocol.

17. Will scheduled substances controlled by the Drug Enforcement Administration be used in the protocol?

No: $\square$ Proceed to item \#18. Yes: $\mathbf{X}$ Within the space, list the controlled substances to

Ketamine, diazepam

\section{EXPERIMENTAL PROCEDURES}

18. Are other than standard and routine husbandry and handling practices required for this protocol (e.g., food, fluid, or caloric restriction, unique diets or nutritional supplements, specialized caging or environments, or non-standard health monitoring)?

No: $\square$ Proceed to Item \#19. Yes: $\mathbf{X}$ Attach "Appendix A: Special Husbandry."

19. Are test substances administered to animals as part of this protocol (e.g., radioisotopes, toxic, immunogenic, pharmacologic, infectious, or carcinogenic agents, biomaterials, or cells)?

No: $\mathbf{X}$ Proceed to Item \#20. Yes: $\square$ Attach "Appendix B: Test Substances."

20. Are specimens collected from animals prior to euthanasia as part of this protocol (e.g., tissues, blood, lymph, or other body fluids)?

No: $\mathbf{X}$ Proceed to Item \#21. Yes $\square$ Attach "Appendix C: Specimen Collection, Ante
Morterm.

21. Will surgery be performed on animals as part of this protocol?

No: $\square$ Proceed to Item \#22. Yes: $\mathbf{X}$ Attach "Appendix D: Surgery."

22. Will animals be subject to experimental procedures other than those described above (e.g., behavioral manipulations, noxious stimuli, forced exercise, or physical restraint)?

No: $\mathbf{x}$ Proceed to Item \#23. $\square$ Attach "Appendix E: Other Experimental Procedures."

23. Will a human patient procedural area be required for this protocol?

No: $\mathbf{X}$ Proceed to item \#24. Yes: $\square$ Attach "Appendix F: Patient Procedural Area." 


\section{APPENDIX 2- CONTINUED}

24. Will Veterans Affairs resources (e.g., VA lab space, VA vivarium, VA equipment, VA funding, VA personnel, VA time) be used in any aspect of this research?

No: $\mathbf{X}$ Proceed to item \#25. Yes: $\square$ Attach "Appendix G: VA Hospital Resources Information."

\section{EUTHANASIA}

25. Are animals euthanatized for postmortem tissue collection, or are euthanatized at the completion of this study?

No: $\square$ Within the space below indicate the final disposition of the involved animals.

Yes: $\mathbf{X}$ Does the method of euthanasia and means of assuring death following euthanasia comply with IACUC

Principles \& Procedures XX which describes acceptable methods of animal euthanasia within these laboratories?

No: $\quad$ Within the space below indicate why a deviation is necessary. Yes: $\mathbf{X}$ Within the space Delow describe the method of euthanasia used for each species requested, indicating dose and route if a chemical agent, or provide a justification if a physical method.

The animal will be euthanized using appropriate agents, at or after 30 days post implantation. The surgical incisions will be reopened and remove the previously placed implants for analysis. Then the animals will be euthanatized for postmortem tissue collection (implants). The final disposition will be entailed deep general anesthesia with Ketamine $(30 \mathrm{mg} / \mathrm{kg})$ and xylazine $(2 \mathrm{mg} / \mathrm{kg}$,). The animals will then be euthanatized using an IV bolus of 1 to $2 \mathrm{mmol} / \mathrm{kg}$ (at least $30 \mathrm{ml}$ ) USP grade of saturated potassium chloride $(\mathrm{KCl})$ overdose while still under general anesthesia. Death will be confirmed by verification of cessation of cardiac and respiratory activity.

STOP here. Complete and attach any required appendixes as indicated above. This application is complete. 


\section{APPENDIX 2- CONTINUED}

\section{APPENDIX A: SPECIAL HUSBANDRY}

(Complete \& Submit for Review Only If Response to Item \#18 was "Yes")

1. Does this protocol involve food, fluid, or caloric restriction? No: $\mathbf{X}$

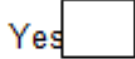

2. Within the space provided, describe all non-standard husbandry and handling practices which are a part of this protocol, including the length of time they will be implemented (e.g., suspended wire mesh caging of rodents, dietary manipulations, food or water deprivation, modified light cycle, specialized housing, confinement, isolation, restricted observation, restricted environmental enrichment, unique requirements of immunocompromised strains).

The pig will be maintained postoperatively in single housing for 30 days

3. Within the space provided, justify the implementation of this requested husbandry practice.

The single housing is designed to minimize the risk of surgical site trauma resulting from animal interactions. This single housing is required and the use of passive/non-interactive monitoring techniques will be maximized as much as possible. Because of this, and to eliminate the stress of scheduled feedings, food will be provided for the post-operative days.

4. Will this special husbandry practice cause more than momentary, slight pain or discomfort to the animals (see policy statement regarding avoiding and alleviating animal pain and discomfort for guidance)?

No: $\mathbf{X}$ Proceed to Item \#5. Yes: $\square$ Within the space below, describe the methods that will be used to minimize pain and discomtor. No: $\mathbf{X}$ Proceed to Item \#5. Yes: $\square$ Within the space below, describe the methods that will
be used to minimize pain and discomfor.

5. Within the space provided, describe the methods for monitoring the condition of the animal during the procedure and during the post-procedural period, and indicate whether a log of observations will be kept.

(Note: Log entries describing procedures involving non-rodent mammals must be kept by the PI in the animal facility on forms provided by Comparative Medicine.)

Since no immediate adverse reactions are expected, animal will be monitored during the routine monitoring schedule of the husbandry personnel. Following implantation, and recovery from anesthesia, the animal will be housed in a single housing area, with routine nourishment, and will be monitored and evaluated every day. A log of observation will be kept for proper monitoring.

Description of Appendix A is complete. 


\title{
APPENDIX 2- CONTINUED
}

\section{APPENDIX D: SURGERY}

\author{
(Complete \& Submit for Review Only If Response to Item \#21 was "Yes")
}

1. Within the space provided, describe the pre-operative medications, including pre-emptive analgesia, tranquilizers, sedatives, pre-anesthetics, and general anesthetics, and their dose and route.

Prior to induction of general inhalational anesthesia, the animals will receive diazepam (at the initial dose rate of $4.0 \mathrm{mg} / \mathrm{kg}$ body weight $\mathrm{PO}$ ) and atropine sulfate (at the dose rate of 0.05 $\mathrm{mg} / \mathrm{kg}$ IM). Ketamine will be administered IM at the dose rate of $20 \mathrm{mg} / \mathrm{kg}$ body weight. This combination will provide immobilization and sedation, permitting staff to place the facemask over the snout with the animal comfortable for final induction and intubation under isoflurane $(2-4 \%)$ inhalational gas.

Upon induction of general anesthesia, an endotracheal tube is placed, and the animal is connected to a positive displacement ventilator. An IV catheter is placed in the marginal ear vein of each ear to provide IV access and permit administration of fluids and IV medications as needed. Isoflurane (2-4\%) anesthesia will be maintained and adjusted as needed to ensure full animal anesthesia. EKG leads, pulse oximetry sensor, and rectal temperature probe are

2. Within the space provided, describe the pre-operative procedures, including the withholding of food or water, (for what period of time), shaving of hair with enough border to prevent contaminating the operative site, two surgical germicidal scrubs of site, draping with sterile drapes, and the placement of leads, probes, or catheters.

\begin{abstract}
Auricular venous catheters, for fluid administration, will be placed in each ear. The site prep will be occurred in an area remote from the surgical area. Hair will be clipped from the surgical site. The surgical site (the back of the pig) will be scrubbed at least twice with a germicidal scrub, being careful to scrub from the center of the site toward the periphery. The site will then be rinsed / wiped with a $70 \%$ alcohol and then painted with dilute, tamed iodine solution. The area will be draped with sterile drapes. This helps prevent stray detritus from entering the suraical field and provides a sterile area on which to lav sterile instruments during surqerv.
\end{abstract}

3. Will paralyzing agents be used?

No: $\mathbf{x}$ Proceed to item \#4. Within the space provided, justify why it is necessary to use them.

4. Within the spaces provided, describe the surgical procedures in chronological order and in sufficient detail so that the IACUC will be able to determine what is being performed on the animal (Note: Intra-operative monitoring, medications and support are to be described under item \#5).

Implantation procedure: After preparation, the pockets will be created $\left(1.5 \times 1\right.$ centimeter $\left.^{2}\right)$ approximately $1 \mathrm{~cm}$ beneath the skin, in the dermis region using electrocautery on the respected surgical locations. The surgical sites will be the mid posterior aspect- shoulder, mid thoracic pave vertebrae, mid lumber pave vertebrae and mid sacral. Then the implants will be placed into the dermis region (pockets) horizontally. After implantation, the deep tissue will be closed with 2-0 suture material polypropylene (Surgilene ${ }^{\oplus}$, Prolene ${ }^{\star}$ ). Next the skin will be closed with interrupted non-absorbable suture material, polypropylene (Prolene ${ }^{\oplus}$, Surgilene ${ }^{\star}$,

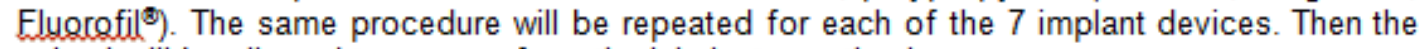
animal will be allowed to recover from the inhalant anesthesia. 


\section{APPENDIX 2- CONTINUED}

5. Within the space provided, describe the methods used for monitoring intra-operative anesthesia (e.g., rate \& depth of respiration, interdigital pinch or palpebral blink reflex), and all intra-operative medications and/or support.

(Note: Log entries describing surgical events involving nonrodent mammals must be kept by the Pl in the animal facility on forms provided by Comparative Medicine. Log entries must at least include a pre-operative assessment, an anestheticplan, records of the induction and monitoring of general anesthesia, a brief description of the surgical procedures performed, a record of the recovery from anesthesia (or method of euthanasia of the anesthetized animal), a post-operative assessment, and any complications, treatments, and/or plans.)

a. Monitoring:

i. Central Venous Access for delivery of fluid and medications

ii. Electrocardiogram Monitoring

iii. Ventilation at $10-15 \mathrm{ml} / \mathrm{kg}$ at $10-15 \mathrm{breath} / \mathrm{min}$

iv. Pulse oximetry (peripheral Oxygen saturation)

v. Capnography (End-tidal Carbon dioxide)

vi. Rectal temperature

vii. Position: Supine with Warming Blanket, Cushioning, and soft restraints

viii. Peripheral venous access will be used for fluid delivery

The sensitive interdigital pinch and the palpebral/eyeblink reflex will be used to monitor depth of anesthesia. Lack of reflex movement of the animal in response to noxious stimuli during the incisions while just under general anesthesia will also indicate that the level of anesthesia is adequate.

6. Will the animal(s) regain consciousness from anesthesia following surgery?

No: $\square$ Stop here, this description is complete. Yes: $\mathbf{X}$ Proceed to item \#7.

7. Indicate below, in which of the established nonrodent mammalian surgical areas will this aseptic surgery be performed, or indicate where surgery will be performed.

COM: $\mathbf{X}$ Other: $\square$ Describe location:

8. Will aseptic techniques be used (refer to IACUC Principles \& Procedures XV), as a minimum including working in an uncluttered area, the wearing of surgical gloves and a clean lab coat, preparation of the surgical site with disinfectant, sterilizing of instruments, and appropriate wound closures)?

No: $\square$ Within the space provided, justify. Yes: $\mathbf{X}$ Proceed to item \#9. 


\section{APPENDIX 2- CONTINUED}

\section{APPENDIX D: SURGERY (cont.)}

9. Animals recovering from general anesthesia must be monitored at least until they are sternal recumbent and capable of purposeful movement. Prior to that point, describe the interval and manner of immediate post-operative monitoring, and clinical reassessment. How frequently will the animals be evaluated, and in what manner will the animal(s) be monitored post-operatively? (Note: Log entries of all post-operative events involving norrodent mammals must be kept by the Pl in the animal facility on forms provided by Comparative Medicine.)

10. Will more than one major surgical procedure be performed on a single animal? (Note: If a survival surgical procedure is followed by a nonsurvival surgical procedure, respond " $\mathrm{No}^{\text {n }}$.)

No: $\mathbf{X}$ Proceed to item \#11. Yes: $\square$ Within the space provided, justify.

11. Within the space provided, describe post-operative patient care after the animals have been returned to long-term housing, including the administration of analgesics, medications, fluids, and any other support methods (dose, route and frequency of post-operative analgesics and medications must be described), and indicate that skin sutures or staples will be removed at approximately 10 - 14 days post-operatively.

The animals will be provided post-operative analgesic agent coverage for the first 24 hours after surgery, then provided analgesic"as needed" (p.r.n.) for the next 48 hours. When used, carprofen would be administered subcutaneously at 12 hour intervals, at the dose rate of $4.0 \mathrm{mg} / \mathrm{kg}$ body weight.

For the thirty (30) days post-operatively, animals will be housed individually and monitored twice daily for any signs of abnormal behavior, including restlessness, listlessness, anorexia, abnormal posture, food intake and fecal output without measurement.

Animals will be kept bandaged for two (2) days to protect the incisions and the dressing removed at the 5 day postopative mark. The incision will be evaluated post-operatively for discharge/drainage, hyperemia (redness), swelling.

If any of these surgical wound site complications become manifest, in addition to seeking veterinary consultation, animals will be treated in accordance with the recommendations of the attending veterinarians.

Pain will be assessed twice daily for 7 days post-operatively. Carprofen will be administered at 12 hour intervals for the first 24 hours after surgery, then provided, as needed, every 12 hours for the next 48 hours. The dose rate for carprofen, given either IM or per os, will be $4.0 \mathrm{mg} / \mathrm{kg}$ body weight.

Description of Appendix D is complete. 


\section{APPENDIX 3: MICROTOMY TISSUE SECTIONING}

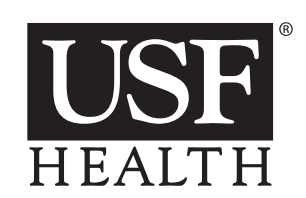

UFS Diagnostic Microscopy Lab

12901 Bruce B. Downs Blvd.

Tampa, FL 33612

\section{Microtomy}

Purpose

Equipment

Special safety precautions

Procedure

sections.

wheel
The purpose of microtomy is to produce thin sections of tissue that can viewed microscopically for diagnostic purposes.

Microtome, disposable blades, forceps, brush, slides, indelible marker, Kimwipes, gauze, and teasing needle.

Microtome blades are extremely sharp and can cause serious injury. Discarded blades should be placed in a biohazard sharps container. Prior to any manipulation of microtome knife or specimen and every time before changing specimens and during breaks, the hand wheel of the microtome must be locked. Never leave knives or knife holders with disposable blades without protection.

1. Trim any excess paraffin from outside of cassette. It is important that the paraffin block fit tight into the microtome chuck for quality

2. Fill water bath with a pinch of gelatin.

3. When water bath has heated to correct temperature, remove bubbles and any debris using a Kimwipe pulled across the surface of the water.

4. Place block in microtome block holder and using the coarse feed to gradually bring the specimen closer to the knife and simultaneously rotate the sectioning wheel. Keep trimming the block until the desired level of the specimen is reached.

5. Return block(s) to ice tray or cold plate. Blocks will section more easily if the blocks are cold and have been allowed to soak. Bloody, decalcified tissue, lymph nodes, and hard fibrous tissue will require longer soaking. 


\section{APPENDIX 3- CONTINUED}

6. Select the desired section thickness with the thickness adjusting knob.

7. For sectioning use another spot of the blade other than the spot used for trimming.

8. Place block in microtome block holder making sure that the block is slightly behind the microtome blade.

9. With a slow, even motion rotate the hand wheel to obtain a ribbon of paraffin sections. 1 revolution per second is considered a reasonable cutting speed.

10. Place the paraffin ribbon on the water bath and select the best sections removing any wrinkles or air bubbles.

11. Separate the sections using a forceps teasing needle or other reliable method.

12. Float the selected section(s) on a glass slide with the same accession number of the block that was sectioned.

13. Allow slides to drain and place in staining rack.

14. Wipe the surface of the water bath with a Kimwipe between each block to prevent floaters between blocks. Poorly processed specimens may explode on the water bath and may make it necessary to change the water in the flotation bath.

15. Continue sectioning until all blocks have been sectioned and a slide(s) has been obtained for each block.

16. Place staining slide rack in $65^{\circ} \mathrm{C}$ drying oven for at least 12 minutes.

17. Sectioning may require multiple levels depending on the type of specimen and any special stains that may be ordered.

18. Multiple levels are required with several types of tissues. The first level is the first complete section. The second level is deeper into the tissue block. The third level is deeper into the tissue block from the second level. When cutting levels always take into consideration of how thick the tissue could be. Valuable tissue could be lost while sectioning levels.

When recuts are requested, sections are taken as soon as possible without going deeper into the block.

19. Levels for colored coded cassettes are as follows:
A. Orange cassette
$\begin{array}{ll}\text { 1. } & 4 \mu \\ 2 . & 1 \text { level }\end{array}$
B Blue cassette
1. $3 \mu$
2. 3 levels
C. Dark green cassette, breast core biopsy
1. $3 \mu$
2. 3 levels
D. Dark green cassette, sentinel lymph node
1. $3 \mu$
2. 2 levels
E. Light green cassette, Medical Examiner's Cases
1. $4 \mu$
2. 1 level 


\section{APPENDIX 3- CONTINUED}

20. Gl biopsies
A. $\quad 3$ levels at $3 \mu$
B. Antrum, duodenum, and gastric biopsies and GI biopsies with a history of or rule out $\mathrm{H}$. Pylori will have a Giemsa stain along with 3 levels.
C. Esophageal biopsies for Barrett's will have 3 levels and AB/PAS stain

21. Sentinel Lymph Nodes
A. 2 levels
B. $3 \mu$

22. Kidney Biopsies
A. $2 \mu$
B. Levels 1-12
C. Deeper panel levels 14-21
D. Levels $1,5,14$, and 18 are for H\&E stain.
E. Place levels 1 and 5 on the same slide.
F. Levels 2, 6, 15, and 19 are for PAS stain.
G. Place levels 2 and 6 on the same slide.
H. Levels 3,11 , and 20 are Trichrome stain.
I. Levels 1,12 , and 21 are for Jones stain.
J. Levels $7,8,13,16$, and 17 are hold slides to be used at a later date by the pathologist.
K. Levels 1-12 are routinely run, but if glomeruli are scarce or more glomeruli are present in slides 14-21 running a deeper panel may be necessary.

23. Research Tissue
A. $\quad 4 \mu$
B. $\quad$ 1-2 sections per slide

\begin{tabular}{|l|c|c|c|c|c|c|c|}
\hline \multicolumn{3}{|c|}{ Routine } & \multicolumn{3}{c|}{ Deepers } \\
\hline H\&E & 1,5 & & 9 & & 14 & & 18 \\
\hline PAS & 2,6 & & 10 & & 15 & & 19 \\
\hline Trichrome & 3 & & 11 & & & & 20 \\
\hline Jones & 4 & & 12 & & & & 21 \\
\hline Hold & & 7,8 & & $13 \mu$ & & 16,17 & \\
\hline
\end{tabular}

\section{Slide examples}




\section{APPENDIX 3- CONTINUED}

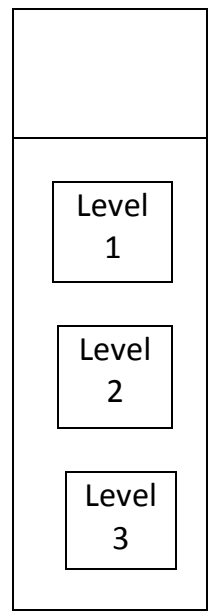

One slide - 3 levels

One slide - 1 level
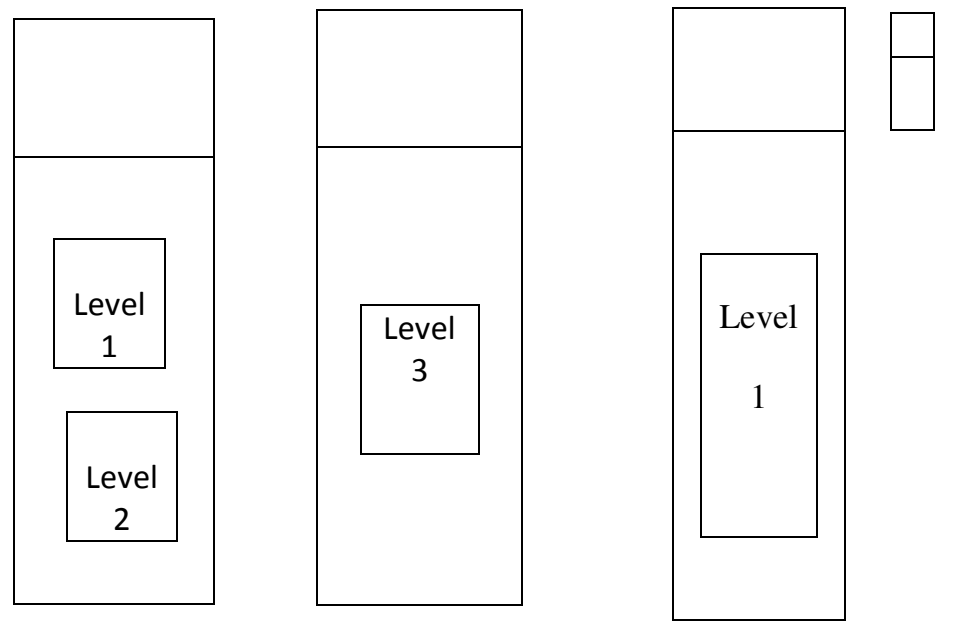

Two slides - 3 levels

Three slides - 2 levels

22. Microtomy Troubleshooting

A. Vertical scratches and splits in sections

1. Hard material such as calcium in block.

2. Defect in knife edge

B. Holes in tissue sections

1. Faced too aggressively

2. Block needs longer soaking

C. Chatter or microscopic vibration in section

1. Caused by over dehydration or dull knife. 


\section{APPENDIX 3- CONTINUED}

2. Soak block longer.

3. Decrease cutting speed.

4. Sharp blade in use, change if necessary.

D. Thick and thin sections

1. Usually occurs with hard fibrous tissues

2. Chuck holder, blade holder, and any other microtome parts are in good working order. Block must not vibrate.

3. Sharp blade in use, change if necessary.

References Carson, Freida L., and Christa Hladik. Histotechnology: A Self-Instructional Text. Chicago: American Society for Clinical Pathology, 2009.

Cambridge Instruments $\mathrm{GmbH}$. Reichert-Jung Instruction Manual for Microtome 2030 Biocut. Heidelberg, Germany: 1988.

Leica Microsystems. Instruction Manual for Leica RM 2125. 1997. 


\section{APPENDIX 4: H\&E STAINING}

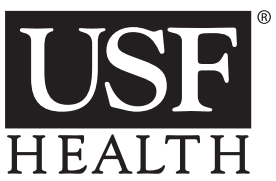

\section{UFS Diagnostic Microscopy Lab 12901 Bruce B. Downs Blvd. \\ Tampa, FL 33612}

H\&E Staining

Purpose

Special safety precautions

Procedure
The purpose of this procedure is define the proper technique so that staining quality can be duplicated with each slide with no variation using the progressive Hematoxylin and Eosin-Phloxine stain.

Chemicals that are used to make the Hematoxylin and EosinPhloxine stain can be irritants, oxidizers, caustic, flammable, and carcinogenic. Take necessary safety precautions when handling these chemicals.

1. After the slides have been dried for at least 12 minutes, remove slides from oven.

2. Place slides in xylene for 3-5 minutes, drain.

3. Place slides in $2^{\text {nd }}$ xylene for 3-5 minutes, drain.

4. Place slides in $100 \%$ alcohol 10 dips, and drain.

5. Place slides in $2^{\text {nd }} 100 \%$ alcohol 10 dips, and drain.

6. Place slides in 95\% alcohol, 10 dips, and drain.

7. Place slides in $2^{\text {nd }} 95 \%$ alcohol, 10 dips, and drain.

8. Place slides in running tap water until slides are completely rinsed of alcohol, and drain.

9. $\quad$ Place slides in Mayer's Hematoxylin for 10 minutes.

10. Place slides in running tap water for 10 minutes.

11. Place slides in 95\% alcohol, 10 dips, and drain.

12. Place slides in Working Eosin-Phloxine stain.

A. Routine surgical slides are stained for 2 minutes in EosinPhloxine stain, and drain.

B. Autopsy and research slides are stained for 5 minutes in Eosin-Phloxine stain, and drain.

13. Place slides in $95 \%$ alcohol, 10 dips, and drain.

14. Place slides in $2^{\text {nd }} 95 \%$ alcohol, 10 dips, and drain. 


\section{APPENDIX 4- CONTINUED}

15. Place slides in $3^{\text {rd }} 95 \%$ alcohol, 10 dips, and drain.

16. Place slides in $100 \%$ alcohol, 10 dips, and drain.

17. Place slides in $2^{\text {nd }} 100 \%$ alcohol, 10 dips, and drain.

18. Place slides in $3^{\text {rd }} 100 \%$ alcohol, 10 dips, and drain.

19. Place slides in xylene, 10 dips, and drain.

20. Place slides in $2^{\text {nd }}$ xylene, 10 dips, and drain.

21. Place slides in $3^{\text {rd }}$ xylene, 10 dips, and drain.

22. Transport slides to fume hood.

23. Remove one slide from rack, place a small amount of mounting media on slide, and coverslip. Place slide onslide board. Continue until all slides are cover slipped and placed on boards for distribution.

24. Results of staining
A. Nuclei... blue with some metachromasia.
B. Cytoplasm......various shades of pink, identifying different tissue components.

25. Hints to achieve good H\&E staining

sure
A.
Check a slide microscopically from each slide basket to be that proper staining as occurred.

B. Do not allow slides to dry at any point during staining.

C. Keep solutions covered when not in use to prevent evaporation and to keep the absolute alcohols from taking up atmospheric moisture. Make sure that solutions completely cover slides.

D. Change complete staining rack weekly. Document on the H\&E QC form.

E. Change and rotate stains if necessary due to increased workload.

F. Do not dehydrate the slides too quickly. Dehydrating solutions also differentiate.

26. When staining frozen sections for $\mathrm{H} \& \mathrm{E}$, do not place slides in xylene. Place slide in $10 \%$ formalin for 5 minutes, rinse in tap water, and then place slides in Hematoxylin. Continue staining with the above procedure.

\section{Mayer's Hematoxylin}

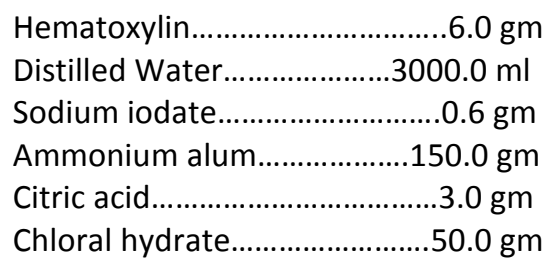

Dissolve the alum in water, without heat, and dissolve the Hematoxylin in this solution. Then add the sodium iodate, citric acid, and the chloral hydrate until all components are in complete solution. The final color of the stain is violet. Stain keeps well for months. 


\section{APPENDIX 4- CONTINUED}

\section{Eosin-Phloxine Solution}

\section{Stock Eosin}

Eosin Y, water soluble. $30.0 \mathrm{gm}$

Distilled water. $3000.0 \mathrm{ml}$

\begin{tabular}{|c|}
\hline Stock Phloxine \\
\hline 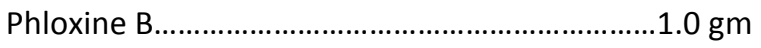 \\
\hline 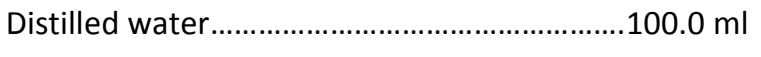 \\
\hline Working Solution \\
\hline 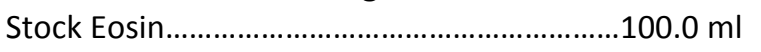 \\
\hline 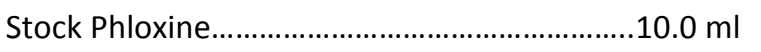 \\
\hline Alcohol, 95\%.......................................... $780.0 \mathrm{ml}$ \\
\hline Glacial acetic acid................................ \\
\hline
\end{tabular}

Make up working solution as needed. Working solution should be changed at least once weekly.

Below is a list of the chemicals used in H\&E staining and type of hazard of each of these chemicals. See MSDS

\begin{tabular}{|c|c|}
\hline Chemical & Hazard \\
\hline Hematoxylin & IRRITANT \\
\hline Sodium lodate & IRRITANT, OXIDIZER \\
\hline Ammonium Alum & IRRITANT \\
\hline Citric Acid & CAUSTIC \\
\hline Chloral hydrate & IRRITANT \\
\hline Eosin & CARCINOGEN \\
\hline Phloxine B & CARCINOGEN \\
\hline Stock Eosin & CARCINOGEN \\
\hline Stock Phloxine & FLAMMABLE \\
\hline 95\% Alcohol & CAUSTIC \\
\hline Glacial Acetic Acid & FLAMMABLE, USE IN WELL \\
\hline Xylene & VENTILATED AREA. PROLONGED \\
& EXPOSURE TO FUMES OR \\
& CONTACT WITH SKIN MAY CAUSE \\
& SERIOUS HEALTH HAZARD. \\
\hline Eosin-Phloxine & FLAMMABLE \\
\hline Mayer's Hematoxylin & IRRITANT \\
\hline & FLAMMABLE, POSSIBLE \\
& CARCINOGEN \\
\hline
\end{tabular}

References

Carson, Freida L. and Christa Hladik. Histotechnology: a Self-Instructional Text. Chicago: American Society of Clinical Pathology. 2009.

Luna, Lee G. Manual of Histologic Staining Methods of the Armed Forces Institute of Pathology. New York: Blakiston Division, McGraw-Hill. 1968. 